\title{
Regioselective Diversification of 2,1-Borazaronaphthalenes: Unlocking Isosteric Space via C-H Activation
}

\author{
Geraint H. M. Davies, ${ }^{\dagger, \| \odot ~ M a t t h i e u ~ J o u f f r o y, ~}{ }^{\dagger, \|}$ Fatemeh Sherafat, ${ }^{\dagger, \dagger}$ Borna Saeednia, ${ }^{\dagger, \S(0)}$ \\ Casey Howshall, ${ }^{\dagger}$ and Gary A. Molander*, ${ }^{\dagger}$ (0)
} ${ }^{\dagger}$ Roy and Diana Vagelos Laboratories, Department of Chemistry, University of Pennsylvania, 231 South 34th Street, Philadelphia,
Pennsylvania 19104-6323, United States
${ }^{\ddagger}$ School of Chemistry, College of Science, University of Tehran, PO Box 141556455 Tehran, Iran
${ }^{\S}$ Laboratory of Organic Synthesis and Natural Products, Department of Chemistry, Sharif University of Technology, Azadi Street, PO
Box 111559516 Tehran, Iran

\section{Supporting Information}

ABSTRACT: Methods for the regioselective $\mathrm{C}-\mathrm{H}$ borylation and subsequent cross-coupling of the 2,1-borazaronaphthalene core are reported. Azaborines are dependent on $\mathrm{B}-\mathrm{N} / \mathrm{C}=\mathrm{C}$ isosterism when employed in strategies for developing diverse heterocyclic scaffolds. Although 2,1-borazaronaphthalene is closely related to naphthalene in terms of structure, the argument is made that the former has electronic similarities to indole. Based on that premise, iridium-mediated $\mathrm{C}-\mathrm{H}$

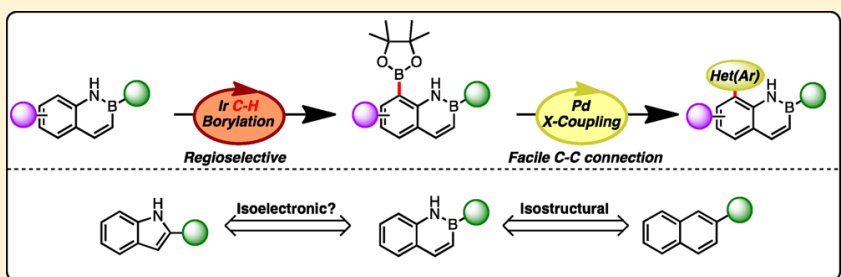
activation has enabled facile installation of a versatile, nucleophilic coupling handle at a previously inaccessible site of 2,1borazaronaphthalenes. A variety of substituted 2,1-borazaronaphthalene cores can be successfully borylated and further crosscoupled in a facile manner to yield diverse C(8)-substituted 2,1-borazaronaphthalenes.

\section{INTRODUCTION}

Isosteres provide versatile molecular scaffolds to optimize chemical space for biological and materials applications. ${ }^{1}$ By providing a diverse array of exchangeable, electronically and sterically related chemical motifs, isosteres allow molecules to be fine-tuned for specific needs. Azaborines exemplify one such class of valuable compounds. ${ }^{2}$ Capitalizing on the ability of the $\mathrm{B}-\mathrm{N}$ bond to mimic a $\mathrm{C}=\mathrm{C}$ bond, numerous aromatic and heteroaromatic systems have been successfully imitated with prospects for rapid diversification patterns not available in the parent system. The 2,1-borazaronaphthalene core is one of the most prominent azaborine systems, highlighted by robust synthetic methods and several strategies for derivatization. ${ }^{3}$ Furthermore, the potential bioisosteric viability of this specific system has been recently demonstrated in comparisons with a $\beta$-blocker ${ }^{4 \mathrm{a}}$ and phosphodiesterase $10 \mathrm{~A}$ inhibitors. ${ }^{4 \mathrm{~b}}$

The versatility of 2,1-borazaronaphthalenes arises from the desymmetrization of the heterocyclic core, providing several modes of functionalization (Figure 1). Our group reported accessing these substructures from $o$-aminostyrene and trifluoroborate derivatives, providing diverse substituents off both boron and nitrogen. ${ }^{3 a}$ Additionally, diversification off boron can be established by nucleophilic substitution and crosscoupling strategies. ${ }^{3 c, d}$ Based on the electronics of the system, selective bromination occurs at the $\mathrm{C}(3)$-position, followed by secondary bromination at $\mathrm{C}(6)$. Recently, halogenation at $\mathrm{C}(4)$ has been reported when accessing the 2,1-borazaronaphthalene from the corresponding 2-alkynyl-anilines with dichlorobor-

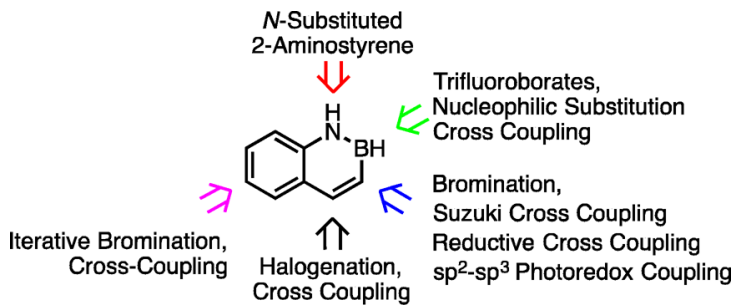

Figure 1. Diversification of the 2,1-borazaronaphthalene core.

anes. $^{3 g}$ The brominated cores can be functionalized by traditional cross-coupling, ${ }^{3 \mathrm{~b}, \mathrm{~g}}$ reductive-coupling, ${ }^{3 \mathrm{e}}$ and even $\mathrm{sp}^{2}-\mathrm{sp}^{3}$ photoredox cross-coupling, ${ }^{3 \mathrm{f}}$ to install myriad functional groups. Employing an iterative process, this protocol has been successfully applied to the sequential 3,6-difunctionalization of 2,1-borazaronaphthalene cores. ${ }^{3 b}$ However, access to the remaining positions of substitution has continued to be challenging, limiting the viability of the system as a whole. This is exemplified in the synthesis of 2,1-borazaronaphthalene mimetics of propranolol, ${ }^{4 a}$ where $C(5)$ and $C(8)$ ethereally substituted 2,1-borazaronaphthalene derivatives required preinstallation of the oxygen functional unit, followed by protection, deprotection, and two subsequent modification steps to introduce the desired side chain. Although such a synthesis might be tolerated from an exploratory, chemical

Received: May 30, 2017

Published: July 17, 2017 
design viewpoint, this strategy would be inefficient for a more expansive molecular diversity study, costing significant time, effort, and material.

Reaction Design and Optimization. When considering ways to expand the 2,1-borazaronaphthalene motif, installing a nucleophilic coupling handle directly on the azaborine core would be beneficial. This would present an alternative approach to those employing an electrophilic, brominated core, opening up a wide array of complementary transformations. ${ }^{5}$ One major drawback to most classical methods for installing nucleophilic coupling partners is that they are often derived from the corresponding halides, effectively passing through the electrophilic coupling partner in an inefficient synthetic pathway. A direct $\mathrm{C}-\mathrm{H}$ borylation would circumvent that limitation and would lead directly to a readily diversifiable handle on the azaborine core. ${ }^{6}$ Inspired by the report of the borylation of 1,2dihydro-1,2-azaborine, ${ }^{7}$ along with the diversity of methods for bicyclic systems, it was envisioned that iridium-catalyzed $\mathrm{C}-\mathrm{H}$ borylation would provide a versatile platform capable of high regioselectivity.

Structurally, the 2,1-borazaronaphthalene is isosteric to naphthalene, which diborylates as a mixture of 2,6- or 2,7disubstituted isomers (Figure 2A), ${ }^{8}$ owing to a lack of inherent

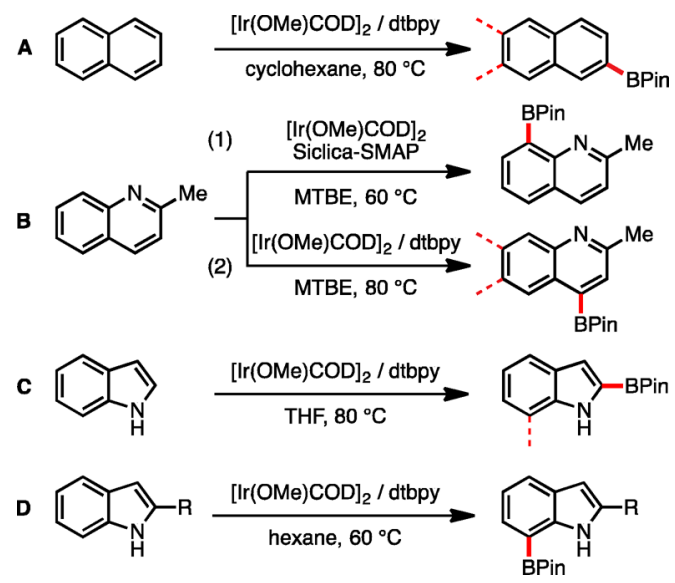

Figure 2. Iridium-catalyzed $\mathrm{C}-\mathrm{H}$ borylation of bicyclic systems. The first site of borylation depicted in bold; second sites of borylation are depicted depicted by dotted line.

directing effects. Quinolines, which have a similar atomic placement, undergo $\mathrm{C}(8)$-borylation using silica-supported phosphine ligands (Figure 2B1). ${ }^{9}$ With traditional amine ligands, borylation of quinoline is predominantly favored at the $\mathrm{C}(4)$-position (Figure 2B2), with subsequent addition at either the $C(6)$ - or $C(7)$-positions. ${ }^{10}$ Indoles, which possess an aromatic $\mathrm{N}-\mathrm{H}$ functional unit with a geometric trade-off to a 6,5-ring system, typically borylate at the $\mathrm{C}(2)$-position. ${ }^{11}$ However, introducing substitution at the $\mathrm{C}(2)$-position of indole, which is consonant with $B$-substitution on 2,1borazaronaphthalenes, causes exclusive borylation at the $\mathrm{C}(7)$-position (Figure 2D). ${ }^{12}$ Furthermore, it is interesting to note the similarity between the electrophilic bromination patterns of 2-phenylindole and 2-phenyl-2,1-borazaronaphthalene, as both brominate at the 3-position (Figure 3). ${ }^{3 \mathrm{~b}, 13}$

Considering these three representative bicyclic models for 2,1-borazaronaphthalene, computationally derived electrostatic potential maps were compared to determine their surface electronic similarities (Figure 4). Cursory observation validates

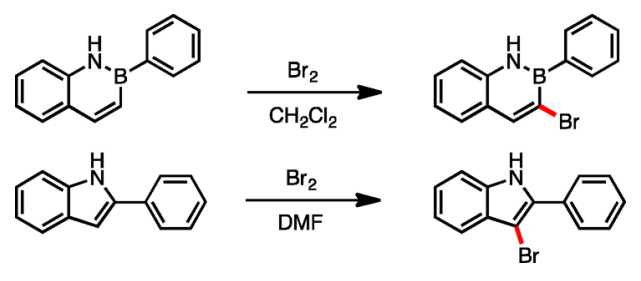

Figure 3. Electrophilic bromination of 2-phenyl 2,1-borazaronaphthalene and 2-phenylindole.
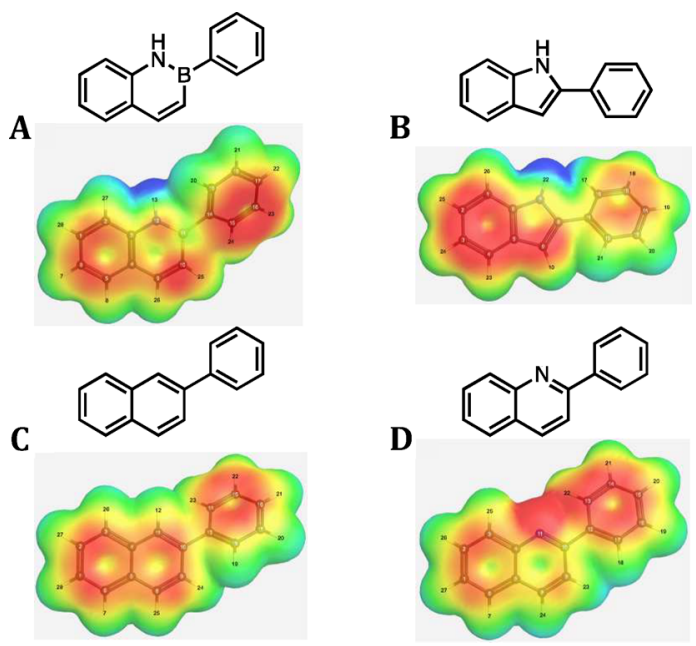

Figure 4. Electrostatic potentials (from $+31.38 \mathrm{kcal} / \mathrm{mol}$ to -15.69 $\mathrm{kcal} / \mathrm{mol}$ ) for bicyclic systems. Calculations performed at B3LYP/6$31 \mathrm{G}(\mathrm{d})$ level of theory ${ }^{14}$ using Gaussian $09^{15}$ visualized via WebMO. ${ }^{16}$

the archetypal resemblance of the 2-phenyl-2,1-borazaronaphthalene (Figure 4A) to 2-phenylnaphthalene (Figure 4C). However, with our interest in leveraging the desymmetrization of the azaborine core, a deeper look into the distribution of electron density across the surface of the molecules yields a clear likeness to 2-phenylindole (Figure 4B). In both the azaborine and indole electrostatic potential maps, a significant density of electrons resides around the $\mathrm{N}-\mathrm{H}$ bond, not seen in either of the other structures (Figure 4C,D). Also, there appears to be a distinct similarity in the asymmetric distribution of the partially electron-deficient region within the bicyclic cores. Encouraged by the congruence of the electrostatic potentials and the comparable reactivity profile for electrophilic bromination, indole could be considered electronically comparable and potentially useful in providing a foundation to predict the selectivity of $\mathrm{C}-\mathrm{H}$ borylation.

The iridium-catalyzed borylation of 2-substituted indoles and similarly structured aniline scaffolds have been extensively studied by Smith and co-workers, with the anilines proposed to go through a mechanism in which outer-sphere hydrogen-bond coordination of the nitrogen occurs to direct the $\mathrm{C}-\mathrm{H}$ functionalization. ${ }^{12,17}$ The nitrogen of indole is suggested to be incorporated in the mechanism, providing selectivity for the $\mathrm{C}(7)$-position. Computational elucidation of the iridiumcatalyzed $\mathrm{C}-\mathrm{H}$ borylation of several aromatic systems indicates that the transition state for $\mathrm{C}-\mathrm{H}$ activation, which is predictive of the regioselectivity, contains "significant proton-transfer character" ${ }^{18}$ It was surmised that the relative anionic stability of the different $\mathrm{C}-\mathrm{H}$ centers of the 2,1-borazaronaphthalene core, a trait of proton-transfer processes, could generally predict the site of $\mathrm{C}-\mathrm{H}$ borylation in these systems. This method is 
analogous to Liu's use of theoretically derived $\mathrm{p} K_{\mathrm{a}}$ values in justifying the site selectivity for iridium $\mathrm{C}-\mathrm{H}$ borylation of 1,2 dihydro-1,2-azaborine.?

To validate this approach, computational comparisons of the anion stability at various positions around 2-phenylindole were used as a representative system (see Supporting Information for complete anionic stability studies). Surprisingly, both the C(3)position and the ortho-site of the external phenyl ring were more anionically stabilizing than the known $\mathrm{C}(7)$-borylation site (Figure 5). However, it is well documented that those sites
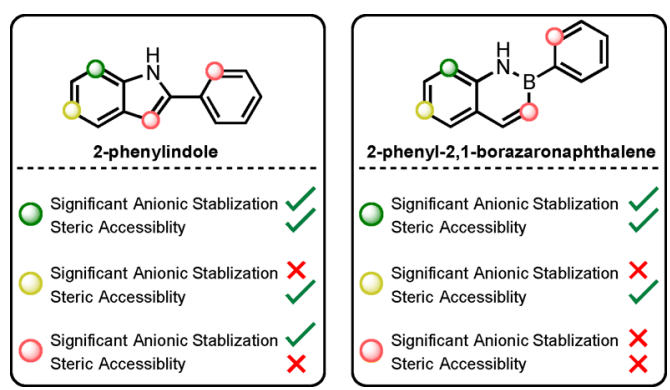

Figure 5. Regioselective analysis for iridium $\mathrm{C}-\mathrm{H}$ activation for 2phenyl indole and 2-phenyl-2,1-borazaraonaphthalene.

are inaccessible based on steric factors, considering the steric bulk of the iridium catalyst. ${ }^{19}$ Anionic stability only accounts for the electronic factors of the $\mathrm{C}-\mathrm{H}$ activation event, thus steric constraints must also be entertained. Removing these two nonviable sites, the theoretical method correctly predicted the C(7)-site for borylation. Applying this method to 2-phenyl-2,1borazaronaphthalene, the $\mathrm{C}(8)$-position appeared to be significantly more amenable to anionic charge stabilization that any other position. Considering this predicted site of borylation in the azaborine has no foreseeable steric constraints, this functionalization method was anticipated to provide access to a previously unattainable position of the 2,1-borazaronaphthalene.

Excited by the potential regioselectivity of the $\mathrm{C}-\mathrm{H}$ activation strategy, high-throughput experimentation (HTE) was leveraged to survey the various reported $\mathrm{C}-\mathrm{H}$ borylation conditions for optimal regioselectivity for the 2,1-borazaronaphthalene system (eq 1 ). ${ }^{6 f}$ In total, close to 450 conditions

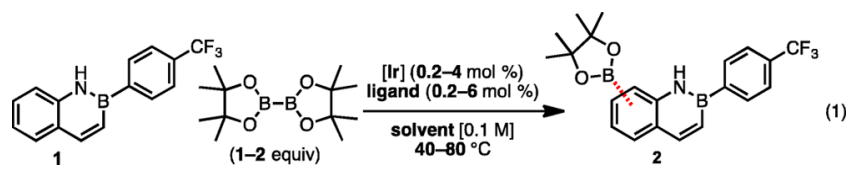
(1)

(see Supporting Information) were screened to assess variables such as iridium sources, ligands, solvents, and relative ratios of these components. Bipyridine- and phenanthroline-based ligands proved to be superior to other nitrogen-containing homobidentate ligands, with reaction incorporating those ligands going to full conversion. In contrast, diphosphine ligands showed minimal conversion. ${ }^{6 \mathrm{~g}}$ It was further found that catalyst loading was optimal at $4 \mathrm{~mol} \%$ with a 1:1.25 metal-toligand ratio. A slight excess (1.05 equiv) of $\mathrm{B}_{2} \mathrm{Pin}_{2}$ was required for the reaction to go to completion. However, significant excess led to an increase of diborylated product. As previously observed, solvent played an important role in controlling selectivity, with nonpolar solvents such as hexane and methylcyclohexane proving optimal.
Upon validation of these conditions on benchtop scale, it was observed that higher reaction concentration coincided with increased diborylation. Temperature also played a significant role in sequestering an $\mathrm{N}$-borylated byproduct and preventing significant diborylation. However, given that the solubility of some of the substrates in methylcyclohexane was very low at room temperature, those less soluble substrates required elevated temperatures $\left(40{ }^{\circ} \mathrm{C}\right.$ vs $\left.80{ }^{\circ} \mathrm{C}\right)$ for the reaction to proceed. NMR analysis of the isolated, monoborylated compound indicated exclusive borylation at the previously predicted (C)8-position, and this was later confirmed via single crystal X-ray diffraction analysis (Figure 6).

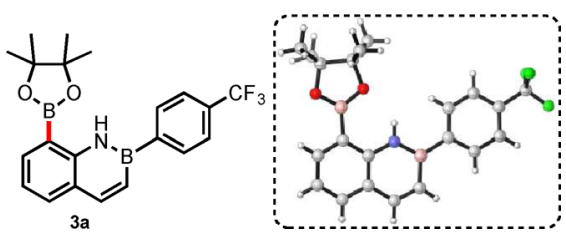

Figure 6. X-ray crystal structure of C(8)-borylated 2,1-borazaronaphthalene.

C-H Borylation Substrate Scope. With conditions for $\mathrm{C}-\mathrm{H}$ borylation at the (C)8-position of 2,1-borazaronaphthalene established, the applicability to various substrates was considered, starting with substitution off of the boron of the borazine (Table 1). A variety of different $B$-aryl-2,1borazaronaphthalene substructures $(3 \mathbf{a}-\mathbf{k})$ were tolerated with various levels of success. Electronic diversification off the aryl substituent seemed to have little impact on the reaction outcome (compare 3a,d,h). However, the substitution pattern off the $B$-aryl subunit did influence the effectiveness of the reaction, with para-substitution being preferred over either meta- or ortho-substitution (compare $3 \mathbf{e}$ to $3 \mathbf{f}$ and $\mathbf{3 h}-\mathbf{j}$ ). Furan substitution $(\mathbf{3 m})$ was tolerated, albeit modestly, as it presents a competing site for $\mathrm{C}-\mathrm{H}$ borylation. ${ }^{20}$ Alkyl-substituted 2,1borazaronaphthalenes $(3 \mathbf{3 o}-\mathbf{r})$ were typically well behaved, presumably because of the increased solubility in the reaction solvent. In almost all cases, the mass balance for the reaction consisted exclusively of starting material, product, and various amounts of a single diborylated byproduct, suggesting that reoptimization for a specific substrate would provide a high return of product.

More elaborate 2,1-borazaronaphthalenes were next explored and found to be competent in the selective $\mathrm{C}-\mathrm{H}$ borylation process (Table 2). 3-Bromo-2,1-borazaronaphthalenes (4a,b) and the 3,6-dibrominated core (4c) reacted reasonably efficiently, as did a substrate with aryl substitution at the 3position (4d), a cross-coupled product of $4 a^{3 b}$ 7-Fluoro-2,1borazaronaphthalene was also suitable in the selective reaction, providing $4 \mathbf{e}$ in modest yield. However, no other substituent at the 7-positions (e.g., i-Pr, Me) allowed productive borylation. Presumably, the steric constraints imparted by substitution at this position prevent accessibility by the sterically congested catalyst. Functionalization at the 6-position was well tolerated, including substrates containing cyano, (4f), trifluoromethyl $(4 \mathrm{~g})$, isopropyl (4h), and trifluoromethoxy (4i) groups. Compounds with electrophilic coupling handles, either installed off the boron subunit $(3 \mathbf{g}-\mathbf{k})$ or the brominated borazaronaphthalene core $(\mathbf{4 a}-\mathbf{c})$, provide potential lynchpins for rapid core diversification. One such avenue could be photoredox cross-coupling, which has demonstrated tolerance of aryl 
Table 1. Borylation Scope of Boron-Substituted 2,1-Borazaronaphthalenes

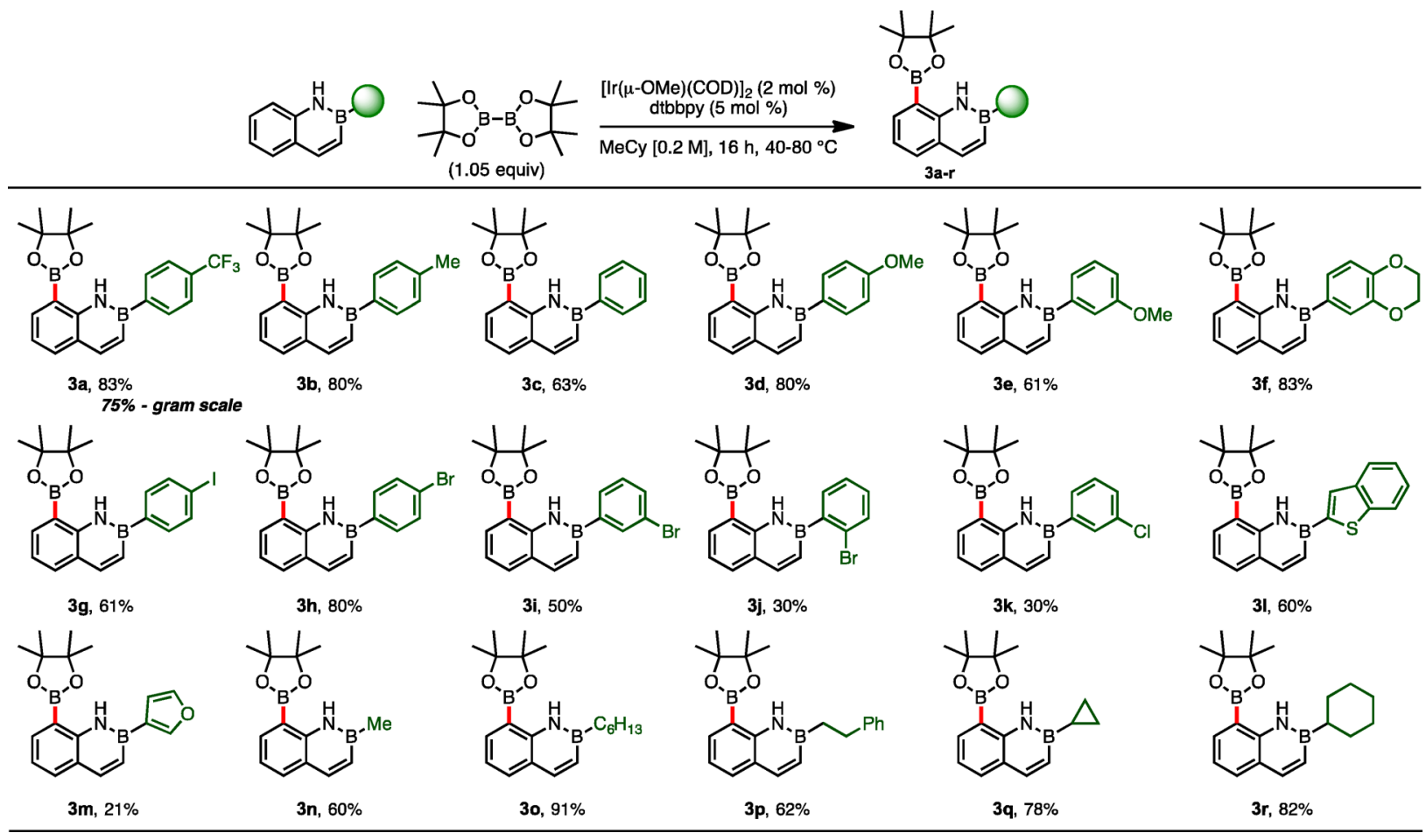

Table 2. Borylation of Elaborated 2,1-Borazaronaphthtalene Scaffolds

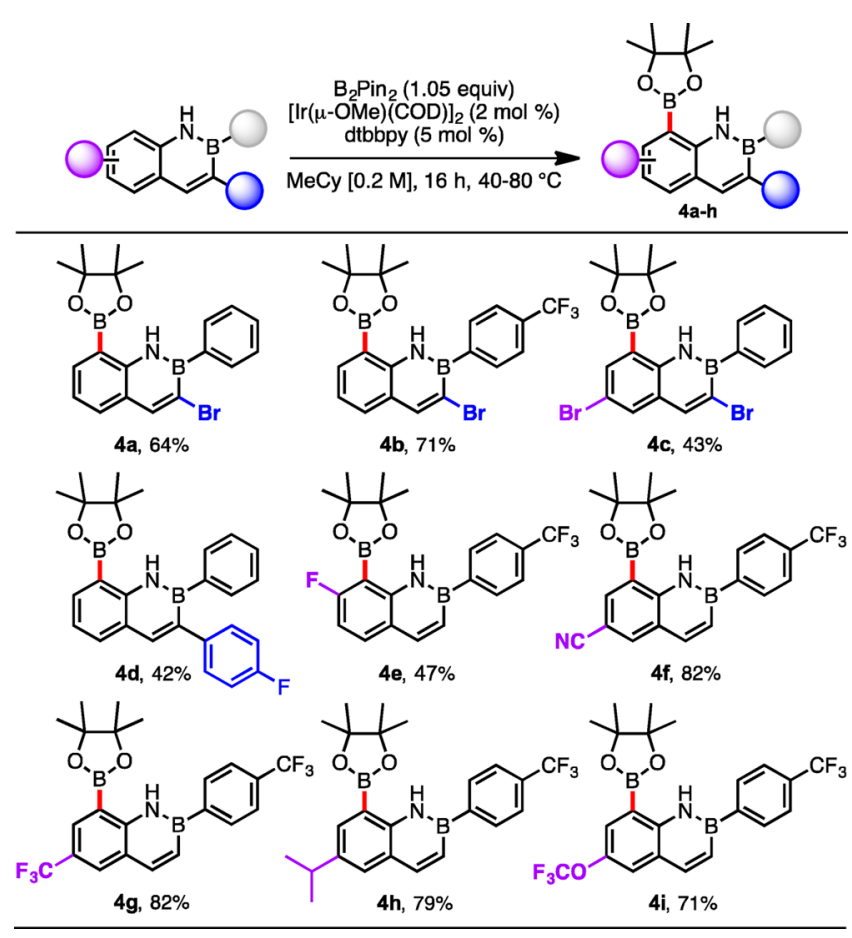

borinates ${ }^{21}$ and has already been applied with great success to azaborines. ${ }^{3 \mathrm{f}}$

As previously mentioned, the exclusive byproduct affecting yields came from diborylation. Fortuitously, the second $\mathrm{C}-\mathrm{H}$ borylation event again occurrs regioselectively, this time at the $\mathrm{C}(6)$-position (Figure 7). When increasing the amount of $\mathrm{B}_{2} \mathrm{Pin}_{2}$ in the reaction, this product can be purposely formed (Table 3). These diborylated cores $(\mathbf{5 a}-\mathbf{c})$ might be further

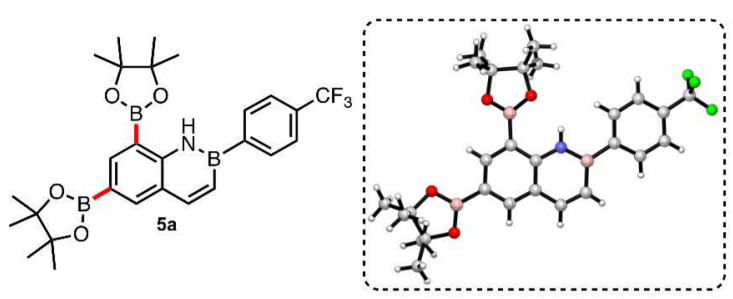

Figure 7. X-ray validation of 6,8-diborylated 2,1-borazaronaphthalene.

Table 3. Diborylation of 2,1-Borazaronaphthalene Cores

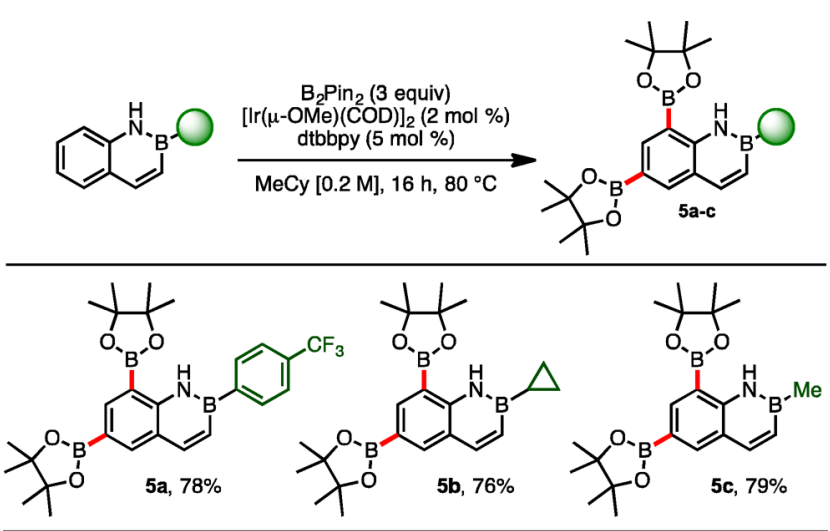

elaborated in divergent functionalization, capitalizing on the stereoelectronic disparities between the sites of borylation, similar to prior work of selective cross-coupling of polyborylated molecules. 22

While screening the borylation of the 2,1-borazaronaphthalenes, one observed phenomenon was the formation of the $\mathrm{N}$-borylation product. Although only observed in trace amounts at elevated temperatures and lower ligand to metal ratio, similar products have been previously noted and used as an in situ 
"traceless" directing group for the $\mathrm{C}(3)$ borylation of indoles. ${ }^{17 \mathrm{a}}$ To probe whether this byproduct was an intermediate of the reaction pathway, the $\mathrm{N}$-deuterated 2,1-borazaronaphthalene 6 was synthesized and subjected to the borylation conditions (eq 2 ). No scrambling of the deuterium was observed in the crude
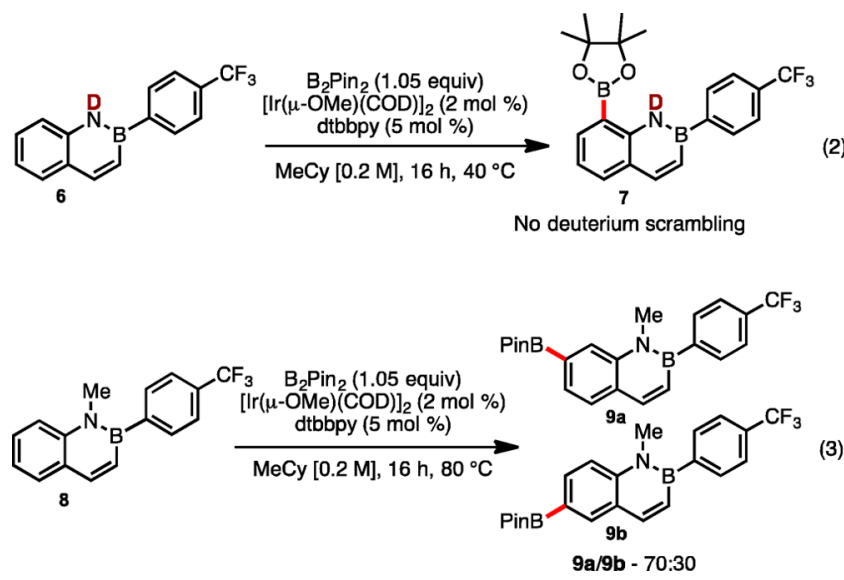

reaction mixture. The $\mathrm{C}(8)$-borylated, $\mathrm{N}$-deuterated product 7 could be isolated, suggesting that the $\mathrm{N}-\mathrm{H}$ insertion, required to obtain the N-borylated product, is not part of the mechanistic pathway for borylation at the $\mathrm{C}(8)$-position. However, it does appear that the $\mathrm{N}-\mathrm{H}$ is intrinsically valuable for the regiospecificity of the borylation. When the $N$-methyl2,1-borazaronaphthalene $\mathbf{8}$ was subjected to the borylation reaction, complete conversion was observed to an inseparable mixture of two regioisomeric products (eq 3). Analysis by 2D NMR suggests that none of the $\mathrm{C}(8)$-borylated product was obtained. Rather, the isomers obtained were the $\mathrm{C}(7)$-borylated 9a and C(6)-borylated 9b 2,1-borazaronaphthalenes, with the major product being the $\mathrm{C}(7)$-borylated isomer. The relative ratio of observed products concurs with the computational model as the $\mathrm{C}(7)$-position has greater favorable anionic charge stabilization compared to the $\mathrm{C}(6)$-positions. It is interesting to note that while $\mathrm{C}(7)$ is the second most favorable point of anionic stabilization, $\mathrm{C}(6)$ ranks fifth, behind both $\mathrm{C}(5)$ and $\mathrm{C}(4)$. The lack of observed borylation at those sites could be explained by a peri-interaction, ${ }^{23}$ marginally disfavoring those sites. This further suggests that the $\mathrm{N}-\mathrm{H}$ could play a fundamental role in enabling the $\mathrm{C}(8)$-borylation event, consistent with the outer-sphere, hydrogen-bond coordination model used to explain the ortho- $\mathrm{C}-\mathrm{H}$ borylation for similarly structured anilines. ${ }^{17 \mathrm{~b}}$

Cross-Coupling of 8-Borylated 2,1-Borazaronaphthalenes. With a diverse array of borylated compounds in hand, the viability of the subsequent Suzuki-Miyaura cross-coupling reaction was studied. Taking from conditions previously established for the inverted coupling of brominated 2,1borazaronaphthalene with organotrifluoborates, ${ }^{3 b}$ with only minor modification to the solvent and ligand choice, the reaction worked exceedingly well. It was observed that alcoholic solvent mixtures with water were highly effective (Table 4) and that sterically bulky alcohols produced the best results (entries 3 and 4), avoiding proto-deborylation.

These optimized conditions were then tested on a variety of the borylated 2,1-borazaronaphthalene cores, focusing on adding heterocyclic diversity to a variety of substituted azaborine substrates (Table 5). Across the board, the yields were generally excellent, with several of the reactions
Table 4. Solvent Optimization of Cross-Coupling ${ }^{a}$

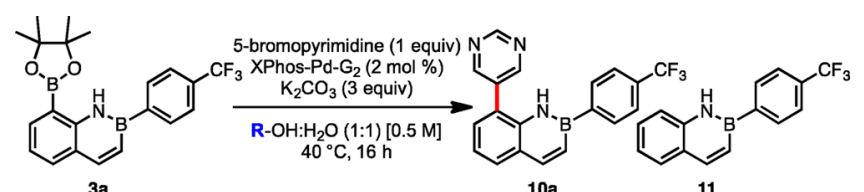

3a

$10 a$

11

\begin{tabular}{l|crrr} 
& Alcohol & 3a : IS & 10a : IS & $\mathbf{1 1}:$ IS \\
\hline $\mathbf{1}$ & MeOH & 0.07 & 1.93 & 0.02 \\
$\mathbf{2}$ & EtOH & 0.08 & 2.18 & 0.18 \\
$\mathbf{3}$ & i-PrOH & 0.07 & 2.54 & 0 \\
$\mathbf{4}$ & $t-\mathrm{BuOH}$ & 0.03 & 2.62 & 0 \\
$\mathbf{5}$ & phenol & 0.00 & 0.30 & 1.40 \\
$\mathbf{6}$ & cyclohexanol & 0.00 & 1.63 & 1.53
\end{tabular}

${ }^{a}$ Product ratios determined by HPLC using 4,4'-di-tert-butylbiphenyl as internal standard (IS).

proceeding nearly quantitatively $(\mathbf{1 0 a}, \mathbf{c}-\mathbf{e}, \mathbf{h}, \mathbf{i}, \mathbf{o})$. Indeed, for reactions that went to full conversion, oftentimes the only purification required was simple aqueous workup followed by rapid purification through a plug of silica to remove the palladium catalyst. In most cases, yield losses corresponded to the required use of column chromatography, owing to incomplete reaction. 5-Bromopyrimidine cross-coupled well with various 8-borylated 2,1-borazaronaphthalene analogues $(10 \mathrm{a}-\mathrm{c})$. Pyridine $(\mathbf{1 0 d})$, quinoline $(10 \mathrm{e})$, and thiophene $(\mathbf{1 0 f}, \mathrm{g})$ substrates also worked well. Although highly functionalized cores such as caffeine could easily be installed with little difficulty $(\mathbf{1 0 h}, \mathbf{i})$, substitution on the all-carbon ring of the azaborine core seemed to hinder the reaction $(\mathbf{1 0 j}, \mathbf{k})$. Standard aryl bromides could also be cross-coupled in high yields (10nq), including challenging electron-rich $N, N$-dimethylamino derivatives $(10 \mathrm{p}, \mathbf{q})$. Unusual substuctures were synthesized using both nucleophilic and electrophilic 2,1-borazaronaphthalene derivatives that could be conjoined to generate heterodimeric-azaborines $(\mathbf{1 0 1}, \mathbf{m})$.

\section{CONCLUSIONS}

In summary, a protocol for the selective $\mathrm{C}-\mathrm{H}$ borylation and subsequent cross-coupling of 2,1-borazaronaphthalenes has been developed, allowing late-stage functionalization of a previously inaccessible position (Figure 8). Furthermore, an isoelectronic correlation of 2,1-borazaronaphthalene to indole is presented for the first time. Computational methods to account for the electronic factors of proton-transfer-like $\mathrm{C}-\mathrm{H}$ activation were applied to identify the potential site of borylation. High-throughput experimentation was utilized for rapid identification of viable reaction conditions that were applicable across a wide array of substrates. Subjecting the reaction to excess $\mathrm{B}_{2} \mathrm{Pin}_{2}$ resulted in the exclusive formation of a diborylated adduct, providing an unprecedented opportunity for chemoselective functionalization in the all-carbon ring. Furthermore, it was demonstrated that the $\mathrm{N}-\mathrm{H}$ is imperative for selectivity, although the reaction is most probably not going through a productive $\mathrm{N}-\mathrm{H}$ insertion pathway. The resulting borylated substrates could be further cross-coupled using mild Suzuki-Miyaura conditions to provide highly elaborated azaborine scaffolds. These developed methods open up new opportunities for the diversification of 2,1-borazaronaphthalene and thus entries into unexplored isosteric space wholly inaccessible to the all-carbon naphthalene core itself. 
Table 5. Cross-Coupling of (Hetero)aryl Bromides with 8-Borylated 2,1-Borazaronaphthalene Cores
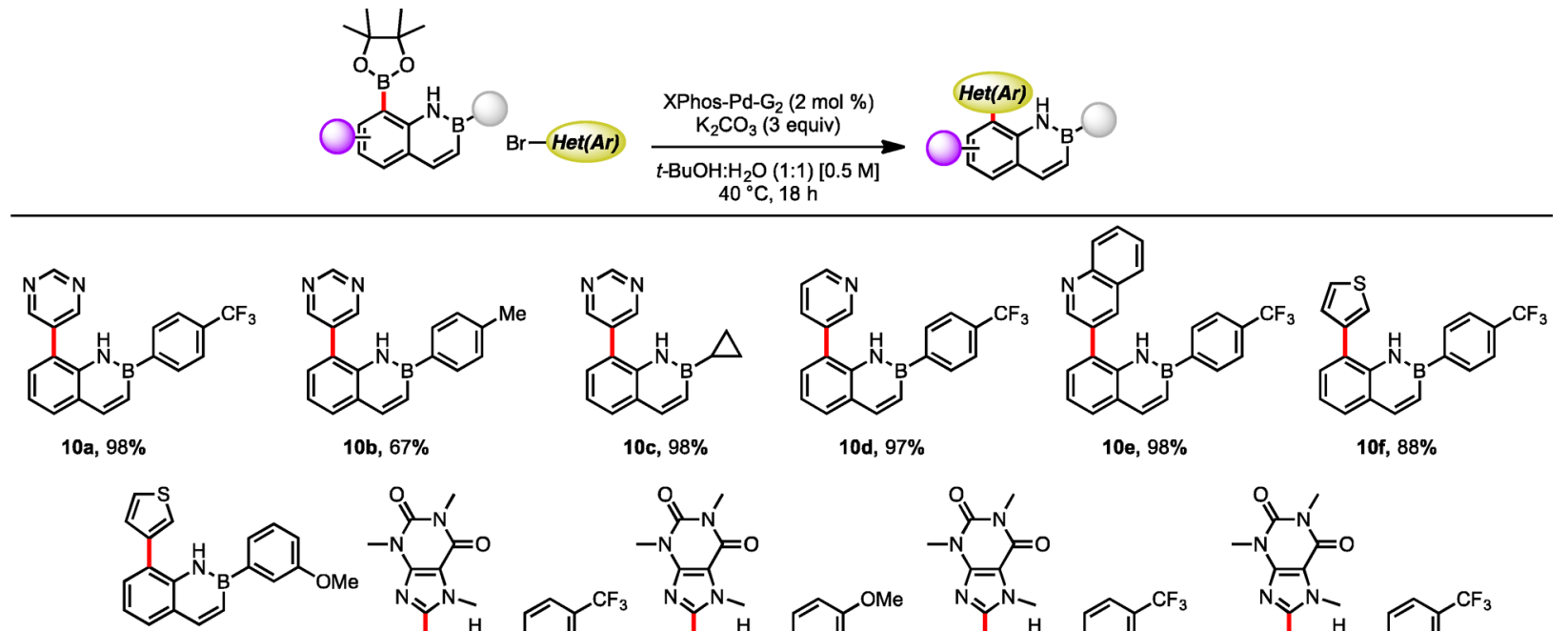

$10 \mathrm{~g}, 95 \%$

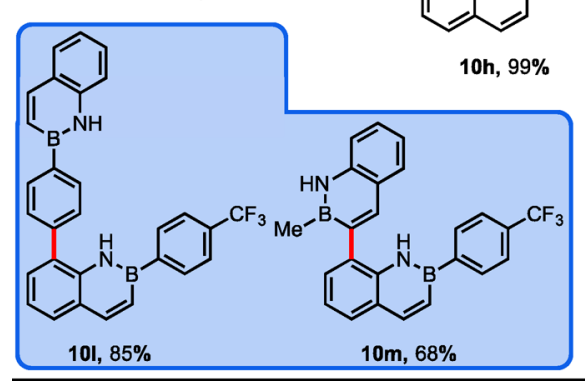<smiles>COc1ccc(B2C=Cc3cccc(-c4nc5c(c(=O)n(C)c(=O)n5C)n4C)c3N2)cc1</smiles><smiles></smiles>

$10 \mathrm{j}, 65 \%$<smiles>Cn1c(=O)c2c(nc(-c3cc(OC(F)(F)F)cc4c3NB(c3ccc(C(F)(F)F)cc3)C=C4)n2C)n(C)c1=O</smiles>

$10 k, 58 \%$

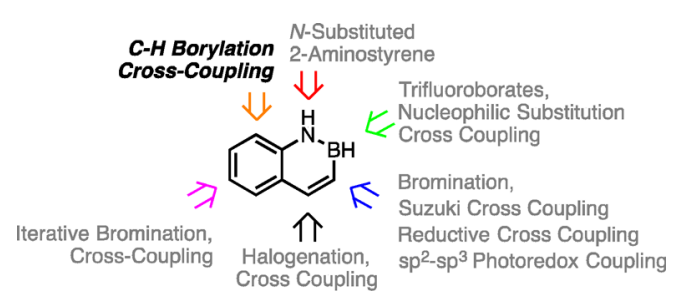

Figure 8. Methods of diversification for 2,1-borazaronaphthalene.

\section{EXPERIMENTAL SECTION}

General Considerations. All reactions were carried out under an inert atmosphere of nitrogen or argon in oven-dried glassware, unless otherwise noted. Toluene was dried using a J. C. Meyer solvent system. Methylcyclohexane was degassed under a stream of argon. All reagents were purchased commercially and used as received, unless otherwise noted. Column chromatography was performed by Combiflash using RediSep Rf Gold Normal-Phase Silica columns. Melting points $\left({ }^{\circ} \mathrm{C}\right)$ are uncorrected. Mass spectra (ESI- or EI-TOF) were recorded using $\mathrm{CH}_{2} \mathrm{Cl}_{2}$ or $\mathrm{MeCN}$ as the solvent. IR spectra were recorded using FTIR-ATR of the neat oil or solid products. NMR spectra $\left({ }^{1} \mathrm{H},{ }^{13} \mathrm{C}\left\{{ }^{1} \mathrm{H}\right\},{ }^{11} \mathrm{~B},{ }^{19} \mathrm{~F}\left\{{ }^{1} \mathrm{H}\right\}\right)$ were performed at $298 \mathrm{~K} .{ }^{1} \mathrm{H}$ $(500.4 \mathrm{MHz})$ and ${ }^{13} \mathrm{C}\left\{{ }^{1} \mathrm{H}\right\}(125.8 \mathrm{MHz})$ NMR chemical shifts are reported relative to internal TMS $(\delta=0.00 \mathrm{ppm})$ or to residual protiated solvent. Any observed splitting in the ${ }^{13} \mathrm{C}\left\{{ }^{1} \mathrm{H}\right\}$ NMR spectra is due to ${ }^{13} \mathrm{C}-{ }^{19} \mathrm{~F}$ coupling. ${ }^{11} \mathrm{~B}(128.4 \mathrm{MHz})$ and ${ }^{19} \mathrm{~F}\left\{{ }^{1} \mathrm{H}\right\} \mathrm{NMR}$ $(470.8 \mathrm{MHz})$ chemical shifts were referenced to external $\mathrm{BF}_{3} \cdot \mathrm{Et}_{2} \mathrm{O}$ $(0.0 \mathrm{ppm})$ and $\mathrm{CFCl}_{3}(0.0 \mathrm{ppm})$, respectively. Data are presented as follows: chemical shift (ppm), multiplicity $(\mathrm{s}=$ singlet, $\mathrm{d}=$ doublet, $\mathrm{t}=$ triplet, $\mathrm{q}=$ quartet, sept = septet, $\mathrm{m}=$ multiplet, $\mathrm{br}=$ broad $)$, coupling constant $J(\mathrm{~Hz})$, and integration.

Unreported 2,1-Borazaronaphthalene Scaffolds. 2,1-Borazaronaphthalene structures were synthesized by conditions as previously reported. ${ }^{3 a}$ In cases where the $o$-aminostyrene was not available, $o$ - bromoaniline derivatives (1.0 equiv) were cross-coupled with vinyltrifluoroborate (1.1 equiv) using $\mathrm{PdCl}_{2} \cdot \mathrm{dppf}(10 \mathrm{~mol} \%)$ and $\mathrm{Cs}_{2} \mathrm{CO}_{3}$ (2.5 equiv) in THF/ $\mathrm{H}_{2} \mathrm{O}(9: 1-0.1 \mathrm{M})$ mixture at $70{ }^{\circ} \mathrm{C}$ for $16 \mathrm{~h}$. After consumption of the bromoaniline, the reaction was quenched with $\mathrm{H}_{2} \mathrm{O}$, extracted with $\mathrm{Et}_{2} \mathrm{O}$, dried $\left(\mathrm{MgSO}_{4}\right)$, and concentrated in vacuo. The resulting solids were subject to a flash plug of silica, eluting with a 1:1 mixture of hexane/EtOAc, and then concentrated in vacuo. The resulting crude $o$-aminostyrenes were then subjected to the azaborine cyclization reaction with $p$ (trifluoromethyl)phenyl-trifluoroborate (1.05 equiv) using $\mathrm{SiCl}_{4}(1.0$ equiv) and $\mathrm{Et}_{3} \mathrm{~N}$ (1.5 equiv) in CPME/toluene $(1: 1-0.25 \mathrm{M})$ at $60^{\circ} \mathrm{C}$ for $16 \mathrm{~h}$. The reactions were then subjected to a silica gel plug and flushed with 9:1 mixture of hexane/EtOAc and further purified by automated column chromatography if required.

7-Fluoro-2-(4-(trifluoromethyl)phenyl)-2,1-borazaronaphthalene (Starting Material 4e). Obtained as a light brown solid $(235.3 \mathrm{mg}$, $32 \%, 2.5 \mathrm{mmol}$ scale); mp: $114-115{ }^{\circ} \mathrm{C}$; ${ }^{1} \mathrm{H}$ NMR ( $\mathrm{CDCl}_{3}, 500.4$ MHz): $\delta 8.15$ (d, $J=11.4 \mathrm{~Hz}, 2 \mathrm{H}), 7.99(\mathrm{~d}, J=7.7 \mathrm{~Hz}, 2 \mathrm{H}), 7.73(\mathrm{~d}, J$ $=7.7 \mathrm{~Hz}, 2 \mathrm{H}), 7.66(\mathrm{dd}, J=8.4,6.3 \mathrm{~Hz}, 1 \mathrm{H}), 7.21(\mathrm{~d}, J=11.5 \mathrm{~Hz}$, $1 \mathrm{H}), 7.08(\mathrm{dd}, J=9.9,2.6 \mathrm{~Hz}, 1 \mathrm{H}), 6.99(\mathrm{td}, J=8.5,2.3 \mathrm{~Hz}, 1 \mathrm{H}) \mathrm{ppm}$; ${ }^{13} \mathrm{C}\left\{{ }^{1} \mathrm{H}\right\}$ NMR $\left(\mathrm{CDCl}_{3}, 125.8 \mathrm{MHz}\right): \delta 162.7(\mathrm{~d}, J=247.8 \mathrm{~Hz})$, 145.6, $141.0(\mathrm{~d}, J=10.9 \mathrm{~Hz}), 132.8,131.5(\mathrm{q}, J=31.3 \mathrm{~Hz}), 131.2(\mathrm{~d}, J$ $=9.9 \mathrm{~Hz}), 124.8(\mathrm{q}, J=4.0 \mathrm{~Hz}), 124.2(\mathrm{q}, J=271.9 \mathrm{~Hz}), 122.5,110.0$ $(\mathrm{d}, J=23.1 \mathrm{~Hz}), 104.2(\mathrm{~d}, J=24.0 \mathrm{~Hz}) \mathrm{ppm} ;{ }^{19} \mathrm{~F}\left\{{ }^{1} \mathrm{H}\right\} \mathrm{NMR}\left(\mathrm{CDCl}_{3}\right.$, 470.8 MHz): $\delta-62.8,-111.3 \mathrm{ppm} ;{ }^{11} \mathrm{~B}$ NMR $\left(\mathrm{CDCl}_{3}, 128.4 \mathrm{MHz}\right)$ : $\delta 35.0$ ppm; IR: $\nu=3394,1569,1326,1215,1165,1119,1106,1067$, 829, $700 \mathrm{~cm}^{-1}$; HRMS (EI) $\mathrm{m} / z$ calcd for $\mathrm{C}_{15} \mathrm{H}_{10} \mathrm{BNF}_{4}[\mathrm{M}]^{+}$ 291.0842, found 291.0869 .

6-(Cyano)-2-(4-(trifluoromethyl)phenyl)-2,1-borazaronaphthalene (Starting Material 4f). Starting from pure 5-cyano-2-aminostyrene, the product was obtained as light yellow solid $(202 \mathrm{mg}, 85 \%$, $0.5 \mathrm{mmol}$ scale); mp: $163-165{ }^{\circ} \mathrm{C} ;{ }^{1} \mathrm{H}$ NMR $\left(\mathrm{CDCl}_{3}, 500.4 \mathrm{MHz}\right): \delta$ $8.35(\mathrm{~s}, 1 \mathrm{H}), 8.17(\mathrm{~d}, J=11.6 \mathrm{~Hz}, 1 \mathrm{H}), 8.06-8.00(\mathrm{~m}, 3 \mathrm{H}), 7.75(\mathrm{~d}, J$ $=7.8 \mathrm{~Hz}, 2 \mathrm{H}), 7.71(\mathrm{dd}, J=8.4,1.7 \mathrm{~Hz}, 1 \mathrm{H}), 7.47(\mathrm{~d}, J=8.4 \mathrm{~Hz}, 1 \mathrm{H})$, $7.40(\mathrm{dd}, J=11.6,1.7 \mathrm{~Hz}, 1 \mathrm{H}) \mathrm{ppm} ;{ }^{13} \mathrm{C}\left\{{ }^{1} \mathrm{H}\right\}$ NMR $\left(\mathrm{CDCl}_{3}, 125.8\right.$ $\mathrm{MHz}): \delta 145.1,142.4,134.5,133.0,131.9(\mathrm{q}, J=32.5 \mathrm{~Hz}), 130.9$, 
125.6, $124.9(\mathrm{q}, J=3.9 \mathrm{~Hz}), 124.1(\mathrm{q}, J=272.2 \mathrm{~Hz}), 119.3,119.0$, 104.9 ppm; ${ }^{19} \mathrm{~F}\left\{{ }^{1} \mathrm{H}\right\}$ NMR $\left(\mathrm{CDCl}_{3}, 470.8 \mathrm{MHz}\right): \delta-62.9 ;{ }^{11} \mathrm{~B}$ NMR $\left(\mathrm{CDCl}_{3}, 128.4 \mathrm{MHz}\right): \delta 34.6$; IR: $\nu=3307,2231,1614,1574,1324$, $1104,1066,834,800 \mathrm{~cm}^{-1}$; HRMS (EI) $\mathrm{m} / z$ calcd for $\mathrm{C}_{16} \mathrm{H}_{10} \mathrm{BF}_{3} \mathrm{~N}_{2}$ $[\mathrm{M}]^{+}$298.0889, found 298.0890 .

6-(Trifluoromethyl)-2-(4-(trifluoromethyl)phenyl)-2,1-borazaronaphthalene (Starting Material $\mathbf{4 g}$ ). Obtained as a light brown solid (471.6 mg, 55\%, $2.5 \mathrm{mmol} \mathrm{scale);} \mathrm{mp:} 109-110{ }^{\circ} \mathrm{C}$; ${ }^{1} \mathrm{H}$ NMR $\left(\mathrm{CDCl}_{3}, 500.4 \mathrm{MHz}\right): \delta 8.26(\mathrm{~s}, 1 \mathrm{H}), 8.19(\mathrm{~d}, J=11.5 \mathrm{~Hz}, 1 \mathrm{H}), 8.05-$ $7.94(\mathrm{~m}, 3 \mathrm{H}), 7.75(\mathrm{~d}, J=7.8 \mathrm{~Hz}, 2 \mathrm{H}), 7.70(\mathrm{dd}, J=8.5,1.9 \mathrm{~Hz}, 1 \mathrm{H})$, $7.45(\mathrm{~d}, J=8.5 \mathrm{~Hz}, 1 \mathrm{H}), 7.36(\mathrm{dd}, J=11.5,1.9 \mathrm{~Hz}, 1 \mathrm{H}) \mathrm{ppm} ;{ }^{13} \mathrm{C}$ $\left\{{ }^{1} \mathrm{H}\right\} \operatorname{NMR}\left(\mathrm{CDCl}_{3}, 125.8 \mathrm{MHz}\right): \delta 145.8,141.7,132.9,131.7(\mathrm{q}, J=$ $32.4 \mathrm{~Hz}), 127.0(\mathrm{q}, J=3.9 \mathrm{~Hz}), 125.0,124.9(\mathrm{q}, J=3.7 \mathrm{~Hz}), 124.8(\mathrm{q}$, $J=3.9 \mathrm{~Hz}), 124.3(\mathrm{q}, J=271.5 \mathrm{~Hz}), 124.2(\mathrm{q}, J=271.8 \mathrm{~Hz}), 123.7(\mathrm{q}$, $J=32.6 \mathrm{~Hz}), 118.8 \mathrm{ppm} ;{ }^{19} \mathrm{~F}\left\{{ }^{1} \mathrm{H}\right\} \operatorname{NMR}\left(\mathrm{CDCl}_{3}, 470.8 \mathrm{MHz}\right): \delta$ $-61.4,-62.8 \mathrm{ppm} ;{ }^{11} \mathrm{~B}$ NMR $\left(\mathrm{CDCl}_{3}, 128.4 \mathrm{MHz}\right): \delta 35.5 \mathrm{ppm}$; IR: $\nu$ $=3398,1627,1573,1318,1172,1119,1105,1065,806 \mathrm{~cm}^{-1}$; HRMS (EI) $m / z$ calcd for $\mathrm{C}_{16} \mathrm{H}_{10} \mathrm{BNF}_{6}[\mathrm{M}]^{+} 341.0810$, found 341.0829 .

6-Isopropyl-2-(4-(trifluoromethyl)phenyl)-2,1-borazaronaphthalene (Starting Material 4h). Starting from pure 5-isopropyl-2aminostyrene. Obtained as a white solid $(145.1 \mathrm{mg}, 79 \%, 0.5 \mathrm{mmol}$ scale); mp: $200-203{ }^{\circ} \mathrm{C} ;{ }^{1} \mathrm{H}$ NMR $\left(\mathrm{CDCl}_{3}, 500.4 \mathrm{MHz}\right): \delta 8.22-8.11$ $(\mathrm{m}, 2 \mathrm{H}), 8.00(\mathrm{~d}, J=7.7 \mathrm{~Hz}, 2 \mathrm{H}), 7.73(\mathrm{~d}, J=7.8 \mathrm{~Hz}, 2 \mathrm{H}), 7.54(\mathrm{~s}$, $1 \mathrm{H}), 7.40(\mathrm{dd}, J=8.6,1.6 \mathrm{~Hz}, 1 \mathrm{H}), 7.33(\mathrm{~d}, J=8.3 \mathrm{~Hz}, 1 \mathrm{H}), 7.25$ (dd, $J=11.6,1.6 \mathrm{~Hz}, 1 \mathrm{H}), 3.05(\mathrm{sept}, J=7.0 \mathrm{~Hz}, 1 \mathrm{H}), 1.35(\mathrm{~d}, J=6.9 \mathrm{~Hz}$, $6 \mathrm{H}) \mathrm{ppm} ;{ }^{13} \mathrm{C}\left\{{ }^{1} \mathrm{H}\right\}$ NMR $\left(\mathrm{CDCl}_{3}, 125.8 \mathrm{MHz}\right): \delta$ 146.1, 142.0, $138.1,132.7,131.1(\mathrm{q}, J=32.1 \mathrm{~Hz}), 127.5,126.5,125.7,124.7(\mathrm{q}, J=$ $3.4 \mathrm{~Hz}), 124.3(\mathrm{q}, J=272.0 \mathrm{~Hz}), 118.2,33.6,24.1 \mathrm{ppm} ;{ }^{19} \mathrm{~F}\left\{{ }^{1} \mathrm{H}\right\}$ NMR $\left(\mathrm{CDCl}_{3}, 470.8 \mathrm{MHz}\right): \delta-62.7 \mathrm{ppm} ;{ }^{11} \mathrm{~B} \mathrm{NMR}\left(\mathrm{CDCl}_{3}, 128.4\right.$ $\mathrm{MHz}): \delta 32.9$ ppm; IR: $\nu=3357,1571,1332,1162,1134,1120,1104$, 1066, $789 \mathrm{~cm}^{-1}$; HRMS (EI) $\mathrm{m} / z$ calcd for $\mathrm{C}_{18} \mathrm{H}_{17} \mathrm{BNF}_{3}[\mathrm{M}]^{+}$ 315.1406, found 315.1424 .

6-(Trifluoromethoxy)-2-(4-(trifluoromethyl)phenyl)-2,1-borazaronaphthalene (Starting Material 4i). Starting from pure 5trifluoromethoxy-2-aminostyrene. Obtained as a white solid (637 $\mathrm{mg}, 59 \%$, $3.0 \mathrm{mmol} \mathrm{scale})$; mp: $81-82{ }^{\circ} \mathrm{C}$; ${ }^{1} \mathrm{H}$ NMR $\left(\mathrm{CDCl}_{3}, 500.4\right.$ $\mathrm{MHz}): \delta 8.20(\mathrm{~s}, 1 \mathrm{H}), 8.14(\mathrm{~d}, J=11.5 \mathrm{~Hz}, 1 \mathrm{H}), 7.99(\mathrm{~d}, J=7.8 \mathrm{~Hz}$, $2 \mathrm{H}), 7.74(\mathrm{~d}, J=7.8 \mathrm{~Hz}, 2 \mathrm{H}), 7.56(\mathrm{~s}, 1 \mathrm{H}), 7.42-7.30(\mathrm{~m}, 3 \mathrm{H}) \mathrm{ppm}$; ${ }^{13} \mathrm{C}\left\{{ }^{1} \mathrm{H}\right\}$ NMR $\left(\mathrm{CDCl}_{3}, 125.8 \mathrm{MHz}\right): \delta 145.3,143.2,138.2,132.8$, $131.5(\mathrm{q}, J=32.2 \mathrm{~Hz}), 126.0,125.0(\mathrm{q}, J=3.8 \mathrm{~Hz}), 124.2(\mathrm{q}, J=272.1$ $\mathrm{Hz}), 122.0,121.1,120.6(\mathrm{q}, J=256.6 \mathrm{~Hz}), 119.4 \mathrm{ppm} ;{ }^{19} \mathrm{~F}\left\{{ }^{1} \mathrm{H}\right\} \mathrm{NMR}$ $\left(\mathrm{CDCl}_{3}, 470.8 \mathrm{MHz}\right): \delta-58.1,-62.8 \mathrm{ppm} ;{ }^{11} \mathrm{~B}$ NMR $\left(\mathrm{CDCl}_{3}, 128.4\right.$ $\mathrm{MHz}): \delta 33.7 \mathrm{ppm}$; IR: $\nu=3407,1565,1333,1260,1209,1153,1115$, $1102795,713 \mathrm{~cm}^{-1}$; HRMS (EI) $\mathrm{m} / z$ calcd for $\mathrm{C}_{16} \mathrm{H}_{10} \mathrm{BNOF}_{6}[\mathrm{M}]^{+}$ 357.0760, found 357.0778.

N-Methyl-2-(4-(trifluoromethyl)phenyl)-2,1-borazaronaphthalene (8). Starting from pure $\mathrm{N}$-methyl-2-aminostyrene. Obtained as a light brown solid (658 mg, 57\%, $4.0 \mathrm{mmol} \mathrm{scale})$; mp: $120-122{ }^{\circ} \mathrm{C}$; ${ }^{1} \mathrm{H}$ NMR $\left(\mathrm{CDCl}_{3}, 500.4 \mathrm{MHz}\right): \delta 8.12(\mathrm{~d}, J=11.3 \mathrm{~Hz}, 1 \mathrm{H}), 7.76(\mathrm{~d}, J$ $=7.9 \mathrm{~Hz}, 1 \mathrm{H}), 7.74(\mathrm{~d}, J=8.6 \mathrm{~Hz}, 2 \mathrm{H}), 7.72(\mathrm{~d}, J=8.2 \mathrm{~Hz}, 2 \mathrm{H})$, $7.69-7.60(\mathrm{~m}, 2 \mathrm{H}), 7.34(\mathrm{t}, J=7.2 \mathrm{~Hz}, 1 \mathrm{H}), 6.94(\mathrm{~d}, J=11.3 \mathrm{~Hz}, 1 \mathrm{H})$, $3.73(\mathrm{~s}, 3 \mathrm{H}) \mathrm{ppm} ;{ }^{13} \mathrm{C}\left\{{ }^{1} \mathrm{H}\right\}$ NMR $\left(\mathrm{CDCl}_{3}, 125.8 \mathrm{MHz}\right): \delta$ 145.2, $142.0,133.1,130.3,129.6(\mathrm{q}, J=32.1 \mathrm{~Hz}), 128.9,126.8,124.4(\mathrm{q}, J=$ $272.0 \mathrm{~Hz}), 124.2(\mathrm{q}, J=3.7 \mathrm{~Hz}), 121.2,115.1,36.7 \mathrm{ppm} ;{ }^{19} \mathrm{~F}\left\{{ }^{1} \mathrm{H}\right\}$ $\operatorname{NMR}\left(\mathrm{CDCl}_{3}, 470.8 \mathrm{MHz}\right): \delta-62.6 \mathrm{ppm} ;{ }^{11} \mathrm{~B} \mathrm{NMR}\left(\mathrm{CDCl}_{3}, 128.4\right.$ $\mathrm{MHz}): \delta 35.8$ ppm; IR: $\nu=3020,1589,1552,1325,1159,1112,1101$, $1067,805,762 \mathrm{~cm}^{-1}$; HRMS (EI) $\mathrm{m} / z$ calcd for $\mathrm{C}_{16} \mathrm{H}_{13} \mathrm{BNF}_{3}[\mathrm{M}]^{+}$ 287.1093, found 287.1083.

Deuteration of 2,1-borazaronaphthalene N-Deuterio-2-(4(trifluoromethyl)phenyl)-2,1-borazaronaphthalene (6). To a microwave vial with a stir bar, 2-(4-(trifluoromethyl)phenyl)-2,1-borazaronaphthalene (273 mg, $1 \mathrm{mmol}$, 1 equiv) was added. The vial was capped and purged with argon. $\mathrm{Et}_{2} \mathrm{O}(3 \mathrm{~mL})$ was added followed by KHMDS ( $2 \mathrm{~mL}$ of a $1 \mathrm{M}$ solution in THF, 2 equiv). The reaction was stirred for $20 \mathrm{~min}$ at $\mathrm{rt}$, at which time $\mathrm{D}_{2} \mathrm{O}(3 \mathrm{~mL})$ was added dropwise and stirred for an additional $30 \mathrm{~min}$. The reaction mixture was then extracted with EtOAc $(5 \mathrm{~mL})$ and dried $\left(\mathrm{MgSO}_{4}\right)$. Removal of the solvent afforded $218 \mathrm{mg}$ (80\%) of a yellow solid; mp: 149-150 ${ }^{\circ} \mathrm{C}$; ${ }^{1} \mathrm{H}$ NMR $\left(\mathrm{CDCl}_{3}, 500.4 \mathrm{MHz}\right): \delta 8.19(\mathrm{~d}, J=11.5 \mathrm{~Hz}, 1 \mathrm{H}), 8.00$ $(\mathrm{d}, J=7.7 \mathrm{~Hz}, 2 \mathrm{H}), 7.71(\mathrm{~d}, J=7.8 \mathrm{~Hz}, 2 \mathrm{H}), 7.69(\mathrm{~d}, J=7.9 \mathrm{~Hz}, 1 \mathrm{H})$, $7.48(\mathrm{t}, J=7.2 \mathrm{~Hz}, 1 \mathrm{H}), 7.37(\mathrm{~d}, J=8.1 \mathrm{~Hz}, 1 \mathrm{H}), 7.30-7.19(\mathrm{~m}, 2 \mathrm{H})$ ppm; ${ }^{13} \mathrm{C}\left\{{ }^{1} \mathrm{H}\right\}$ NMR $\left(\mathrm{CDCl}_{3}, 125.8 \mathrm{MHz}\right): \delta$ 146.1, 139.7, 132.8, $131.2(\mathrm{q}, J=32.1 \mathrm{~Hz}), 129.5,128.6,125.7,124.7(\mathrm{q}, J=3.7 \mathrm{~Hz}), 124.3$ $(\mathrm{q}, J=272.0 \mathrm{~Hz}), 121.4,118.2 \mathrm{ppm} ;{ }^{19} \mathrm{~F}\left\{{ }^{1} \mathrm{H}\right\}$ NMR $\left(\mathrm{CDCl}_{3}, 470.8\right.$ $\mathrm{MHz}): \delta-62.7 \mathrm{ppm} ;{ }^{11} \mathrm{~B}$ NMR $\left(\mathrm{CDCl}_{3}, 128.4 \mathrm{MHz}\right): \delta 33.0 \mathrm{ppm}$; IR: $\nu=2923,2502,1612,1325,1118,1108,1071,812,762,643 \mathrm{~cm}^{-1}$; HRMS (EI) $m / z$ calcd for $\mathrm{C}_{15} \mathrm{H}_{10} \mathrm{DBNF}_{3}[\mathrm{M}]^{+}$274.0999, found 274.1018.

Experimental Procedure for Iridium-Catalyzed $\mathrm{C}-\mathrm{H}$ Borylation of 2,1-Borazaronaphthalenes. In a microwave vial with stir bar, 2,1-borazaronaphthalene ( $0.5 \mathrm{mmol}, 1.0$ equiv) and $\mathrm{B}_{2} \mathrm{Pin}_{2}(133.3$ $\mathrm{mg}, 0.53 \mathrm{mmol}, 1.05$ equiv) were added. The reaction vessel was capped and purged with argon followed by the addition of $1 \mathrm{~mL}$ of degassed methylcyclohexane. In a separate vial, $[\operatorname{Ir}(\mu \text {-OMe })(\mathrm{COD})]_{2}$ $(6.6 \mathrm{mg}, 0.01 \mathrm{mmol}, 2 \mathrm{~mol} \%)$ and di-t-Bu-bipyridine $(6.7 \mathrm{mg}, 0.025$ $\mathrm{mmol}, 5 \mathrm{~mol} \%)$ were precomplexed under an inert atmosphere in 1 $\mathrm{mL}$ degassed methylcyclohexane, stirring $30 \mathrm{~min}$ at $\mathrm{rt}$ as the mixture turned magenta. The catalyst mixture was then added to the reaction mixture via syringe. The reaction was then heated and run for $16 \mathrm{~h}$ while being monitored by HPLC for completion. Upon cooling, the reaction mixture was condensed and adhered to silica, which was directly subjected to purification by automated silica gel column chromatography with a gradient solution of hexane/EtOAc as the mobile phase.

8-(4,4,5,5-Tetramethyl-1,3,2-dioxaboryl)-2-(4-(trifluoromethyl)phenyl)-2,1-borazaronaphthalene (3a). Reaction was run at $40{ }^{\circ} \mathrm{C}$, and the product was obtained as a white solid $(166 \mathrm{mg}, 83 \%, 0.5 \mathrm{mmol}$ scale); mp: $135-137{ }^{\circ} \mathrm{C} ;{ }^{1} \mathrm{H}$ NMR $\left(\mathrm{CDCl}_{3}, 500.4 \mathrm{MHz}\right): \delta 10.61(\mathrm{~s}$, $1 \mathrm{H}), 8.21(\mathrm{~d}, J=11.4 \mathrm{~Hz}, 1 \mathrm{H}), 8.12(\mathrm{~d}, J=7.7 \mathrm{~Hz}, 2 \mathrm{H}), 8.05(\mathrm{~d}, J=$ $7.2 \mathrm{~Hz}, 1 \mathrm{H}), 7.82(\mathrm{~d}, J=7.8 \mathrm{~Hz}, 1 \mathrm{H}), 7.78(\mathrm{~d}, J=7.6 \mathrm{~Hz}, 2 \mathrm{H}), 7.32$ $(\mathrm{d}, J=11.5 \mathrm{~Hz}, 1 \mathrm{H}), 7.29-7.23(\mathrm{~m}, 1 \mathrm{H}), 1.51(\mathrm{~s}, 12 \mathrm{H}) \mathrm{ppm} ;{ }^{13} \mathrm{C}$ $\left\{{ }^{1} \mathrm{H}\right\}$ NMR $\left(\mathrm{CDCl}_{3}, 125.8 \mathrm{MHz}\right): \delta 146.3,145.6,137.0,133.4,133.0$, $131.1(\mathrm{q}, J=32.0 \mathrm{~Hz}), 125.3,124.7(\mathrm{q}, J=4.0 \mathrm{~Hz}), 124.4(\mathrm{q}, J=272.1$ $\mathrm{Hz}), 120.6,84.3,25.0 \mathrm{ppm} ;{ }^{19} \mathrm{~F}\left\{{ }^{1} \mathrm{H}\right\} \operatorname{NMR}\left(\mathrm{CDCl}_{3}, 470.8 \mathrm{MHz}\right): \delta$ -62.7 ppm; ${ }^{11} \mathrm{~B}$ NMR $\left(\mathrm{CDCl}_{3}, 128.4 \mathrm{MHz}\right): \delta 30.8$ (br, 2B) ppm; IR: $\nu=3355,2984,1600,1321,1307,1127,1104,786 \mathrm{~cm}^{-1}$; HRMS (EI) $m / z$ calcd for $\mathrm{C}_{21} \mathrm{H}_{22} \mathrm{~B}_{2} \mathrm{NO}_{2} \mathrm{~F}_{3}[\mathrm{M}]^{+} 399.1789$, found 399.1798.

2-(p-Tolyl)-8-(4,4,5,5-tetramethyl-1,3,2-dioxaboryl)-2,1-borazaronaphthalene (3b). Reaction was run at $80{ }^{\circ} \mathrm{C}$ for $24 \mathrm{~h}$, and the product was obtained as a white solid $(552 \mathrm{mg}, 80 \%, 2.0 \mathrm{mmol} \mathrm{scale})$; mp: $105-108{ }^{\circ} \mathrm{C} ;{ }^{1} \mathrm{H}$ NMR $\left(\mathrm{CDCl}_{3}, 500.4 \mathrm{MHz}\right): \delta 10.46(\mathrm{~s}, 1 \mathrm{H})$, $8.12(\mathrm{~d}, J=11.5 \mathrm{~Hz}, 1 \mathrm{H}), 7.97(\mathrm{dd}, J=7.2,1.4 \mathrm{~Hz}, 1 \mathrm{H}), 7.92(\mathrm{~d}, J=$ $7.8 \mathrm{~Hz}, 2 \mathrm{H}), 7.76(\mathrm{~d}, J=7.6 \mathrm{~Hz}, 1 \mathrm{H}), 7.38-7.30(\mathrm{~m}, 3 \mathrm{H}), 7.18(\mathrm{t}, J=$ $7.4 \mathrm{~Hz}, 1 \mathrm{H}), 2.44(\mathrm{~s}, 3 \mathrm{H}), 1.47(\mathrm{~s}, 12 \mathrm{H}) \mathrm{ppm} ;{ }^{13} \mathrm{C}\left\{{ }^{1} \mathrm{H}\right\}$ NMR $\left(\mathrm{CDCl}_{3}, 125.8 \mathrm{MHz}\right): \delta 145.9,145.5,139.4,136.6,133.3,132.9,132.6$, 128.9, 119.9, 84.1, 25.0, 21.5 ppm; ${ }^{11} \mathrm{~B}$ NMR $\left(\mathrm{CDCl}_{3}, 128.4 \mathrm{MHz}\right): \delta$ $33.2,30.5$ ppm; IR: $\nu=3351,2973,1599,1565,1355,1307,1138,775$ $\mathrm{cm}^{-1}$; HRMS (EI) $m / z$ calcd for $\mathrm{C}_{21} \mathrm{H}_{25} \mathrm{~B}_{2} \mathrm{NO}_{2}[\mathrm{M}]^{+}$345.2071, found 345.2077.

2-Phenyl-8-(4,4,5,5-tetramethyl-1,3,2-dioxaboryl)-2,1-borazaronaphthalene (3c). Reaction was run at $40{ }^{\circ} \mathrm{C}$, and the product was obtained as a white solid (104 mg, 63\%, $0.5 \mathrm{mmol} \mathrm{scale);} \mathrm{mp:} \mathrm{131-}$ $133{ }^{\circ} \mathrm{C} ;{ }^{1} \mathrm{H}$ NMR $\left(\mathrm{CDCl}_{3}, 500.4 \mathrm{MHz}\right): \delta 10.58(\mathrm{~s}, 1 \mathrm{H}), 8.20(\mathrm{~d}, J=$ $11.5 \mathrm{~Hz}, 1 \mathrm{H}), 8.09$ (d, $J=7.4 \mathrm{~Hz}, 2 \mathrm{H}), 8.06(\mathrm{~d}, J=7.2 \mathrm{~Hz}, 1 \mathrm{H}), 7.83$ $(\mathrm{d}, J=7.8 \mathrm{~Hz}, 1 \mathrm{H}), 7.62-7.51(\mathrm{~m}, 3 \mathrm{H}), 7.38(\mathrm{dd}, J=11.5,1.9 \mathrm{~Hz}$, $1 \mathrm{H}), 7.26(\mathrm{t}, J=7.4 \mathrm{~Hz}, 1 \mathrm{H}), 1.53(\mathrm{~s}, 12 \mathrm{H}) \mathrm{ppm} ;{ }^{13} \mathrm{C}\left\{{ }^{1} \mathrm{H}\right\} \mathrm{NMR}$ $\left(\mathrm{CDCl}_{3}, 125.8 \mathrm{MHz}\right): \delta 145.9,145.7,136.8,133.4,132.9,129.5,128.2$, 125.3, 120.2, 84.2, $25.0 \mathrm{ppm}$; ${ }^{11} \mathrm{~B}$ NMR $\left(\mathrm{CDCl}_{3}, 128.4 \mathrm{MHz}\right): \delta 30.8$ (br, 2B) ppm; IR: $\nu=3348,2981,1599,1354,1305,1138,1125$, 793, $746 \mathrm{~cm}^{-1}$; HRMS (EI) $\mathrm{m} / z$ calcd for $\mathrm{C}_{20} \mathrm{H}_{23} \mathrm{~B}_{2} \mathrm{NO}_{2}[\mathrm{M}]^{+} 331.1915$, found 331.1921.

2-(4-Methoxyphenyl)-8-(4,4,5,5-tetramethyl-1,3,2-dioxaboryl)2,1-borazaronaphthalene ( $3 d$ ). Reaction was run at $40{ }^{\circ} \mathrm{C}$, and the product was obtained as an off-white solid $(144 \mathrm{mg}, 80 \%, 0.5 \mathrm{mmol}$ scale); mp: $95-98{ }^{\circ} \mathrm{C} ;{ }^{1} \mathrm{H}$ NMR $\left(\mathrm{CDCl}_{3}, 500.4 \mathrm{MHz}\right): \delta 10.40(\mathrm{~s}$, $1 \mathrm{H}), 8.11(\mathrm{~d}, J=11.5 \mathrm{~Hz}, 1 \mathrm{H}), 8.01-7.95(\mathrm{~m}, 3 \mathrm{H}), 7.76(\mathrm{dd}, J=7.9$, $1.6 \mathrm{~Hz}, 1 \mathrm{H}), 7.27(\mathrm{~s}, 1 \mathrm{H}), 7.18(\mathrm{t}, J=7.4 \mathrm{~Hz}, 1 \mathrm{H}), 7.06(\mathrm{~d}, J=8.3 \mathrm{~Hz}$, $2 \mathrm{H}), 3.91(\mathrm{~s}, 3 \mathrm{H}), 1.49(\mathrm{~s}, 12 \mathrm{H}) \mathrm{ppm} ;{ }^{13} \mathrm{C}\left\{{ }^{1} \mathrm{H}\right\} \mathrm{NMR}\left(\mathrm{CDCl}_{3}, 125.8\right.$ $\mathrm{MHz}): \delta 160.9,146.0,145.3,136.6,134.4,133.3,125.1,119.9,113.7$, 84.1, 55.1, 25.0 ppm; ${ }^{11} \mathrm{~B}$ NMR $\left(\mathrm{CDCl}_{3}, 128.4 \mathrm{MHz}\right): \delta 31.4$ (br, 2B) 
ppm; IR: $\nu=3354,2975,1597,1355,1308,1252,1178,1138,1125$, $800,788 \mathrm{~cm}^{-1}$; HRMS (ESI) $\mathrm{m} / z$ calcd for $\mathrm{C}_{21} \mathrm{H}_{26} \mathrm{~B}_{2} \mathrm{NO}_{3}[\mathrm{M}+\mathrm{H}]^{+}$ 362.2099 , found 362.2109 .

2-(3-Methoxyphenyl)-8-(4,4,5,5-tetramethyl-1,3,2-dioxaboryl)2,1-borazaronaphthalene (3e). Reaction was run at $80^{\circ} \mathrm{C}$, and the product was obtained as a white solid ( $91 \mathrm{mg}, 61 \%, 0.41 \mathrm{mmol} \mathrm{scale})$; mp: 96-98 ${ }^{\circ} \mathrm{C} ;{ }^{1} \mathrm{H}$ NMR $\left(\mathrm{CDCl}_{3}, 500.4 \mathrm{MHz}\right): \delta 10.48(\mathrm{~s}, 1 \mathrm{H}), 8.17$ (dd, $J=11.6,9.1 \mathrm{~Hz}, 1 \mathrm{H}), 8.02(\mathrm{t}, J=7.1 \mathrm{~Hz}, 1 \mathrm{H}), 7.84-7.75(\mathrm{~m}$, $1 \mathrm{H}), 7.63(\mathrm{~d}, J=7.4 \mathrm{~Hz}, 1 \mathrm{H}), 7.57(\mathrm{t}, J=4.4 \mathrm{~Hz}, 1 \mathrm{H}), 7.46(\mathrm{t}, J=7.8$ $\mathrm{Hz}, 1 \mathrm{H}), 7.35-7.28(\mathrm{~m}, 1 \mathrm{H}), 7.22(\mathrm{dd}, J=14.8,7.4 \mathrm{~Hz}, 1 \mathrm{H}), 7.05$ (dd, $J=8.3,2.8 \mathrm{~Hz}, 1 \mathrm{H}), 3.95$ (s, $3 \mathrm{H}), 1.49(\mathrm{~s}, 12 \mathrm{H}) \mathrm{ppm} ;{ }^{13} \mathrm{C}\left\{{ }^{1} \mathrm{H}\right\}$ NMR $\left(\mathrm{CDCl}_{3}, 125.8 \mathrm{MHz}\right): \delta 159.5,145.8,145.7,136.8,133.4,129.2$, 125.3 ( $2 \times \mathrm{C}), 120.2,117.9,115.2,84.1,55.1,25.0 \mathrm{ppm} ;{ }^{11} \mathrm{~B}$ NMR $\left(\mathrm{CDCl}_{3}, 128.4 \mathrm{MHz}\right): \delta 31.0$ (br, 2B) ppm; IR: $\nu=3355,2965,1603$, 1462, 1358, 1303, 1265, 1142, 1126, $774 \mathrm{~cm}^{-1}$; HRMS (ESI) $\mathrm{m} / z$ calcd for $\mathrm{C}_{21} \mathrm{H}_{26} \mathrm{~B}_{2} \mathrm{NO}_{3}[\mathrm{M}+\mathrm{H}]^{+} 362.2099$, found 362.2092.

2-(2,3-Dihydrobenzo[1,4]dioxine)-8-(4,4,5,5-tetramethyl-1,3,2-dioxaboryl)-2, 1-borazaronaphthalene (3f). Reaction was run at $40{ }^{\circ} \mathrm{C}$, and the product was obtained as a yellow oil $(162 \mathrm{mg}, 83 \%, 0.5 \mathrm{mmol}$ scale); ${ }^{1} \mathrm{H}$ NMR $\left(\mathrm{CDCl}_{3}, 500.4 \mathrm{MHz}\right): \delta 10.46(\mathrm{~s}, 1 \mathrm{H}), 8.14(\mathrm{~d}, J=$ $11.6 \mathrm{~Hz}, 1 \mathrm{H}), 8.01(\mathrm{dd}, J=7.2,1.6 \mathrm{~Hz}, 1 \mathrm{H}), 7.79(\mathrm{dd}, J=7.7,1.6 \mathrm{~Hz}$, $1 \mathrm{H}), 7.58(\mathrm{~d}, J=1.6 \mathrm{~Hz}, 1 \mathrm{H}), 7.56(\mathrm{dd}, J=7.9,1.6 \mathrm{~Hz}, 1 \mathrm{H}), 7.29(\mathrm{dd}$, $J=11.7,1.9 \mathrm{~Hz}, 1 \mathrm{H}), 7.21(\mathrm{t}, J=7.4 \mathrm{~Hz}, 1 \mathrm{H}), 7.05(\mathrm{~d}, J=7.9 \mathrm{~Hz}$, $1 \mathrm{H}), 4.35(\mathrm{~s}, 4 \mathrm{H}), 1.52(\mathrm{~s}, 12 \mathrm{H}) \mathrm{ppm} ;{ }^{13} \mathrm{C}\left\{{ }^{1} \mathrm{H}\right\} \mathrm{NMR}\left(\mathrm{CDCl}_{3}, 125.8\right.$ $\mathrm{MHz}): \delta 146.0,145.4,145.1,143.6,136.6,133.3,126.2,125.1,121.7$, 112.0, 117.2, 84.2, 64.6, 64.3, 25.0 ppm; ${ }^{11} \mathrm{~B}$ NMR $\left(\mathrm{CDCl}_{3}, 128.4\right.$ $\mathrm{MHz}$ ): $\delta 30.5$ (br, 2B) ppm; IR: $\nu=3347,2981,1598,1309,1281$, $1126,1066,801,795 \mathrm{~cm}^{-1}$; HRMS (ESI) $m / z$ calcd for $\mathrm{C}_{22} \mathrm{H}_{25} \mathrm{~B}_{2} \mathrm{NO}_{4}$ $[\mathrm{M}]^{+}$389.1970, found 389.1987 .

2-(4-lodophenyl)-8-(4,4,5,5-tetramethyl-1,3,2-dioxaboryl)-2,1borazaronaphthalene $(3 \mathrm{~g})$. Reaction was run at $40{ }^{\circ} \mathrm{C}$, and the product was obtained as an off-while solid $(139 \mathrm{mg}, 61 \%, 0.5 \mathrm{mmol}$ scale); mp: $114-115{ }^{\circ} \mathrm{C} ;{ }^{1} \mathrm{H}$ NMR $\left(\mathrm{CDCl}_{3}, 500.4 \mathrm{MHz}\right): \delta 10.48$ (s, $1 \mathrm{H}), 8.15(\mathrm{~d}, J=11.5 \mathrm{~Hz}, 1 \mathrm{H}), 7.99(\mathrm{dd}, J=7.1,1.6 \mathrm{~Hz}, 1 \mathrm{H}), 7.85(\mathrm{~d}$, $J=8.0 \mathrm{~Hz}, 2 \mathrm{H}), 7.78(\mathrm{dd}, J=7.7,1.5 \mathrm{~Hz}, 1 \mathrm{H}), 7.72(\mathrm{~d}, J=8.0 \mathrm{~Hz}$, $2 \mathrm{H}), 7.25(\mathrm{dd}, J=11.5,1.9 \mathrm{~Hz}, 1 \mathrm{H}), 7.21(\mathrm{t}, J=7.4 \mathrm{~Hz}, 1 \mathrm{H}), 1.47(\mathrm{~s}$, $12 \mathrm{H}) \mathrm{ppm} ;{ }^{13} \mathrm{C}\left\{{ }^{1} \mathrm{H}\right\}$ NMR $\left(\mathrm{CDCl}_{3}, 125.8 \mathrm{MHz}\right): \delta 146.0,145.7$, 137.2, 136.9, 134.5, 133.4, 125.2, 120.3, 96.4, 84.2, $25.0 \mathrm{ppm} ;{ }^{11} \mathrm{~B}$ NMR $\left(\mathrm{CDCl}_{3}, 128.4 \mathrm{MHz}\right): \delta 31.3(\mathrm{br}, 2 \mathrm{~B})$ ppm; IR: $\nu=3347,2984$, $1600,1352,1306,1124,792,775 \mathrm{~cm}^{-1}$; HRMS (EI) $\mathrm{m} / z$ calcd for $\mathrm{C}_{20} \mathrm{H}_{22} \mathrm{~B}_{2} \mathrm{NO}_{2} \mathrm{I}[\mathrm{M}+\mathrm{Na}]^{+} 457.0881$, found 457.0906 .

2-(4-Bromophenyl)-8-(4,4,5,5-tetramethyl-1,3,2-dioxaboryl)-2,1borazaronaphthalene (3h). Reaction was run at $40{ }^{\circ} \mathrm{C}$, and the product was obtained as a white solid $(164 \mathrm{mg}, 80 \%, 0.5 \mathrm{mmol} \mathrm{scale})$; mp: $132-135{ }^{\circ} \mathrm{C} ;{ }^{1} \mathrm{H}$ NMR $\left(\mathrm{CDCl}_{3}, 500.4 \mathrm{MHz}\right): \delta 10.53(\mathrm{~s}, 1 \mathrm{H})$, $8.18(\mathrm{~d}, J=11.5 \mathrm{~Hz}, 1 \mathrm{H}), 8.04(\mathrm{dd}, J=7.2,1.6 \mathrm{~Hz}, 1 \mathrm{H}), 7.89(\mathrm{~d}, J=$ $8.0 \mathrm{~Hz}, 2 \mathrm{H}), 7.81(\mathrm{dd}, J=7.8,1.5 \mathrm{~Hz}, 1 \mathrm{H}), 7.68(\mathrm{~d}, J=8.0 \mathrm{~Hz}, 2 \mathrm{H})$, 7.33-7.21 (m, 2H), $1.51(\mathrm{~s}, 12 \mathrm{H}) \mathrm{ppm} ;{ }^{13} \mathrm{C}\left\{{ }^{1} \mathrm{H}\right\}$ NMR $\left(\mathrm{CDCl}_{3}\right.$, $125.8 \mathrm{MHz}): \delta 146.0,145.7,136.9,134.4,133.4,131.3,125.3,124.2$, 120.4, 84.2, 25.0 ppm; ${ }^{11} \mathrm{~B}$ NMR $\left(\mathrm{CDCl}_{3}, 128.4 \mathrm{MHz}\right): \delta 30.4$ (br, 2B) ppm; IR: $\nu=3340,2976,1601,1353,1307,1138,1125,794,777$ $\mathrm{cm}^{-1}$; HRMS (ESI) $\mathrm{m} / z$ calcd for $\mathrm{C}_{20} \mathrm{H}_{23} \mathrm{~B}_{2} \mathrm{NO}_{2} \mathrm{Br}[\mathrm{M}+\mathrm{H}]^{+}$ 410.1098 , found 410.1092 .

2-(3-Bromophenyl)-8-(4,4,5,5-tetramethyl-1,3,2-dioxaboryl)-2,1borazaronaphthalene (3i). Reaction was run at $40{ }^{\circ} \mathrm{C}$, and the product was obtained as a white solid $(102 \mathrm{mg}, 50 \%, 0.5 \mathrm{mmol} \mathrm{scale})$; mp: $211-213{ }^{\circ} \mathrm{C} ;{ }^{1} \mathrm{H}$ NMR $\left(\mathrm{CDCl}_{3}, 500.4 \mathrm{MHz}\right): \delta 10.62(\mathrm{~s}, 1 \mathrm{H})$, 8.24-8.15 (m, 2H), $8.03(\mathrm{~d}, J=6.5 \mathrm{~Hz}, 1 \mathrm{H}), 7.96(\mathrm{~d}, J=7.0 \mathrm{~Hz}, 1 \mathrm{H})$, $7.81(\mathrm{~d}, J=7.7 \mathrm{~Hz}, 1 \mathrm{H}), 7.61(\mathrm{~d}, J=8.2 \mathrm{~Hz}, 1 \mathrm{H}), 7.39(\mathrm{t}, J=7.5 \mathrm{~Hz}$, 1H), 7.33-7.20 (m, 2H), $1.52(\mathrm{~s}, 12 \mathrm{H}) \mathrm{ppm} ;{ }^{13} \mathrm{C}\left\{{ }^{1} \mathrm{H}\right\} \mathrm{NMR}\left(\mathrm{CDCl}_{3}\right.$, $125.8 \mathrm{MHz}): \delta 146.1,145.7,136.9,135.4,133.4,132.2,131.6,129.9$, 125.3, 123.1, 120.4, 84.3, 25.0 ppm; ${ }^{11} \mathrm{~B}$ NMR $\left(\mathrm{CDCl}_{3}, 128.4 \mathrm{MHz}\right): \delta$ 31.3 (br, 2B) ppm; IR: $\nu=3354,2964,1602,1358,1307,1125,776$ $\mathrm{cm}^{-1}$; HRMS (ESI) $\mathrm{m} / z$ calcd for $\mathrm{C}_{20} \mathrm{H}_{23} \mathrm{~B}_{2} \mathrm{NO}_{2} \mathrm{Br}[\mathrm{M}+\mathrm{H}]^{+}$ 410.1098, found 410.1086 .

2-(2-Bromophenyl)-8-(4,4,5,5-tetramethyl-1,3,2-dioxaboryl)-2,1borazaronaphthalene (3j). Reaction was run at $40{ }^{\circ} \mathrm{C}$, and the product was obtained as a yellow solid (62 $\mathrm{mg}, 30 \%, 0.5 \mathrm{mmol} \mathrm{scale})$; mp: 89-90 ${ }^{\circ} \mathrm{C} ;{ }^{1} \mathrm{H}$ NMR $\left(\mathrm{CDCl}_{3}, 500.4 \mathrm{MHz}\right): \delta 10.42(\mathrm{~s}, 1 \mathrm{H}), 8.16$ $(\mathrm{d}, J=11.5 \mathrm{~Hz}, 1 \mathrm{H}), 8.02(\mathrm{~d}, J=6.5 \mathrm{~Hz}, 1 \mathrm{H}), 7.81(\mathrm{~d}, J=7.7 \mathrm{~Hz}$,
$1 \mathrm{H}), 7.67(\mathrm{dd}, J=11.5,7.3 \mathrm{~Hz}, 2 \mathrm{H}), 7.40(\mathrm{t}, J=7.6 \mathrm{~Hz}, 1 \mathrm{H}), 7.29-$ $7.21(\mathrm{~m}, 3 \mathrm{H}), 1.42(\mathrm{~s}, 12 \mathrm{H}) \mathrm{ppm} ;{ }^{13} \mathrm{C}\left\{{ }^{1} \mathrm{H}\right\}$ NMR $\left(\mathrm{CDCl}_{3}, 125.8\right.$ $\mathrm{MHz}): \delta 145.3,145.1,136.9,135.9,133.4,132.6,129.9,127.5,126.6$, 125.0, 120.5, 84.2, 24.9 ppm; ${ }^{11} \mathrm{~B}$ NMR $\left(\mathrm{CDCl}_{3}, 128.4 \mathrm{MHz}\right): \delta 31.8$ (br, 2B) ppm; IR: $\nu=3338,2991,1597,1354,1141,1126,819,757$ $\mathrm{cm}^{-1}$; HRMS (ESI) $\mathrm{m} / z$ calcd for $\mathrm{C}_{20} \mathrm{H}_{23} \mathrm{~B}_{2} \mathrm{NO}_{2} \mathrm{Br}[\mathrm{M}+\mathrm{H}]^{+}$ 410.1098 , found 410.1085 .

2-(3-Chlorophenyl)-8-(4,4,5,5-tetramethyl-1,3,2-dioxaboryl)-2,1borazaronaphthalene $(3 \mathrm{k})$. Reaction was run at $80{ }^{\circ} \mathrm{C}$, and the product was obtained as a white solid ( $56 \mathrm{mg}, 30 \%, 0.33 \mathrm{mmol} \mathrm{scale})$; mp: $118-120{ }^{\circ} \mathrm{C} ;{ }^{1} \mathrm{H}$ NMR $\left(\mathrm{CDCl}_{3}, 500.4 \mathrm{MHz}\right): \delta 10.60(\mathrm{~s}, 1 \mathrm{H})$, $8.18(\mathrm{~d}, J=11.6 \mathrm{~Hz}, 1 \mathrm{H}), 8.05-7.95(\mathrm{~m}, 2 \mathrm{H}), 7.92-7.86(\mathrm{~m}, 1 \mathrm{H})$, $7.80(\mathrm{~d}, J=7.8 \mathrm{~Hz}, 1 \mathrm{H}), 7.47-7.41(\mathrm{~m}, 2 \mathrm{H}), 7.30-7.27(\mathrm{~m}, 1 \mathrm{H}), 7.23$ $(\mathrm{t}, J=7.5 \mathrm{~Hz}, 1 \mathrm{H}), 1.50(\mathrm{~s}, 12 \mathrm{H}) \mathrm{ppm} ;{ }^{13} \mathrm{C}\left\{{ }^{1} \mathrm{H}\right\} \mathrm{NMR}\left(\mathrm{CDCl}_{3}, 125.8\right.$ $\mathrm{MHz}): \delta 146.1,145.7,136.8,134.5,133.3,132.5,131.0,129.5,129.3$, 125.2, 120.4, 84.3, 25.0 ppm; ${ }^{11} \mathrm{~B}$ NMR $\left(\mathrm{CDCl}_{3}, 128.4 \mathrm{MHz}\right): \delta 30.9$ (br, 2B) ppm; IR: $\nu=3352,2971,1602,1359,1307,1141,1125,778$, $760 \mathrm{~cm}^{-1}$; HRMS (ESI) $\mathrm{m} / z$ calcd for $\mathrm{C}_{20} \mathrm{H}_{23} \mathrm{~B}_{2} \mathrm{NO}_{2} \mathrm{Cl}[\mathrm{M}+\mathrm{H}]^{+}$ 366.1603 , found 366.1608 .

2-(Benzothiophen-2-yl)-8-(4,4,5,5-tetramethyl-1,3,2-dioxaboryl)2,1-borazaronaphthalene (3l). Reaction was run at $80^{\circ} \mathrm{C}$, and the product was obtained as a white solid $(117 \mathrm{mg}, 60 \%, 0.5 \mathrm{mmol} \mathrm{scale})$; mp: $163-164{ }^{\circ} \mathrm{C} ;{ }^{1} \mathrm{H}$ NMR $\left(\mathrm{CDCl}_{3}, 500.4 \mathrm{MHz}\right): \delta 10.52(\mathrm{~s}, 1 \mathrm{H})$, $8.16(\mathrm{~d}, J=11.5 \mathrm{~Hz}, 1 \mathrm{H}), 8.03-7.96(\mathrm{~m}, 3 \mathrm{H}), 7.95-7.90(\mathrm{~m}, 1 \mathrm{H})$, $7.79(\mathrm{~d}, J=7.4 \mathrm{~Hz}, 1 \mathrm{H}), 7.43-7.36(\mathrm{~m}, 2 \mathrm{H}), 7.30(\mathrm{dd}, J=11.5,1.9$ $\mathrm{Hz}, 1 \mathrm{H}), 7.23(\mathrm{t}, J=7.4 \mathrm{~Hz}, 1 \mathrm{H}), 1.53(\mathrm{~s}, 12 \mathrm{H}) \mathrm{ppm} ;{ }^{13} \mathrm{C}\left\{{ }^{1} \mathrm{H}\right\} \mathrm{NMR}$ $\left(\mathrm{CDCl}_{3}, 125.8 \mathrm{MHz}\right): \delta 145.8,145.7,143.1,141.5,136.9,133.4,131.0$, $125.4,124.6,124.0,123.9,122.5,120.4,84.3,25.0 \mathrm{ppm} ;{ }^{11} \mathrm{~B}$ NMR $\left(\mathrm{CDCl}_{3}, 128.4 \mathrm{MHz}\right): \delta 30.5$ (br, 2B) ppm; IR: $\nu=3335,2976,1597$, $1353,1307,1137,798 \mathrm{~cm}^{-1}$; HRMS (ESI) $\mathrm{m} / z$ calcd for $\mathrm{C}_{22} \mathrm{H}_{24} \mathrm{~B}_{2} \mathrm{NO}_{2} \mathrm{~S}[\mathrm{M}+\mathrm{H}]^{+}$388.1714, found 388.1715.

2-(Furan-3-yl)-8-(4,4,5,5-tetramethyl-1,3,2-dioxaboryl)-2,1-borazaronaphthalene $(3 \mathrm{~m})$. Reaction was run at $80{ }^{\circ} \mathrm{C}$, and the product was obtained as a white solid (33 mg, $21 \%, 0.5 \mathrm{mmol} \mathrm{scale})$; mp: 195$197{ }^{\circ} \mathrm{C}$; ${ }^{1} \mathrm{H} \mathrm{NMR}\left(\mathrm{CDCl}_{3}, 500.4 \mathrm{MHz}\right): \delta 10.44(\mathrm{~s}, 1 \mathrm{H}), 8.13$ (d, $J=$ $11.4 \mathrm{~Hz}, 1 \mathrm{H}), 7.80(\mathrm{~s}, 1 \mathrm{H}), 7.66(\mathrm{~d}, J=7.8 \mathrm{~Hz}, 1 \mathrm{H}), 7.45(\mathrm{t}, J=7.6$ $\mathrm{Hz}, 1 \mathrm{H}), 7.30(\mathrm{~d}, J=8.1 \mathrm{~Hz}, 1 \mathrm{H}), 7.24-7.14(\mathrm{~m}, 2 \mathrm{H}), 7.03(\mathrm{~s}, 1 \mathrm{H})$, $1.51(\mathrm{~s}, 12 \mathrm{H}) \mathrm{ppm} ;{ }^{13} \mathrm{C}\left\{{ }^{1} \mathrm{H}\right\} \mathrm{NMR}\left(\mathrm{CDCl}_{3}, 125.8 \mathrm{MHz}\right): \delta$ 147.1, 145.0, 140.5, 129.3, 128.1, 125.5, 120.5, 118.0, 116.0, 84.8, 24.9 ppm; ${ }^{11} \mathrm{~B}$ NMR $\left(\mathrm{CDCl}_{3}, 128.4 \mathrm{MHz}\right): \delta 27.4$ (br, 2B) ppm; IR: $\nu=3348$, 2971, 1574, 1560, 1451, 1303, 1138, $778 \mathrm{~cm}^{-1}$; HRMS (ESI) $\mathrm{m} / z$ calcd for $\mathrm{C}_{18} \mathrm{H}_{22} \mathrm{~B}_{2} \mathrm{NO}_{3}[\mathrm{M}+\mathrm{H}]^{+} 322.1786$, found 322.1793 .

2-Methyl-8-(4,4,5,5-tetramethyl-1,3,2-dioxaboryl)-2,1-borazaronaphthalene (3n). Reaction was run at $80{ }^{\circ} \mathrm{C}$, and the product was obtained as a thick yellow oil ( $80 \mathrm{mg}, 60 \%, 0.5 \mathrm{mmol}$ scale $) ;{ }^{1} \mathrm{H}$ NMR $\left(\mathrm{CDCl}_{3}, 500.4 \mathrm{MHz}\right): \delta 9.68(\mathrm{~s}, 1 \mathrm{H}), 7.92(\mathrm{~d}, J=9.5 \mathrm{~Hz}, 2 \mathrm{H}), 7.70(\mathrm{~d}$, $J=7.8 \mathrm{~Hz}, 1 \mathrm{H}), 7.13(\mathrm{t}, J=7.7 \mathrm{~Hz}, 1 \mathrm{H}), 6.80(\mathrm{~d}, J=11.4 \mathrm{~Hz}, 1 \mathrm{H})$, $1.44(\mathrm{~s}, 12 \mathrm{H}), 0.79(\mathrm{~s}, 3 \mathrm{H}) \mathrm{ppm} ;{ }^{13} \mathrm{C}\left\{{ }^{1} \mathrm{H}\right\}$ NMR $\left(\mathrm{CDCl}_{3}, 125.8\right.$ $\mathrm{MHz}): \delta 145.8,144.1,136.3,133.2,124.6,119.5,83.9,24.9 \mathrm{ppm} ;{ }^{1{ }^{1} \mathrm{~B}}$ NMR $\left(\mathrm{CDCl}_{3}, 128.4 \mathrm{MHz}\right): \delta 36.5,30.0$ ppm; IR: $\nu=3358,2984$, $1601,1354,1311,1136,1101,760 \mathrm{~cm}^{-1}$; HRMS (ESI) $\mathrm{m} / z$ calcd for $\mathrm{C}_{15} \mathrm{H}_{22} \mathrm{~B}_{2} \mathrm{NO}_{2}[\mathrm{M}+\mathrm{H}]^{+} 270.1837$, found 270.1827 .

2-Hexyl-8-(4,4,5,5-tetramethyl-1,3,2-dioxaboryl)-2,1-borazaronaphthalene (3o). Reaction was run at $40{ }^{\circ} \mathrm{C}$, and the product was obtained as a white solid (65 mg, 91\%, $0.21 \mathrm{mmol} \mathrm{scale})$; $\mathrm{mp}: 127-$ $130{ }^{\circ} \mathrm{C} ;{ }^{1} \mathrm{H}$ NMR $\left(\mathrm{CDCl}_{3}, 500.4 \mathrm{MHz}\right): \delta 9.85(\mathrm{~s}, 1 \mathrm{H}), 7.98-7.92$ $(\mathrm{m}, 2 \mathrm{H}), 7.76-7.68(\mathrm{~m}, 1 \mathrm{H}), 7.15(\mathrm{t}, J=7.4 \mathrm{~Hz}, 1 \mathrm{H}), 6.82(\mathrm{~d}, J=11.4$ $\mathrm{Hz}, 1 \mathrm{H}), 1.73-1.65(\mathrm{~m}, 2 \mathrm{H}), 1.48-1.43(\mathrm{~m}, 14 \mathrm{H}), 1.42-1.33(\mathrm{~m}$, $6 \mathrm{H}), 0.95(\mathrm{t}, J=6.7 \mathrm{~Hz}, 3 \mathrm{H}) \mathrm{ppm} ;{ }^{13} \mathrm{C}\left\{{ }^{1} \mathrm{H}\right\} \mathrm{NMR}\left(\mathrm{CDCl}_{3}, 125.8\right.$ $\mathrm{MHz}): \delta 145.9,144.0,136.2,133.2,124.8,119.6,83.9,32.3,32.0,25.4$, 24.9, 22.6, $14.1 \mathrm{ppm} ;{ }^{11} \mathrm{~B}$ NMR $\left(\mathrm{CDCl}_{3}, 128.4 \mathrm{MHz}\right): \delta 37.5,29.8$ ppm; IR: $\nu=3354,2921,1600,1354,1303,1137,762 \mathrm{~cm}^{-1}$; HRMS (ESI) $m / z$ calcd for $\mathrm{C}_{20} \mathrm{H}_{32} \mathrm{~B}_{2} \mathrm{NO}_{2}[\mathrm{M}+\mathrm{H}]^{+} 340.2623$, found 340.2630 .

2-Phenethyl-8-(4,4,5,5-tetramethyl-1,3,2-dioxaboryl)-2,1-borazaronaphthalene (3p). Reaction was run at $40^{\circ} \mathrm{C}$, and the product was obtained as a white solid (111 mg, $62 \%, 0.5 \mathrm{mmol} \mathrm{scale})$; mp: $58-$ $60{ }^{\circ} \mathrm{C} ;{ }^{1} \mathrm{H}$ NMR $\left(\mathrm{CDCl}_{3}, 500.4 \mathrm{MHz}\right): \delta 9.91(\mathrm{~s}, 1 \mathrm{H}), 8.00-7.91(\mathrm{~m}$, $2 \mathrm{H}), 7.72(\mathrm{~d}, J=7.7 \mathrm{~Hz}, 1 \mathrm{H}), 7.36-7.29(\mathrm{~m}, 4 \mathrm{H}), 7.23-7.11(\mathrm{~m}$, $2 \mathrm{H}), 6.84(\mathrm{dd}, J=11.5,1.9 \mathrm{~Hz}, 1 \mathrm{H}), 3.01(\mathrm{t}, J=8.3 \mathrm{~Hz}, 2 \mathrm{H}), 1.71(\mathrm{t}, J$ 
$=8.3 \mathrm{~Hz}, 2 \mathrm{H}), 1.43(\mathrm{~s}, 12 \mathrm{H}) \mathrm{ppm} ;{ }^{13} \mathrm{C}\left\{{ }^{1} \mathrm{H}\right\}$ NMR $\left(\mathrm{CDCl}_{3}, 125.8\right.$ $\mathrm{MHz}): \delta 145.7,145.2,144.4,136.4,133.3,128.2,128.0,127.9,125.3$, 124.8, 119.8, 84.0, 31.5, 24.9 ppm; ${ }^{11} \mathrm{~B}$ NMR $\left(\mathrm{CDCl}_{3}, 128.4 \mathrm{MHz}\right)$ : $\delta 37.8,31.5$ ppm; IR: $\nu=3349,2978,1598,1355,1311,1137,1127$, $759 \mathrm{~cm}^{-1}$; HRMS (ESI) $\mathrm{m} / z$ calcd for $\mathrm{C}_{22} \mathrm{H}_{28} \mathrm{~B}_{2} \mathrm{NO}_{2}[\mathrm{M}+\mathrm{H}]^{+}$ 360.2306 , found 360.2305 .

2-Cyclopropyl-8-(4,4,5,5-tetramethyl-1,3,2-dioxaboryl)-2,1-borazaronaphthalene $(3 q)$. Reaction was run at $80{ }^{\circ} \mathrm{C}$, and the product was obtained as a white solid ( $115 \mathrm{mg}, 78 \%, 0.5 \mathrm{mmol} \mathrm{scale})$; mp: $75-$ $76{ }^{\circ} \mathrm{C} ;{ }^{1} \mathrm{H}$ NMR $\left(\mathrm{CDCl}_{3}, 500.4 \mathrm{MHz}\right): \delta 9.61(\mathrm{~s}, 1 \mathrm{H}), 8.04-7.84(\mathrm{~m}$, $2 \mathrm{H}), 7.69(\mathrm{dd}, J=7.7,1.6 \mathrm{~Hz}, 1 \mathrm{H}), 7.13(\mathrm{t}, J=7.4 \mathrm{~Hz}, 1 \mathrm{H}), 6.62(\mathrm{dd}$, $J=11.6,1.9 \mathrm{~Hz}, 1 \mathrm{H}), 1.45(\mathrm{~s}, 12 \mathrm{H}), 0.97-0.80(\mathrm{~m}, 2 \mathrm{H}), 0.72-0.57$ $(\mathrm{m}, 2 \mathrm{H}), 0.37(\mathrm{tt}, J=8.8,6.0 \mathrm{~Hz}, 1 \mathrm{H}) \mathrm{ppm} ;{ }^{13} \mathrm{C}\left\{{ }^{1} \mathrm{H}\right\} \operatorname{NMR}\left(\mathrm{CDCl}_{3}\right.$, $125.8 \mathrm{MHz}): \delta 145.9,144.5,136.4,133.2,124.7,119.4,83.9,24.9,6.3$ ppm; ${ }^{11} \mathrm{~B} \mathrm{NMR}\left(\mathrm{CDCl}_{3}, 128.4 \mathrm{MHz}\right): \delta 37.3,30.0 \mathrm{ppm}$; IR: $\nu=3356$, 2979, 1598, 1353, 1297, 1135, 1109, 794, $774 \mathrm{~cm}^{-1}$; HRMS (ESI) $\mathrm{m} / z$ calcd for $\mathrm{C}_{17} \mathrm{H}_{24} \mathrm{~B}_{2} \mathrm{NO}_{2}[\mathrm{M}+\mathrm{H}]^{+} 296.1993$, found 296.1990.

2-Cyclohexyl-8-(4,4,5,5-tetramethyl-1,3,2-dioxaboryl)-2,1-borazaronaphthalene (3r). Reaction was run at $40{ }^{\circ} \mathrm{C}$, and the product was obtained as a yellow solid (138 mg, $82 \%, 0.5 \mathrm{mmol} \mathrm{scale}) ; \mathrm{mp}$ : 69-70 ${ }^{\circ} \mathrm{C} ;{ }^{1} \mathrm{H} \mathrm{NMR}\left(\mathrm{CDCl}_{3}, 500.4 \mathrm{MHz}\right): \delta 9.94(\mathrm{~s}, 1 \mathrm{H}), 8.07-7.87$ (m, 2H), $7.72(\mathrm{~d}, J=7.3 \mathrm{~Hz}, 1 \mathrm{H}), 7.15(\mathrm{t}, J=7.4 \mathrm{~Hz}, 1 \mathrm{H}), 6.88(\mathrm{dd}, J$ $=11.5,1.9 \mathrm{~Hz}, 1 \mathrm{H}), 2.08-1.97(\mathrm{~m}, 2 \mathrm{H}), 1.85-1.71(\mathrm{~m}, 3 \mathrm{H}), 1.54-$ $1.38(\mathrm{~m}, 18 \mathrm{H}) \mathrm{ppm} ;{ }^{13} \mathrm{C}\left\{{ }^{1} \mathrm{H}\right\}$ NMR $\left(\mathrm{CDCl}_{3}, 125.8 \mathrm{MHz}\right): \delta 145.7$, $144.4,136.2,133.2,124.8,119.6,83.9,29.6,27.7,27.2,24.9 \mathrm{ppm} ;{ }^{11} \mathrm{~B}$ NMR $\left(\mathrm{CDCl}_{3}, 128.4 \mathrm{MHz}\right): \delta 40.2,31.8 \mathrm{ppm}$; IR: $\nu=3356,2917$, $1599,1356,1309,1137,1124,800 \mathrm{~cm}^{-1}$; HRMS (EI) $\mathrm{m} / z$ calcd for $\mathrm{C}_{20} \mathrm{H}_{29} \mathrm{~B}_{2} \mathrm{NO}_{2}[\mathrm{M}+\mathrm{Na}]^{+}$337.2384, found 337.2386.

3-Bromo-2-phenyl-8-(4,4,5,5-tetramethyl-1,3,2-dioxaboryl)-2,1borazaronaphthalene $(4 a)$. Reaction was run at $80^{\circ} \mathrm{C}$, and the product was obtained as a white solid ( $130 \mathrm{mg}, 64 \%, 0.5 \mathrm{mmol} \mathrm{scale})$; mp: $72-74{ }^{\circ} \mathrm{C} ;{ }^{1} \mathrm{H}$ NMR $\left(\mathrm{CDCl}_{3}, 500.4 \mathrm{MHz}\right): \delta 10.48(\mathrm{~s}, 1 \mathrm{H}), 8.49$ $(\mathrm{s}, 1 \mathrm{H}), 8.22-8.14(\mathrm{~m}, 2 \mathrm{H}), 8.06(\mathrm{dd}, J=7.1,1.5 \mathrm{~Hz}, 1 \mathrm{H}), 7.75(\mathrm{~d}, J=$ $7.8 \mathrm{~Hz}, 1 \mathrm{H}), 7.64-7.50(\mathrm{~m}, 3 \mathrm{H}), 7.27(\mathrm{t}, J=7.5 \mathrm{~Hz}, 1 \mathrm{H}), 1.47(\mathrm{~s}$, $12 \mathrm{H}) \mathrm{ppm} ;{ }^{13} \mathrm{C}\left\{{ }^{1} \mathrm{H}\right\}$ NMR $\left(\mathrm{CDCl}_{3}, 125.8 \mathrm{MHz}\right): \delta 147.1,144.9$, $137.1,133.6,132.5,129.3,127.8,124.7,121.0,84.4,25.0 \mathrm{ppm} ;{ }^{11} \mathrm{~B}$ NMR $\left(\mathrm{CDCl}_{3}, 128.4 \mathrm{MHz}\right): \delta 30.6(\mathrm{br}, 2 \mathrm{~B})$ ppm; IR: $\nu=3338,2974$, 1597, 1373, 1344, 1309, 1132, $698 \mathrm{~cm}^{-1}$; HRMS (EI) $\mathrm{m} / z$ calcd for $\mathrm{C}_{20} \mathrm{H}_{22} \mathrm{~B}_{2} \mathrm{NO}_{2} \mathrm{Br}[\mathrm{M}]^{+}$409.1020, found 409.1041.

3-Bromo-8-(4,4,5,5-tetramethyl-1,3,2-dioxaboryl)-2-(4(trifluoromethyl)phenyl)-2,1-borazaronaphthalene (4b). Reaction was run at $80{ }^{\circ} \mathrm{C}$, and the product was obtained as an off-white solid (169 mg, 71\%, $0.5 \mathrm{mmol}$ scale); mp: $122-124{ }^{\circ} \mathrm{C}$; ${ }^{1} \mathrm{H}$ NMR $\left(\mathrm{CDCl}_{3}, 500.4 \mathrm{MHz}\right): \delta 10.50(\mathrm{~s}, 1 \mathrm{H}), 8.46(\mathrm{~s}, 1 \mathrm{H}), 8.20(\mathrm{~d}, J=7.4$ $\mathrm{Hz}, 2 \mathrm{H}), 8.08(\mathrm{~d}, J=7.0 \mathrm{~Hz}, 1 \mathrm{H}), 7.79(\mathrm{~d}, J=7.5 \mathrm{~Hz}, 2 \mathrm{H}), 7.73(\mathrm{~d}, J$ $=7.6 \mathrm{~Hz}, 1 \mathrm{H}), 7.28(\mathrm{t}, J=7.1 \mathrm{~Hz}, 1 \mathrm{H}), 1.47(\mathrm{~s}, 12 \mathrm{H}) \mathrm{ppm} ;{ }^{13} \mathrm{C}\left\{{ }^{1} \mathrm{H}\right\}$ NMR $\left(\mathrm{CDCl}_{3}, 125.8 \mathrm{MHz}\right): \delta$ 147.4, 144.6, 137.4, 133.7, 132.5, 131.0 $(\mathrm{q}, J=32.1 \mathrm{~Hz}), 124.8,124.4(\mathrm{q}, J=271.8 \mathrm{~Hz}), 124.3(\mathrm{q}, J=3.9 \mathrm{~Hz})$, 121.4, 84.5, 24.9 ppm; ${ }^{19} \mathrm{~F}\left\{{ }^{1} \mathrm{H}\right\}$ NMR $\left(\mathrm{CDCl}_{3}, 470.8 \mathrm{MHz}\right): \delta-62.6$ ppm; ${ }^{11} \mathrm{~B}$ NMR $\left(\mathrm{CDCl}_{3}, 128.4 \mathrm{MHz}\right): \delta 32.0$ (br, 2B) ppm; IR: $\nu=$ 3340, 2980, 1598, 1558, 1320, 1120, 1068, $761 \mathrm{~cm}^{-1}$; HRMS (EI) $\mathrm{m} / z$ calcd for $\mathrm{C}_{21} \mathrm{H}_{21} \mathrm{~B}_{2} \mathrm{NO}_{2} \mathrm{~F}_{3} \mathrm{Br}[\mathrm{M}]^{+}$477.0894, found 477.0891.

3,6-Dibromo-2-phenyl-8-(4,4,5,5-tetramethyl-1,3,2-dioxaboryl)2,1-borazaronaphthalene (4c). Reaction was run at $80{ }^{\circ} \mathrm{C}$, and the product was obtained as a white solid ( $52 \mathrm{mg}, 43 \%, 0.25 \mathrm{mmol} \mathrm{scale})$; mp: $136-138{ }^{\circ} \mathrm{C} ;{ }^{1} \mathrm{H}$ NMR $\left(\mathrm{CDCl}_{3}, 500.4 \mathrm{MHz}\right): \delta 10.34(\mathrm{~s}, 1 \mathrm{H})$, $8.35(\mathrm{~s}, 1 \mathrm{H}), 8.13-8.03(\mathrm{~m}, 3 \mathrm{H}), 7.82(\mathrm{~s}, 1 \mathrm{H}), 7.54-7.47(\mathrm{~m}, 3 \mathrm{H})$, $1.44(\mathrm{~s}, 12 \mathrm{H}) \mathrm{ppm} ;{ }^{13} \mathrm{C}\left\{{ }^{1} \mathrm{H}\right\}$ NMR $\left(\mathrm{CDCl}_{3}, 125.8 \mathrm{MHz}\right): \delta 145.8$, 143.4, 139.2, 134.0, 133.5, 129.5, 127.8, 126.3, 113.8, 84.8, 24.9 ppm; ${ }^{11} \mathrm{~B}$ NMR $\left(\mathrm{CDCl}_{3}, 128.4 \mathrm{MHz}\right): \delta 32.7,30.7 \mathrm{ppm}$; IR: $\nu=3550,2975$, $1593,1555,1416,1370,1312,1141,848,793 \mathrm{~cm}^{-1}$; HRMS (EI) $\mathrm{m} / z$ calcd for $\mathrm{C}_{20} \mathrm{H}_{21} \mathrm{~B}_{2} \mathrm{NO}_{2} \mathrm{Br}_{2}[\mathrm{M}]^{+}$487.0125, found 487.0122.

3-(4-Fluorophenyl)-2-phenyl-8-(4,4,5,5-tetramethyl-1,3,2-dioxaboryl)-2,1-borazaronaphthalene $(4 d)$. Reaction was run at $60{ }^{\circ} \mathrm{C}$, and the product was obtained as a light brown solid (45 mg, 42\%, 0.25 mmol scale); mp: $164-165{ }^{\circ} \mathrm{C}$; ${ }^{1} \mathrm{H}$ NMR $\left(\mathrm{CDCl}_{3}, 500.4 \mathrm{MHz}\right): \delta$ $10.39(\mathrm{~s}, 1 \mathrm{H}), 8.03-7.96(\mathrm{~m}, 2 \mathrm{H}), 7.83(\mathrm{~d}, J=7.1 \mathrm{~Hz}, 1 \mathrm{H}), 7.57(\mathrm{~d}, J$ $=7.9 \mathrm{~Hz}, 2 \mathrm{H}), 7.39-7.29(\mathrm{~m}, 5 \mathrm{H}), 7.24(\mathrm{t}, J=7.4 \mathrm{~Hz}, 1 \mathrm{H}), 7.03(\mathrm{t}, J$ $=8.8 \mathrm{~Hz}, 2 \mathrm{H}), 1.46(\mathrm{~s}, 12 \mathrm{H}) \mathrm{ppm} ;{ }^{13} \mathrm{C}\left\{{ }^{1} \mathrm{H}\right\} \operatorname{NMR}\left(\mathrm{CDCl}_{3}, 125.8\right.$ $\mathrm{MHz}): \delta 161.7(\mathrm{~d}, J=244.2 \mathrm{~Hz}), 145.2,143.7,140.5,136.7,133.6$,
$133.4,130.1$ (d, $J=7.8 \mathrm{~Hz}), 128.6,127.7,124.7,120.6,114.7$ (d, $J=$ $21.1 \mathrm{~Hz}), 84.2,24.9 \mathrm{ppm} ;{ }^{19} \mathrm{~F}\left\{{ }^{1} \mathrm{H}\right\} \mathrm{NMR}\left(\mathrm{CDCl}_{3}, 470.8 \mathrm{MHz}\right): \delta$ -111.7 ppm; ${ }^{11} \mathrm{~B}$ NMR $\left(\mathrm{CDCl}_{3}, 128.4 \mathrm{MHz}\right): \delta 34.0,31.2 \mathrm{ppm}$; IR: $\nu$ $=3348,2970,2915,1600,1446,1346,1310,1131,760 \mathrm{~cm}^{-1}$; HRMS (EI) $\mathrm{m} / z$ calcd for $\mathrm{C}_{26} \mathrm{H}_{26} \mathrm{~B}_{2} \mathrm{NO}_{2} \mathrm{~F}[\mathrm{M}]^{+}$425.2134, found 425.2144.

7-Fluoro-8-(4,4,5,5-tetramethyl-1,3,2-dioxaboryl)-2-(4(trifluoromethyl)phenyl)-2,1-borazaronaphthalene (4e). Reaction was run at $80{ }^{\circ} \mathrm{C}$, and the product was obtained as brown cystals (98 mg, 47\%, $0.5 \mathrm{mmol} \mathrm{scale})$; $\mathrm{mp}: 132-135{ }^{\circ} \mathrm{C} ;{ }^{1} \mathrm{H}$ NMR $\left(\mathrm{CDCl}_{3}\right.$, $500.4 \mathrm{MHz}): \delta 10.80(\mathrm{~s}, 1 \mathrm{H}), 8.15(\mathrm{~d}, J=11.5 \mathrm{~Hz}, 1 \mathrm{H}), 8.08(\mathrm{~d}, J=$ $7.8 \mathrm{~Hz}, 2 \mathrm{H}), 7.79-7.70(\mathrm{~m}, 3 \mathrm{H}), 7.23(\mathrm{dd}, J=11.5,1.4 \mathrm{~Hz}, 1 \mathrm{H}), 6.95$ $(\mathrm{t}, J=9.0 \mathrm{~Hz}, 1 \mathrm{H}), 1.51(\mathrm{~s}, 12 \mathrm{H}) \mathrm{ppm} ;{ }^{13} \mathrm{C}\left\{{ }^{1} \mathrm{H}\right\} \mathrm{NMR}\left(\mathrm{CDCl}_{3}, 125.8\right.$ $\mathrm{MHz}): \delta 168.6(\mathrm{~d}, J=254.2 \mathrm{~Hz}), 146.6(\mathrm{~d}, J=11.2 \mathrm{~Hz}), 145.9,135.14$ $(\mathrm{d}, J=11.7 \mathrm{~Hz}), 132.9,131.2(\mathrm{q}, J=32.2 \mathrm{~Hz}), 124.7(\mathrm{q}, J=3.7 \mathrm{~Hz})$, $124.3(\mathrm{q}, J=272.2 \mathrm{~Hz}), 122.2,109.5(\mathrm{~d}, J=26.8 \mathrm{~Hz}), 84.1,24.9 \mathrm{ppm}$; ${ }^{19} \mathrm{~F}\left\{{ }^{1} \mathrm{H}\right\}$ NMR $\left(\mathrm{CDCl}_{3}, 470.8 \mathrm{MHz}\right): \delta-62.7,-97.1 \mathrm{ppm} ;{ }^{11} \mathrm{~B}$ NMR $\left(\mathrm{CDCl}_{3}, 128.4 \mathrm{MHz}\right): \delta 33.6,30.2 \mathrm{ppm}$; IR: $\nu=3345,2982,1599$, $1320,1208,1162,1138,1119,1066,802 \mathrm{~cm}^{-1}$; HRMS (EI) $\mathrm{m} / z$ calcd for $\mathrm{C}_{21} \mathrm{H}_{21} \mathrm{~B}_{2} \mathrm{NO}_{2} \mathrm{~F}_{4}[\mathrm{M}]^{+}$417.1695, found 417.1695.

6-Cyano-8-(4,4,5,5-tetramethyl-1,3,2-dioxaboryl)-2-(4(trifluoromethyl)phenyl)-2,1-borazaronaphthalene (4f). Reaction was run at $80{ }^{\circ} \mathrm{C}$ for $24 \mathrm{~h}$, and the product was obtained as a white solid (202 mg, 93\%, $1.0 \mathrm{mmol}$ scale); mp: $195-196{ }^{\circ} \mathrm{C}$; ${ }^{1} \mathrm{H}$ NMR $\left(\mathrm{CDCl}_{3}, 500.4 \mathrm{MHz}\right): \delta 10.66(\mathrm{~s}, 1 \mathrm{H}), 8.22(\mathrm{~d}, J=1.6 \mathrm{~Hz}, 1 \mathrm{H}), 8.15$ (d, $J=11.6 \mathrm{~Hz}, 1 \mathrm{H}), 8.12-8.05(\mathrm{~m}, 3 \mathrm{H}), 7.76(\mathrm{~d}, J=7.9 \mathrm{~Hz}, 2 \mathrm{H})$, $7.41(\mathrm{dd}, J=11.7,1.2 \mathrm{~Hz}, 1 \mathrm{H}), 1.49(\mathrm{~s}, 12 \mathrm{H}) \mathrm{ppm} ;{ }^{13} \mathrm{C}\left\{{ }^{1} \mathrm{H}\right\} \mathrm{NMR}$ $\left(\mathrm{CDCl}_{3}, 125.8 \mathrm{MHz}\right): \delta 147.7,145.4,139.2,137.3,133.1,131.8$ (q, $J=$ $32.0 \mathrm{~Hz}), 125.2,124.9(\mathrm{q}, J=3.7 \mathrm{~Hz}), 124.1(\mathrm{q}, J=271.4, \mathrm{~Hz}), 119.0$, 104.2, 85.0, $25.0 \mathrm{ppm} ;{ }^{19} \mathrm{~F}\left\{{ }^{1} \mathrm{H}\right\} \mathrm{NMR}\left(\mathrm{CDCl}_{3}, 470.8 \mathrm{MHz}\right): \delta-62.9$ ppm; ${ }^{11} \mathrm{~B}$ NMR $\left(\mathrm{CDCl}_{3}, 128.4 \mathrm{MHz}\right): \delta 33.7,30.3 \mathrm{ppm}$; IR: $\nu=3346$, 2985, 2928, 1719, 1608, 1395, 1317, 1135, 1118, $792 \mathrm{~cm}^{-1}$; HRMS (EI) $m / z$ calcd for $\mathrm{C}_{22} \mathrm{H}_{21} \mathrm{~B}_{2} \mathrm{~N}_{2} \mathrm{O}_{2} \mathrm{~F}_{3}[\mathrm{M}]^{+} 424.1741$, found 424.1750 .

8-(4,4,5,5-Tetramethyl-1,3,2-dioxaboryl)-6-(trifluoromethyl)-2-(4trifluoromethyl)phenyl)-2,1-borazaronaphthalene (4g). Reaction was run at $80{ }^{\circ} \mathrm{C}$, and the product was obtained as a white solid (140 mg, 82\%, $0.5 \mathrm{mmol} \mathrm{scale})$; $\mathrm{mp}: 160-162{ }^{\circ} \mathrm{C} ;{ }^{1} \mathrm{H} \mathrm{NMR}\left(\mathrm{CDCl}_{3}\right.$, $500.4 \mathrm{MHz}): \delta 10.66(\mathrm{~s}, 1 \mathrm{H}), 8.23(\mathrm{~d}, J=1.3 \mathrm{~Hz}, 1 \mathrm{H}), 8.20(\mathrm{~d}, J=$ $11.6 \mathrm{~Hz}, 1 \mathrm{H}), 8.09(\mathrm{~d}, J=7.8 \mathrm{~Hz}, 2 \mathrm{H}), 8.05(\mathrm{~s}, 1 \mathrm{H}), 7.77(\mathrm{~d}, J=7.8$ $\mathrm{Hz}, 2 \mathrm{H}), 7.39(\mathrm{dd}, J=11.6,1.5 \mathrm{~Hz}, 1 \mathrm{H}), 1.51(\mathrm{~s}, 12 \mathrm{H}) \mathrm{ppm} ;{ }^{13} \mathrm{C}\left\{{ }^{1} \mathrm{H}\right\}$ NMR $\left(\mathrm{CDCl}_{3}, 125.8 \mathrm{MHz}\right): \delta 147.3,146.0,133.0,133.0(\mathrm{q}, J=3.7$ $\mathrm{Hz}), 131.5(\mathrm{q}, J=32.1 \mathrm{~Hz}), 130.3(\mathrm{q}, J=3.8 \mathrm{~Hz}), 124.8(\mathrm{q}, J=3.8$ $\mathrm{Hz}), 124.7,124.4(\mathrm{q}, J=272.2 \mathrm{~Hz}), 124.2(\mathrm{q}, J=271.8 \mathrm{~Hz}), 122.8(\mathrm{q}$, $J=32.7 \mathrm{~Hz}), 84.8,25.0 \mathrm{ppm} ;{ }^{19} \mathrm{~F}\left\{{ }^{1} \mathrm{H}\right\} \mathrm{NMR}\left(\mathrm{CDCl}_{3}, 470.8 \mathrm{MHz}\right): \delta$ $-61.3,-62.8 \mathrm{ppm} ;{ }^{11} \mathrm{~B} \mathrm{NMR}\left(\mathrm{CDCl}_{3}, 128.4 \mathrm{MHz}\right): \delta 33.5,31.0 \mathrm{ppm}$; IR: $\nu=3350,2985,1618,1581,1319,1142,1113,1067,793 \mathrm{~cm}^{-1}$; HRMS (EI) $m / z$ calcd for $\mathrm{C}_{22} \mathrm{H}_{21} \mathrm{~B}_{2} \mathrm{NO}_{2} \mathrm{~F}_{6}[\mathrm{M}]^{+}$467.1663, found 467.1652 .

6-Isopropyl-8-(4,4,5,5-tetramethyl-1,3,2-dioxaboryl)-2-(4(trifluoromethyl)phenyl)-2,1-borazaronaphthalene (4h). Reaction was run at $80{ }^{\circ} \mathrm{C}$, and the product was obtained as a white solid (174 mg, 79\%, $0.5 \mathrm{mmol} \mathrm{scale})$; mp: $198-202{ }^{\circ} \mathrm{C} ;{ }^{1} \mathrm{H}$ NMR ( $\mathrm{CDCl}_{3}$, $500.4 \mathrm{MHz}): \delta 10.54(\mathrm{~s}, 1 \mathrm{H}), 8.18(\mathrm{~d}, J=11.5 \mathrm{~Hz}, 1 \mathrm{H}), 8.09(\mathrm{~d}, J=$ $8.0 \mathrm{~Hz}, 2 \mathrm{H}), 7.90(\mathrm{~d}, J=2.1 \mathrm{~Hz}, 1 \mathrm{H}), 7.74(\mathrm{~d}, J=8.0 \mathrm{~Hz}, 2 \mathrm{H}), 7.65$ $(\mathrm{d}, J=2.0 \mathrm{~Hz}, 1 \mathrm{H}), 7.28(\mathrm{dd}, J=11.4,2.1 \mathrm{~Hz}, 1 \mathrm{H}), 3.06$ (sept, $J=6.9$ $\mathrm{Hz}, 1 \mathrm{H}), 1.49(\mathrm{~s}, 12 \mathrm{H}), 1.35(\mathrm{~d}, J=6.9 \mathrm{~Hz}, 6 \mathrm{H}) \mathrm{ppm} ;{ }^{13} \mathrm{C}\left\{{ }^{1} \mathrm{H}\right\} \mathrm{NMR}$ $\left(\mathrm{CDCl}_{3}, 125.8 \mathrm{MHz}\right): \delta 146.2,144.0,140.9,135.9,132.9,130.9(\mathrm{q}, J=$ $32.2 \mathrm{~Hz}), 130.5,125.4,124.7(\mathrm{q}, J=3.8 \mathrm{~Hz}), 124.4(\mathrm{q}, J=272.1 \mathrm{~Hz})$, 84.2, 33.5, 25.0, $24.2 \mathrm{ppm} ;{ }^{19} \mathrm{~F}\left\{{ }^{1} \mathrm{H}\right\}$ NMR $\left(\mathrm{CDCl}_{3}, 470.8 \mathrm{MHz}\right): \delta$ -62.7 ppm; ${ }^{11} \mathrm{~B}$ NMR $\left(\mathrm{CDCl}_{3}, 128.4 \mathrm{MHz}\right): \delta 30.5$ (br, 2B) ppm; IR: $\nu=3350,2954,1572,1332,1163,1120,1067,789 \mathrm{~cm}^{-1}$; HRMS (EI) $m / z$ calcd for $\mathrm{C}_{24} \mathrm{H}_{28} \mathrm{~B}_{2} \mathrm{NO}_{2} \mathrm{~F}_{3}[\mathrm{M}]^{+}$441.2285, found 441.2253.

8-(4,4,5,5-Tetramethyl-1,3,2-dioxaboryl)-6-(trifluoromethoxy)-2(4-(trifluoromethyl)phenyl)-2,1-borazaronaphthalene (4i). Reaction was run at $80^{\circ} \mathrm{C}$, and the product was obtained as a white solid (171 $\mathrm{mg}, 71 \%, 0.5 \mathrm{mmol}$ scale); mp: $117-119^{\circ} \mathrm{C}$; ${ }^{1} \mathrm{H}$ NMR $\left(\mathrm{CDCl}_{3}, 500.4\right.$ $\mathrm{MHz}): \delta 10.57(\mathrm{~s}, 1 \mathrm{H}), 8.15(\mathrm{~d}, J=11.6 \mathrm{~Hz}, 1 \mathrm{H}), 8.08(\mathrm{~d}, J=7.8 \mathrm{~Hz}$, $2 \mathrm{H}), 7.85(\mathrm{~d}, J=2.4 \mathrm{~Hz}, 1 \mathrm{H}), 7.75(\mathrm{~d}, J=8.0 \mathrm{~Hz}, 2 \mathrm{H}), 7.64(\mathrm{~d}, J=2.0$ $\mathrm{Hz}, 1 \mathrm{H}), 7.37(\mathrm{dd}, J=11.6,1.8 \mathrm{~Hz}, 1 \mathrm{H}), 1.49(\mathrm{~s}, 12 \mathrm{H}) \mathrm{ppm} ;{ }^{13} \mathrm{C}\left\{{ }^{1} \mathrm{H}\right\}$ $\operatorname{NMR}\left(\mathrm{CDCl}_{3}, 125.8 \mathrm{MHz}\right): \delta 145.5,144.0,142.5,132.9,131.3(\mathrm{q}, J=$ $32.2 \mathrm{~Hz}), 129.6,126.0,124.8,124.8(\mathrm{q}, J=3.8 \mathrm{~Hz}), 124.2(\mathrm{q}, J=271.0$ 
$\mathrm{Hz}), 120.5(\mathrm{q}, J=256.2 \mathrm{~Hz}), 84.7,25.0(\mathrm{q}, J=79.7 \mathrm{~Hz}) \mathrm{ppm} ;{ }^{19} \mathrm{~F}$ $\left\{{ }^{1} \mathrm{H}\right\}$ NMR $\left(\mathrm{CDCl}_{3}, 470.8 \mathrm{MHz}\right): \delta-58.0,-62.8 \mathrm{ppm} ;{ }^{11} \mathrm{~B}$ NMR $\left(\mathrm{CDCl}_{3}, 128.4 \mathrm{MHz}\right): \delta 30.0$ (br, 2B) ppm; IR: $\nu=3353,2981,1574$, $1315,1259,1155,1140,1106,1067,774 \mathrm{~cm}^{-1}$; HRMS (EI) $\mathrm{m} / z$ calcd for $\mathrm{C}_{22} \mathrm{H}_{21} \mathrm{~B}_{2} \mathrm{NO}_{3} \mathrm{~F}_{6}[\mathrm{M}]^{+}$483.1612, found 483.1620 .

6, 8-Bis (4, 4, 5, 5-tetramethyl-1,3,2-dioxaboryl)-2-(4(trifluoromethyl)phenyl)-2,1-borazaronaphthalene (5a). Reaction was run at $80{ }^{\circ} \mathrm{C}$ with 3.0 equiv of $\mathrm{B}_{2} \mathrm{Pin}_{2}(381 \mathrm{mg}, 1.5 \mathrm{mmol})$, and the product was obtained as a white solid (203 $\mathrm{mg}, 78 \%, 0.5 \mathrm{mmol}$ scale); mp: $220-222{ }^{\circ} \mathrm{C} ;{ }^{1} \mathrm{H}$ NMR $\left(\mathrm{CDCl}_{3}, 500.4 \mathrm{MHz}\right): \delta 10.69$ (s, $1 \mathrm{H}), 8.49(\mathrm{~s}, 1 \mathrm{H}), 8.34(\mathrm{~s}, 1 \mathrm{H}), 8.24(\mathrm{dd}, J=11.6,2.9 \mathrm{~Hz}, 1 \mathrm{H}), 8.12$ $(\mathrm{d}, J=6.3 \mathrm{~Hz}, 2 \mathrm{H}), 7.77(\mathrm{~d}, J=6.5 \mathrm{~Hz}, 2 \mathrm{H}), 7.30(\mathrm{~d}, J=11.6 \mathrm{~Hz}$, $1 \mathrm{H}), 1.50(\mathrm{~s}, 12 \mathrm{H}), 1.43(\mathrm{~s}, 12 \mathrm{H}) \mathrm{ppm} ;{ }^{13} \mathrm{C}\left\{{ }^{1} \mathrm{H}\right\}$ NMR $\left(\mathrm{CDCl}_{3}\right.$, $125.8 \mathrm{MHz}): \delta 147.8,146.7,143.2,141.0,133.0(2 \times \mathrm{C}), 131.2(\mathrm{q}, J=$ $31.9 \mathrm{~Hz}), 124.7(\mathrm{q}, J=3.1 \mathrm{~Hz}), 124.4(\mathrm{q}, J=272.3 \mathrm{~Hz}), 84.2,83.7$, 25.0, $24.8 \mathrm{ppm} ;{ }^{19} \mathrm{~F}\left\{{ }^{1} \mathrm{H}\right\}$ NMR $\left(\mathrm{CDCl}_{3}, 470.8 \mathrm{MHz}\right): \delta-62.6 \mathrm{ppm}$; ${ }^{11} \mathrm{~B}$ NMR $\left(\mathrm{CDCl}_{3}, 128.4 \mathrm{MHz}\right): \delta 30.3(\mathrm{br}, 3 \mathrm{~B}) \mathrm{ppm}$; IR: $\nu=3352$, $2981,1605,1345,1321,1164,1138 \mathrm{~cm}^{-1}$; HRMS (ESI) $\mathrm{m} / z$ calcd for $\mathrm{C}_{27} \mathrm{H}_{34} \mathrm{~B}_{3} \mathrm{NO}_{4} \mathrm{~F}_{3}[\mathrm{M}+\mathrm{H}]^{+}$526.2719, found 526.2724.

2-Cyclopropyl-6,8-bis(4,4,5,5-tetramethyl-1,3,2-dioxaboryl)-2,1borazaronaphthalene (5b). Reaction was run at $80^{\circ} \mathrm{C}$ with 3.0 equiv of $\mathrm{B}_{2} \mathrm{Pin}_{2}(381 \mathrm{mg}, 1.5 \mathrm{mmol})$, and the product was obtained as a white solid (160 mg, 76\%, $0.5 \mathrm{mmol} \mathrm{scale);} \mathrm{mp:} 120-124{ }^{\circ} \mathrm{C} ;{ }^{1} \mathrm{H}$ $\operatorname{NMR}\left(\mathrm{CDCl}_{3}, 500.4 \mathrm{MHz}\right): \delta 9.66(\mathrm{~s}, 1 \mathrm{H}), 8.33(\mathrm{~s}, 1 \mathrm{H}), 8.15(\mathrm{~s}, 1 \mathrm{H})$, $7.92(\mathrm{~d}, J=11.7 \mathrm{~Hz}, 1 \mathrm{H}), 6.57(\mathrm{~d}, J=11.9 \mathrm{~Hz}, 1 \mathrm{H}), 1.42(\mathrm{~s}, 12 \mathrm{H})$, $1.37(\mathrm{~s}, 12 \mathrm{H}), 0.90-0.87(\mathrm{~m}, 2 \mathrm{H}), 0.63(\mathrm{dd}, J=6.0,2.2 \mathrm{~Hz}, 2 \mathrm{H})$, $0.40-0.28(\mathrm{~m}, 1 \mathrm{H}) \mathrm{ppm} ;{ }^{13} \mathrm{C}\left\{{ }^{1} \mathrm{H}\right\} \operatorname{NMR}\left(\mathrm{CDCl}_{3}, 125.8 \mathrm{MHz}\right): \delta$ 148.1, 144.8, 142.6, 140.7, 124.1, 83.9, 83.5, 24.9, 24.8, 14.0, 6.3, 6.0 ppm; ${ }^{11} \mathrm{~B}$ NMR $\left(\mathrm{CDCl}_{3}, 128.4 \mathrm{MHz}\right): \delta 38.0,30.2$ (br, 2B) ppm; IR: $\nu$ $=3358,2978,1605,1360,1342,1313,1138,851,774 \mathrm{~cm}^{-1}$; HRMS (ESI) $m / z$ calcd for $\mathrm{C}_{23} \mathrm{H}_{35} \mathrm{~B}_{3} \mathrm{NO}_{4}[\mathrm{M}+\mathrm{H}]^{+} 422.2845$, found 422.2849 .

2-Methyl-6,8-bis(4,4,5,5-tetramethyl-1,3,2-dioxaboryl)-2,1-borazaronaphthalene $(5 \mathrm{C})$. Reaction was run at $80{ }^{\circ} \mathrm{C}$ with 3.0 equiv of $\mathrm{B}_{2} \mathrm{Pin}_{2}(381 \mathrm{mg}, 1.5 \mathrm{mmol})$, and the product was obtained as a white solid (156 mg, 79\%, $0.5 \mathrm{mmol} \mathrm{scale);} \mathrm{mp:} 167-170{ }^{\circ} \mathrm{C} ;{ }^{1} \mathrm{H}$ NMR $\left(\mathrm{CDCl}_{3}, 500.4 \mathrm{MHz}\right): \delta 9.80(\mathrm{~s}, 1 \mathrm{H}), 8.38(\mathrm{~s}, 1 \mathrm{H}), 8.21(\mathrm{~s}, 1 \mathrm{H}), 7.97$ $(\mathrm{d}, J=11.5 \mathrm{~Hz}, 1 \mathrm{H}), 6.80(\mathrm{~d}, J=12.5 \mathrm{~Hz}, 1 \mathrm{H}), 1.44(\mathrm{~s}, 12 \mathrm{H}), 1.39$ (s, $12 \mathrm{H}), 0.81$ (s, 3H) ppm; ${ }^{13} \mathrm{C}\left\{{ }^{1} \mathrm{H}\right\}$ NMR $\left(\mathrm{CDCl}_{3}, 125.8 \mathrm{MHz}\right): \delta$ 148.1, 144.5, 142.6, 140.8, 124.1, 83.9, 83.5, 24.9, 24.8 ppm; ${ }^{11} \mathrm{~B}$ NMR $\left(\mathrm{CDCl}_{3}, 128.4 \mathrm{MHz}\right): \delta 37.6,30.5$ (br, 2B) ppm; IR: $\nu=3354,2981$, $1605,1340,1313,1270,1139,758 \mathrm{~cm}^{-1}$; HRMS (ESI) $\mathrm{m} / z$ calcd for $\mathrm{C}_{21} \mathrm{H}_{33} \mathrm{~B}_{3} \mathrm{NO}_{4}[\mathrm{M}+\mathrm{H}]^{+}$396.2689, found 396.2693.

N-Deuterio-8-(4,4,5,5-tetramethyl-1,3,2-dioxaboryl)-2-(4(trifluoromethyl)phenyl)-2,1-borazaronaphthalene (7). Reaction was run at $40{ }^{\circ} \mathrm{C}$, and the product was obtained as a white solid (81.4 mg, 41\%, $0.5 \mathrm{mmol} \mathrm{scale}$ ); mp: $139-140{ }^{\circ} \mathrm{C} ;{ }^{1} \mathrm{H} \mathrm{NMR}\left(\mathrm{CDCl}_{3}\right.$, $500.4 \mathrm{MHz}): \delta 8.20(\mathrm{~d}, J=11.5 \mathrm{~Hz}, 1 \mathrm{H}), 8.10(\mathrm{~d}, J=7.8 \mathrm{~Hz}, 2 \mathrm{H})$, $8.02(\mathrm{~d}, J=7.1 \mathrm{~Hz}, 1 \mathrm{H}), 7.81(\mathrm{~d}, J=7.7 \mathrm{~Hz}, 1 \mathrm{H}), 7.75(\mathrm{~d}, J=7.9 \mathrm{~Hz}$, $2 \mathrm{H}), 7.30(\mathrm{~d}, J=11.5 \mathrm{~Hz}, 1 \mathrm{H}), 7.24(\mathrm{t}, J=7.4 \mathrm{~Hz}, 1 \mathrm{H}), 1.49(\mathrm{~s}, 12 \mathrm{H})$ ppm; ${ }^{13} \mathrm{C}\left\{{ }^{1} \mathrm{H}\right\}$ NMR $\left(\mathrm{CDCl}_{3}, 125.8 \mathrm{MHz}\right): \delta 146.2,145.5,137.0$, 133.4, 132.9, 131.0 (q, $J=32.2 \mathrm{~Hz}), 125.3,124.7(\mathrm{q}, J=3.7 \mathrm{~Hz}), 124.3$ $(\mathrm{q}, J=272.1 \mathrm{~Hz}), 120.5,84.2,25.0 \mathrm{ppm} ;{ }^{19} \mathrm{~F}\left\{{ }^{1} \mathrm{H}\right\} \mathrm{NMR}\left(\mathrm{CDCl}_{3}\right.$, $470.8 \mathrm{MHz}): \delta-62.7 \mathrm{ppm} ;{ }^{11} \mathrm{~B}$ NMR $\left(\mathrm{CDCl}_{3}, 128.4 \mathrm{MHz}\right): \delta 33.0$ (br, 2B) ppm; IR: $\nu=2983,1603,1555,1374,1323,1305,1163,1109$, $1067,761 \mathrm{~cm}^{-1}$; HRMS (EI) $\mathrm{m} / z$ calcd for $\mathrm{C}_{21} \mathrm{H}_{21} \mathrm{DB}_{2} \mathrm{NO}_{2} \mathrm{~F}_{3}[\mathrm{M}]^{+}$ 400.1852, found 400.1877.

Experimental Procedure for Palladium-Catalyzed CrossCoupling of 8-Borylated 2,1-Borazaronaphthalenes. To a microwave vial with a stir bar were added borylated 2,1borazaronaphthalene (1.0 equiv), solid aryl bromide (1.1 equiv), $\mathrm{K}_{2} \mathrm{CO}_{3}$ (3.0 equiv), and XPhos-Pd-G2 catalyst $(2 \mathrm{~mol} \%)$. The reaction vial was then capped and purged with argon. A solvent mixture of degassed $t$ - $\mathrm{BuOH} / \mathrm{H}_{2} \mathrm{O}(1: 1-2 \mathrm{~mL} / \mathrm{mmol})$ was added followed by any liquid aryl bromides (1.1 equiv). The reaction vessel was then heated at $40{ }^{\circ} \mathrm{C}$ for $18 \mathrm{~h}$. Upon cooling, the solution was washed with $\mathrm{H}_{2} \mathrm{O}$, extracted with $\mathrm{CH}_{2} \mathrm{Cl}_{2}$, further washed with saturated $\mathrm{NH}_{4} \mathrm{Cl}$, dried $\left(\mathrm{Na}_{2} \mathrm{SO}_{4}\right)$, and concentrated in vacuo. If required, the product was further purified by automated column chromatography with silica gel and hexane/EtOAc as eluent to yield 8substituted 2,1-borazaronaphthalenes.

8-(Pyrimidin-5-yl)-2-(4-(trifluoromethyl)phenyl)-2,1-borazaronaphthalene (10a). Obtained as a light brown solid (172 mg, 98\%, $0.5 \mathrm{mmol}$ scale); mp: $97-99{ }^{\circ} \mathrm{C}$; ${ }^{1} \mathrm{H} \mathrm{NMR}\left(\mathrm{CDCl}_{3}, 500.4 \mathrm{MHz}\right): \delta$ 9.39 (s, 1H), 8.99 (s, 2H), 8.27 (d, $J=11.5 \mathrm{~Hz}, 1 \mathrm{H}), 8.19(\mathrm{~s}, 1 \mathrm{H})$, 7.84-7.80 (m, 3H), $7.66(\mathrm{~d}, J=7.9 \mathrm{~Hz}, 2 \mathrm{H}), 7.42(\mathrm{dd}, J=7.2,1.1 \mathrm{~Hz}$ $1 \mathrm{H}), 7.37(\mathrm{t}, J=7.6 \mathrm{~Hz}, 1 \mathrm{H}), 7.33(\mathrm{dd}, J=11.6,1.6 \mathrm{~Hz}, 1 \mathrm{H}) \mathrm{ppm} ;{ }^{13} \mathrm{C}$ $\left\{{ }^{1} \mathrm{H}\right\}$ NMR $\left(\mathrm{CDCl}_{3}, 125.8 \mathrm{MHz}\right): \delta 158.7,158.6,157.5,146.6,137.2$, $132.9,132.2,131.8(\mathrm{q}, J=32.4 \mathrm{~Hz}), 131.2,130.4,126.5,125.1(\mathrm{q}, J=$ $3.6 \mathrm{~Hz}), 124.3(\mathrm{q}, J=272.2 \mathrm{~Hz}), 123.6,121.8 \mathrm{ppm} ;{ }^{19} \mathrm{~F}\left\{{ }^{1} \mathrm{H}\right\} \mathrm{NMR}$ $\left(\mathrm{CDCl}_{3}, 470.8 \mathrm{MHz}\right): \delta-62.9 \mathrm{ppm} ;{ }^{11} \mathrm{~B}$ NMR $\left(\mathrm{CDCl}_{3}, 128.4 \mathrm{MHz}\right)$ : $\delta 33.4$ ppm; IR: $\nu=3409,3285,2980,1568,1323,1162,1117,1065$, $827,757 \mathrm{~cm}^{-1}$; HRMS (ES+) $\mathrm{m} / z$ calcd for $\mathrm{C}_{19} \mathrm{H}_{14} \mathrm{BF}_{3} \mathrm{~N}_{3}[\mathrm{M}+\mathrm{H}]^{+}$ 352.1223 , found 352.1222 .

8-(Pyrimidin-5-yl)-2-(p-tolyl)-2,1-borazaronaphthalene (10b). Obtained as a white solid (100.3 mg, 67\%, $0.5 \mathrm{mmol} \mathrm{scale);} \mathrm{mp:}$ 102-104 ${ }^{\circ} \mathrm{C}$; ${ }^{1} \mathrm{H}$ NMR $\left(\mathrm{CDCl}_{3}, 500.4 \mathrm{MHz}\right): \delta 9.39(\mathrm{~s}, 1 \mathrm{H}), 8.99(\mathrm{~s}$, $2 \mathrm{H}), 8.19(\mathrm{~d}, J=11.6 \mathrm{~Hz}, 1 \mathrm{H}), 8.13(\mathrm{~s}, 1 \mathrm{H}), 7.77(\mathrm{dd}, J=7.8,1.5 \mathrm{~Hz}$, $1 \mathrm{H}), 7.67-7.61(\mathrm{~m}, 2 \mathrm{H}), 7.41-7.28(\mathrm{~m}, 3 \mathrm{H}), 7.24(\mathrm{~d}, J=7.6 \mathrm{~Hz}$, 2H), 2.39 (s, 3H) ppm; ${ }^{13} \mathrm{C}\left\{{ }^{1} \mathrm{H}\right\}$ NMR $\left(\mathrm{CDCl}_{3}, 125.8 \mathrm{MHz}\right): \delta$ $158.5,157.5,145.8,140.3,137.5,132.8,132.5,131.0,130.0,129.3$, 126.4, 123.3, 121.2, $21.7 \mathrm{ppm}^{11} \mathrm{~B}$ NMR $\left(\mathrm{CDCl}_{3}, 128.4 \mathrm{MHz}\right): \delta 32.3$ ppm; IR: $\nu=3403,3031,1597,1549,1436,1401,1191,760,726,721$, $692 \mathrm{~cm}^{-1}$; HRMS (EI) $\mathrm{m} / z$ calcd for $\mathrm{C}_{19} \mathrm{H}_{16} \mathrm{BN}_{3}[\mathrm{M}]^{+}$297.1437, found 297.1453 .

2-Cyclopropyl-8-(pyrimidin-5-yl)-2, 1-borazaronaphthalene (10c). Obtained as a beige solid (60.6 mg, 98\%, $0.25 \mathrm{mmol}$ scale); mp: $135-$ $137{ }^{\circ} \mathrm{C} ;{ }^{1} \mathrm{H}$ NMR $\left(\mathrm{CDCl}_{3}, 500.4 \mathrm{MHz}\right): \delta 9.34(\mathrm{~s}, 1 \mathrm{H}), 8.92(\mathrm{~s}, 2 \mathrm{H})$, $7.95(\mathrm{~d}, J=11.5 \mathrm{~Hz}, 1 \mathrm{H}), 7.66(\mathrm{~d}, J=7.5 \mathrm{~Hz}, 1 \mathrm{H}), 7.59(\mathrm{~s}, 1 \mathrm{H}), 7.29$ $(\mathrm{dd}, J=7.3,1.5 \mathrm{~Hz}, 1 \mathrm{H}), 7.22(\mathrm{t}, J=7.5 \mathrm{~Hz}, 1 \mathrm{H}), 6.49(\mathrm{dd}, J=11.7$, $1.6 \mathrm{~Hz}, 1 \mathrm{H}), 0.83(\mathrm{~m}, 2 \mathrm{H}), 0.55(\mathrm{~m}, 2 \mathrm{H}), 0.11(\mathrm{~m}, 1 \mathrm{H}) \mathrm{ppm} ;{ }^{13} \mathrm{C}\left\{{ }^{1} \mathrm{H}\right\}$ NMR $\left(\mathrm{CDCl}_{3}, 125.8 \mathrm{MHz}\right): \delta 158.3,157.5,144.9,137.4,132.7,130.9$, 129.7, 125.9, 122.6, 120.6, $6.3 \mathrm{ppm} ;{ }^{11} \mathrm{~B} \mathrm{NMR}\left(\mathrm{CDCl}_{3}, 128.4 \mathrm{MHz}\right): \delta$ 36.7 ppm; IR: $\nu=3277,3023,2995,1605,1565,1453,1411,1356$, $827,768 \mathrm{~cm}^{-1}$; HRMS (EI) $\mathrm{m} / z$ calcd for $\mathrm{C}_{15} \mathrm{H}_{14} \mathrm{BN}_{3}[\mathrm{M}]^{+}$247.1281, found 247.1306 .

8-(Pyridin-3-yl)-2-(4-(trifluoromethyl)phenyl)-2,1-borazaronaphthalene (10d). Obtained as a yellow solid $(170 \mathrm{mg}, 97 \%, 0.5 \mathrm{mmol}$ scale); mp: $55-56{ }^{\circ} \mathrm{C} ;{ }^{1} \mathrm{H}$ NMR $\left(\mathrm{CDCl}_{3}, 500.4 \mathrm{MHz}\right): \delta 8.86(\mathrm{~d}, J=$ $1.6 \mathrm{~Hz}, 1 \mathrm{H}), 8.79(\mathrm{dd}, J=4.8,1.5 \mathrm{~Hz}, 1 \mathrm{H}), 8.37(\mathrm{~s}, 1 \mathrm{H}), 8.26(\mathrm{~d}, J=$ $11.5 \mathrm{~Hz}, 1 \mathrm{H}), 7.90(\mathrm{dt}, J=7.7,1.8 \mathrm{~Hz}, 1 \mathrm{H}), 7.81(\mathrm{~d}, J=7.8 \mathrm{~Hz}, 2 \mathrm{H})$, $7.77(\mathrm{~d}, J=7.8 \mathrm{~Hz}, 1 \mathrm{H}), 7.65(\mathrm{~d}, J=7.8 \mathrm{~Hz}, 2 \mathrm{H}), 7.54(\mathrm{dd}, J=7.5,5.0$ $\mathrm{Hz}, 1 \mathrm{H}), 7.44(\mathrm{dd}, J=7.3,1.2 \mathrm{~Hz}, 1 \mathrm{H}), 7.34(\mathrm{t}, J=7.6 \mathrm{~Hz}, 1 \mathrm{H}), 7.30$ (dd, $J=11.6,1.8 \mathrm{~Hz}, 1 \mathrm{H}) \mathrm{ppm} ;{ }^{13} \mathrm{C}\left\{{ }^{1} \mathrm{H}\right\} \mathrm{NMR}\left(\mathrm{CDCl}_{3}, 125.8 \mathrm{MHz}\right)$ : $\delta 150.5,149.8,146.7,137.2,137.1,133.9,132.9,131.5(\mathrm{q}, J=32.3$ $\mathrm{Hz}), 130.3,130.1,127.4,126.3,125.0(\mathrm{q}, J=4.0 \mathrm{~Hz}), 124.2,123.3(\mathrm{q}, J$ $=270.9 \mathrm{~Hz}), 121.6 \mathrm{ppm} ;{ }^{19} \mathrm{~F}\left\{{ }^{1} \mathrm{H}\right\}$ NMR $\left(\mathrm{CDCl}_{3}, 470.8 \mathrm{MHz}\right): \delta$ -62.8 ppm; ${ }^{11} \mathrm{~B}$ NMR $\left(\mathrm{CDCl}_{3}, 128.4 \mathrm{MHz}\right): \delta 33.3 \mathrm{ppm}$; IR: $\nu=$ 3398, 3029, 2970, 2931,1601,1567, 1320,1119, 1104, $1065 \mathrm{~cm}^{-1}$; HRMS (ES+) $m / z$ calcd for $\mathrm{C}_{20} \mathrm{H}_{15} \mathrm{BF}_{3} \mathrm{~N}_{2}[\mathrm{M}+\mathrm{H}]^{+} 351.1280$, found 351.1301.

8-(Quinolin-3-yl)-2-(4-(trifluoromethyl)phenyl)-2, 1-borazaronaphthalene (10e). Obtained as a white solid (197 mg, 98\%, 0.5 mmol scale); mp: $138-140{ }^{\circ} \mathrm{C}$; ${ }^{1} \mathrm{H}$ NMR $\left(\mathrm{CDCl}_{3}, 500.4 \mathrm{MHz}\right): \delta$ $9.15(\mathrm{~d}, J=2.1 \mathrm{~Hz}, 1 \mathrm{H}), 8.49(\mathrm{~s}, 1 \mathrm{H}), 8.38(\mathrm{~d}, J=1.7 \mathrm{~Hz}, 1 \mathrm{H}), 8.29$ $(\mathrm{dd}, J=13.5,10.1 \mathrm{~Hz}, 1 \mathrm{H}), 7.94(\mathrm{~d}, J=8.1 \mathrm{~Hz}, 1 \mathrm{H}), 7.86(\mathrm{t}, J=7.7$ $\mathrm{Hz}, 1 \mathrm{H}), 7.83-7.77(\mathrm{~m}, 3 \mathrm{H}), 7.69(\mathrm{t}, J=7.4 \mathrm{~Hz}, 1 \mathrm{H}), 7.61(\mathrm{~d}, J=7.8$ $\mathrm{Hz}, 1 \mathrm{H}), 7.54(\mathrm{dd}, J=7.3,1.1 \mathrm{~Hz}, 1 \mathrm{H}), 7.39(\mathrm{t}, J=7.6 \mathrm{~Hz}, 1 \mathrm{H}), 7.33$ (dd, $J=11.5,1.6 \mathrm{~Hz}, 1 \mathrm{H}) \mathrm{ppm} ;{ }^{13} \mathrm{C}\left\{{ }^{1} \mathrm{H}\right\} \mathrm{NMR}\left(\mathrm{CDCl}_{3}, 125.8 \mathrm{MHz}\right)$ : $\delta 151.3,147.9,146.7,137.5,136.4,132.9,131.5(\mathrm{q}, J=32.2 \mathrm{~Hz})$, $131.1,130.5,130.4,130.4,129.7,128.1,128.1,127.8,127.5,126.4$, $125.0(\mathrm{q}, J=3.6 \mathrm{~Hz}), 124.4(\mathrm{q}, J=272.0 \mathrm{~Hz}), 121.7 \mathrm{ppm} ;{ }^{19} \mathrm{~F}\left\{{ }^{1} \mathrm{H}\right\}$ NMR $\left(\mathrm{CDCl}_{3}, 470.8 \mathrm{MHz}\right): \delta-62.9 \mathrm{ppm} ;{ }^{11} \mathrm{~B}$ NMR $\left(\mathrm{CDCl}_{3}, 128.4\right.$ $\mathrm{MHz}): \delta 31.6$ ppm; IR: $\nu=3402,3012,2969,2935,1561,1319,1160$, $1110,1065,821,751 \mathrm{~cm}^{-1}$; HRMS (ES+) $\mathrm{m} / z$ calcd for $\mathrm{C}_{24} \mathrm{H}_{17} \mathrm{BF}_{3} \mathrm{~N}_{2}$ $[\mathrm{M}+\mathrm{H}]^{+}$401.1437, found 401.1426.

8-(Thiophen-3-yl)-2-(4-(trifluoromethyl)phenyl)-2,1-borazaronaphthalene (10f). Obtained as a white solid $(156.3 \mathrm{mg}, 88 \%, 0.5$ mmol scale); mp: $95-96{ }^{\circ} \mathrm{C}$; ${ }^{1} \mathrm{H}$ NMR $\left(\mathrm{CDCl}_{3}, 500.4 \mathrm{MHz}\right): \delta 8.79$ 
(s, 1H), 8.25 (d, $J=11.5 \mathrm{~Hz}, 1 \mathrm{H}), 7.87(\mathrm{~d}, J=7.8 \mathrm{~Hz}, 2 \mathrm{H}), 7.73-7.66$ $(\mathrm{m}, 3 \mathrm{H}), 7.63(\mathrm{dd}, J=4.9,3.0 \mathrm{~Hz}, 1 \mathrm{H}), 7.53-7.49(\mathrm{~m}, 2 \mathrm{H}), 7.36(\mathrm{dd}$, $J=4.9,1.2 \mathrm{~Hz}, 1 \mathrm{H}), 7.32-7.27(\mathrm{~m}, 2 \mathrm{H}) \mathrm{ppm} ;{ }^{13} \mathrm{C}\left\{{ }^{1} \mathrm{H}\right\} \mathrm{NMR}$ $\left(\mathrm{CDCl}_{3}, 125.8 \mathrm{MHz}\right): \delta 146.4,138.2,137.3,132.7(2 \times \mathrm{C}), 131.2(\mathrm{q}, J$ $=32.2 \mathrm{~Hz}), 129.4,129.2,128.3,127.5,125.9,124.8(\mathrm{q}, J=3.8 \mathrm{~Hz})$, $124.2(\mathrm{q}, J=272.0 \mathrm{~Hz}), 123.6,121.1 \mathrm{ppm} ;{ }^{19} \mathrm{~F}\left\{{ }^{1} \mathrm{H}\right\} \operatorname{NMR}\left(\mathrm{CDCl}_{3}\right.$, $470.8 \mathrm{MHz}): \delta-62.7 \mathrm{ppm} ;{ }^{11} \mathrm{~B} \mathrm{NMR}\left(\mathrm{CDCl}_{3}, 128.4 \mathrm{MHz}\right): \delta 33.0$ ppm; IR: $\nu=3397,3096,3047,3016,1600,1566,1324,1102,1066$, $752 \mathrm{~cm}^{-1}$; HRMS (EI) $\mathrm{m} / z$ calcd for $\mathrm{C}_{19} \mathrm{H}_{13} \mathrm{BF}_{3} \mathrm{NS}[\mathrm{M}]^{+}$355.0814, found 355.0829 .

8-(Thiophen-3-yl)-2-(3-methoxyphenyl)-2,1-borazaronaphthalene $(10 \mathrm{~g})$. Obtained as a white solid $(150 \mathrm{mg}, 95 \%, 0.5 \mathrm{mmol} \mathrm{scale})$; mp: $85-86{ }^{\circ} \mathrm{C} ;{ }^{1} \mathrm{H}$ NMR $\left(\mathrm{CDCl}_{3}, 500.4 \mathrm{MHz}\right): \delta 8.76(\mathrm{~s}, 1 \mathrm{H}), 8.20$ $(\mathrm{d}, J=11.5 \mathrm{~Hz}, 1 \mathrm{H}), 7.67(\mathrm{~d}, J=7.8 \mathrm{~Hz}, 1 \mathrm{H}), 7.61(\mathrm{dd}, J=4.7,3.0$ $\mathrm{Hz}, 1 \mathrm{H}), 7.51(\mathrm{~d}, J=2.1 \mathrm{~Hz}, 1 \mathrm{H}), 7.48(\mathrm{dd}, J=7.5,1.1 \mathrm{~Hz}, 1 \mathrm{H})$, $7.40-7.34(\mathrm{~m}, 3 \mathrm{H}), 7.33(\mathrm{~d}, J=2.5 \mathrm{~Hz}, 1 \mathrm{H}), 7.29(\mathrm{dd}, J=11.5,1.7$ $\mathrm{Hz}, 1 \mathrm{H}), 7.24(\mathrm{t}, J=7.7 \mathrm{~Hz}, 1 \mathrm{H}), 6.98(\mathrm{dt}, J=7.3,2.3 \mathrm{~Hz}, 1 \mathrm{H}), 3.86$ (s, 3H) ppm; ${ }^{13} \mathrm{C}\left\{{ }^{1} \mathrm{H}\right\}$ NMR $\left(\mathrm{CDCl}_{3}, 125.8 \mathrm{MHz}\right): \delta 159.7,146.1$, 138.6, 137.8, 129.6, 129.4, 129.3, 128.7, 127.5, 126.1, 126.0, 125.1, $123.8,120.9,118.2,115.2,55.3 \mathrm{ppm} ;{ }^{11} \mathrm{~B} \mathrm{NMR}\left(\mathrm{CDCl}_{3}, 128.4 \mathrm{MHz}\right)$ : $\delta 34.7$ ppm; IR: $\nu=3384,2920,1597,1565,1436,1264,1255,755$, $720 \mathrm{~cm}^{-1}$; HRMS $(\mathrm{ES}+) \mathrm{m} / z$ calcd for $\mathrm{C}_{19} \mathrm{H}_{17} \mathrm{BNOS}[\mathrm{M}+\mathrm{H}]^{+}$ 318.1124 , found 318.1106 .

2-(4-(Trifluoromethyl)phenyl)-8-(1,3,7-trimethyl-2,6-dioxo2,3,6,7-tetrahydro-1H-purin-8-yl)-2,1-borazaronaphthalene (10h). Obtained as a white solid (233 mg, 99\%, $0.5 \mathrm{mmol} \mathrm{scale})$; $\mathrm{mp}$ : $>260{ }^{\circ} \mathrm{C} ;{ }^{1} \mathrm{H}$ NMR $\left(\mathrm{CDCl}_{3}, 500.4 \mathrm{MHz}\right): \delta 11.04(\mathrm{~s}, 1 \mathrm{H}), 8.26(\mathrm{~d}, J=$ $11.6 \mathrm{~Hz}, 1 \mathrm{H}), 8.04(\mathrm{~d}, J=7.7 \mathrm{~Hz}, 2 \mathrm{H}), 7.87(\mathrm{~d}, J=7.7 \mathrm{~Hz}, 1 \mathrm{H}), 7.70$ $(\mathrm{d}, J=7.9 \mathrm{~Hz}, 2 \mathrm{H}), 7.67(\mathrm{dd}, J=7.7,1.2 \mathrm{~Hz}, 1 \mathrm{H}), 7.44-7.32(\mathrm{~m}, 2 \mathrm{H})$, $4.15(\mathrm{~s}, 3 \mathrm{H}), 3.76(\mathrm{~s}, 3 \mathrm{H}), 3.49(\mathrm{~s}, 3 \mathrm{H}) \mathrm{ppm} ;{ }^{13} \mathrm{C}\left\{{ }^{1} \mathrm{H}\right\} \mathrm{NMR}\left(\mathrm{CDCl}_{3}\right.$, $125.8 \mathrm{MHz}): \delta 155.6,151.8,149.6,148.0,146.3,139.3,133.2,132.7$, $131.8(\mathrm{q}, J=32.3 \mathrm{~Hz}), 129.5,127.2,125.1(\mathrm{q}, J=3.4 \mathrm{~Hz}), 124.4(\mathrm{q}, J$ $=272.1 \mathrm{~Hz}), 120.4,115.0,108.8,35.1,30.1,28.4 \mathrm{ppm} ;{ }^{19} \mathrm{~F}\left\{{ }^{1} \mathrm{H}\right\}$ NMR $\left(\mathrm{CDCl}_{3}, 470.8 \mathrm{MHz}\right): \delta-62.8 \mathrm{ppm} ;{ }^{11} \mathrm{~B}$ NMR $\left(\mathrm{CDCl}_{3}, 128.4 \mathrm{MHz}\right)$ : $\delta 33.0 \mathrm{ppm}$; IR: $\nu=3312,2950,1698,1658,1539,1323,1164,1108$, $1067 \mathrm{~cm}^{-1}$; HRMS (ES+) $\mathrm{m} / z$ calcd for $\mathrm{C}_{23} \mathrm{H}_{20} \mathrm{BF}_{3} \mathrm{~N}_{5} \mathrm{O}_{2}[\mathrm{M}+\mathrm{H}]^{+}$ 466.1662 , found 466.1668 .

2-(4-Methoxyphenyl)-8-(1,3,7-trimethyl-2,6-dioxo-2,3,6,7-tetrahydro-1H-purin-8-yl)-2,1-borazaronaphthalene (10i). Recrystallized from toluene and obtained as a white solid $(212 \mathrm{mg}, 99 \%, 0.5 \mathrm{mmol}$ scale); mp: $>260{ }^{\circ} \mathrm{C} ;{ }^{1} \mathrm{H} \mathrm{NMR}\left(\mathrm{CDCl}_{3}, 500.4 \mathrm{MHz}\right): \delta 10.76(\mathrm{~s}, 1 \mathrm{H})$, $8.14(\mathrm{~d}, J=11.6 \mathrm{~Hz}, 1 \mathrm{H}), 7.89(\mathrm{~d}, J=8.5 \mathrm{~Hz}, 2 \mathrm{H}), 7.79(\mathrm{~d}, J=7.7 \mathrm{~Hz}$, $1 \mathrm{H}), 7.61(\mathrm{dd}, J=7.2,1.0 \mathrm{~Hz}, 1 \mathrm{H}), 7.36(\mathrm{dd}, J=11.5,1.5 \mathrm{~Hz}, 1 \mathrm{H})$, $7.29(\mathrm{t}, J=7.7 \mathrm{~Hz}, 1 \mathrm{H}), 7.00(\mathrm{~d}, J=8.5 \mathrm{~Hz}, 2 \mathrm{H}), 4.13(\mathrm{~s}, 3 \mathrm{H}), 3.87(\mathrm{~s}$, $3 \mathrm{H}), 3.77(\mathrm{~s}, 3 \mathrm{H}), 3.49(\mathrm{~s}, 3 \mathrm{H}) .{ }^{13} \mathrm{C}\left\{{ }^{1} \mathrm{H}\right\} \mathrm{NMR}\left(\mathrm{CDCl}_{3}, 125.8 \mathrm{MHz}\right)$ : $\delta 161.3,155.3,151.6,149.7,147.7,145.0,139.5,134.4,132.2,128.9$, 126.7, 119.4, 114.3, 113.8, 108.4, 55.1, 34.7, 29.7, 28.3 ppm; ${ }^{11} \mathrm{~B}$ NMR $\left(\mathrm{CDCl}_{3}, 128.4 \mathrm{MHz}\right): \delta 31.6 \mathrm{ppm}$; IR: $\nu=3305,2939,1703,1662$, $1598,1442,1243,1179,1026 \mathrm{~cm}^{-1}$; HRMS (EI) $\mathrm{m} / z$ calcd for $\mathrm{C}_{23} \mathrm{H}_{23} \mathrm{BN}_{5} \mathrm{O}_{3}[\mathrm{M}+\mathrm{H}]^{+}$428.1894, found 428.1891.

6-Cyano-2-(4-(trifluoromethyl)phenyl)-8-(1,3,7-trimethyl-2,6dioxo-2,3,6,7-tetrahydro-1H-purin-8-yl)-2,1-borazaronaphthalene (10j). Obtained as a light yellow solid $(79.7 \mathrm{mg}, 65 \%, 0.25 \mathrm{mmol}$ scale); mp: $>260{ }^{\circ} \mathrm{C} ;{ }^{1} \mathrm{H}$ NMR $\left(\mathrm{CDCl}_{3}, 500.4 \mathrm{MHz}\right): \delta 11.35$ (s, $\left.1 \mathrm{H}\right)$, $8.23(\mathrm{~d}, J=11.6 \mathrm{~Hz}, 1 \mathrm{H}), 8.17(\mathrm{~s}, 1 \mathrm{H}), 8.04(\mathrm{~d}, J=7.5 \mathrm{~Hz}, 2 \mathrm{H}), 7.88$ $(\mathrm{d}, J=1.5 \mathrm{~Hz}, 1 \mathrm{H}), 7.73(\mathrm{~d}, J=7.8 \mathrm{~Hz}, 2 \mathrm{H}), 7.53(\mathrm{~d}, J=11.5 \mathrm{~Hz}$, $1 \mathrm{H}), 4.20(\mathrm{~s}, 3 \mathrm{H}), 3.75(\mathrm{~s}, 3 \mathrm{H}), 3.49(\mathrm{~s}, 3 \mathrm{H}) \mathrm{ppm} ;{ }^{13} \mathrm{C}\left\{{ }^{1} \mathrm{H}\right\} \mathrm{NMR}$ $\left(\mathrm{CDCl}_{3}, 125.8 \mathrm{MHz}\right): \delta 155.5,151.6,147.8,147.1,145.4,141.8,136.4$, 133.4, $132.5(\mathrm{q}, J=32.4 \mathrm{~Hz}), 131.1,127.2,125.2(\mathrm{q}, J=3.8 \mathrm{~Hz})$, $124.18(\mathrm{q}, J=118.4 \mathrm{~Hz}), 116.3,109.1,104.1,35.1,30.0,28.4 \mathrm{ppm} ;{ }^{11} \mathrm{~B}$ NMR $\left(\mathrm{CDCl}_{3}, 128.4 \mathrm{MHz}\right): \delta 34.0 \mathrm{ppm} ;{ }^{19} \mathrm{~F}\left\{{ }^{1} \mathrm{H}\right\}$ NMR $\left(\mathrm{CDCl}_{3}\right.$, $470.8 \mathrm{MHz}): \delta-62.9 \mathrm{ppm}$; IR: $\nu=3308,2962,2230,1712,1674$, 1327, 1166, 1101, 1070, $779 \mathrm{~cm}^{-1}$; HRMS (ES+) $\mathrm{m} / z$ calcd for $\mathrm{C}_{24} \mathrm{H}_{19} \mathrm{BN}_{6} \mathrm{O}_{2} \mathrm{~F}_{3}[\mathrm{M}+\mathrm{H}]^{+}$491.1615, found 491.1636.

6-(Trifluoromethoxy)-2-(4-(trifluoromethyl)phenyl)-8-(1,3,7-trimethyl-2,6-dioxo-2,3,6,7-tetrahydro-1H-purin-8-yl)-2,1-borazaronaphthalene (10k). Obtained as a white solid (81 mg, 58\%, 0.25 mmol scale); mp: $209-210{ }^{\circ} \mathrm{C} ;{ }^{1} \mathrm{H}$ NMR $\left(\mathrm{CDCl}_{3}, 500.4 \mathrm{MHz}\right): \delta$ $11.13(\mathrm{~s}, 1 \mathrm{H}), 8.21(\mathrm{~d}, J=11.6 \mathrm{~Hz}, 1 \mathrm{H}), 8.03(\mathrm{~d}, J=7.8 \mathrm{~Hz}, 2 \mathrm{H})$, $7.74-7.67(\mathrm{~m}, 3 \mathrm{H}), 7.55(\mathrm{~d}, J=2.3 \mathrm{~Hz}, 1 \mathrm{H}), 7.47(\mathrm{dd}, J=11.6,1.6$
$\mathrm{Hz}, 1 \mathrm{H}), 4.17$ (s, 3H), $3.75(\mathrm{~s}, 3 \mathrm{H}), 3.48(\mathrm{~s}, 3 \mathrm{H}) \mathrm{ppm} ;{ }^{13} \mathrm{C}\left\{{ }^{1} \mathrm{H}\right\} \mathrm{NMR}$ $\left(\mathrm{CDCl}_{3}, 125.8 \mathrm{MHz}\right): \delta$ 155.5, 151.6, 147.9, 147.8, 145.5, 141.8, 137.9, $133.2,132.1(\mathrm{q}, J=32.5 \mathrm{~Hz}), 127.7,125.1(\mathrm{q}, J=3.7 \mathrm{~Hz}), 124.2,124.0$ $(\mathrm{q}, J=274.8 \mathrm{~Hz}), 122.5,120.8(\mathrm{q}, J=257.6 \mathrm{~Hz}), 116.0,109.0,34.9$, 30.0, $28.3 \mathrm{ppm} ;{ }^{19} \mathrm{~F}\left\{{ }^{1} \mathrm{H}\right\}$ NMR $\left(\mathrm{CDCl}_{3}, 470.8 \mathrm{MHz}\right): \delta-58.3,-62.9$ ppm; ${ }^{11} \mathrm{~B}$ NMR $\left(\mathrm{CDCl}_{3}, 128.4 \mathrm{MHz}\right): \delta 32.8 \mathrm{ppm}$; IR: $\nu=3302$, 3047, 2951, 1702, 1663, 1326, 1267, 1257, 1166, $1106 \mathrm{~cm}^{-1}$; HRMS (ES+) $m / z$ calcd for $\mathrm{C}_{24} \mathrm{H}_{19} \mathrm{BN}_{5} \mathrm{O}_{3} \mathrm{~F}_{6}[\mathrm{M}+\mathrm{H}]^{+} 550.1485$, found 550.1480 .

8-(4-(2,1-Borazaronaphthalen-2-yl)phenyl)-2-(4(trifluoromethyl)phenyl)-2,1-borazaronaphthalene (10l). Obtained as an off white solid $(202 \mathrm{mg}, 85 \%, 0.5 \mathrm{mmol} \mathrm{scale})$; mp: $138-140{ }^{\circ} \mathrm{C}$; ${ }^{1} \mathrm{H}$ NMR $\left(\mathrm{CDCl}_{3}, 500.4 \mathrm{MHz}\right): \delta 8.76(\mathrm{~s}, 1 \mathrm{H}), 8.21-8.29(\mathrm{~m}, 3 \mathrm{H})$, $8.16(\mathrm{~d}, J=7.6 \mathrm{~Hz}, 2 \mathrm{H}), 7.84(\mathrm{~d}, J=7.8 \mathrm{~Hz}, 2 \mathrm{H}), 7.73(\mathrm{t}, J=9.9 \mathrm{~Hz}$, $2 \mathrm{H}), 7.70(\mathrm{~d}, J=7.7 \mathrm{~Hz}, 2 \mathrm{H}), 7.66(\mathrm{~d}, J=7.8 \mathrm{~Hz}, 2 \mathrm{H}), 7.52(\mathrm{~d}, J=7.0$ $\mathrm{Hz}, 1 \mathrm{H}), 7.49(\mathrm{t}, J=7.2 \mathrm{~Hz}, 1 \mathrm{H}), 7.41(\mathrm{~d}, J=8.8 \mathrm{~Hz}, 1 \mathrm{H}), 7.39-7.29$ $(\mathrm{m}, 3 \mathrm{H}), 7.26(\mathrm{t}, J=7.8 \mathrm{~Hz}, 1 \mathrm{H}) \mathrm{ppm} ;{ }^{13} \mathrm{C}\left\{{ }^{1} \mathrm{H}\right\} \mathrm{NMR}\left(\mathrm{CDCl}_{3}, 125.8\right.$ MHz): $\delta 146.7,146.1,140.3,139.3,137.2,133.9,133.0,131.4$ (q, $J=$ $30.9 \mathrm{~Hz}), 131.2,129.8,129.8,129.5,129.4,128.8,128.1,126.2,126.0$, 125.0, $125.0(\mathrm{q}, J=4.6 \mathrm{~Hz}), 124.5(\mathrm{q}, J=273.0 \mathrm{~Hz}), 121.5,121.4$, $118.5 \mathrm{ppm} ;{ }^{19} \mathrm{~F}\left\{{ }^{1} \mathrm{H}\right\}$ NMR $\left(\mathrm{CDCl}_{3}, 470.8 \mathrm{MHz}\right): \delta-62.8 \mathrm{ppm} ;{ }^{11} \mathrm{~B}$ NMR $\left(\mathrm{CDCl}_{3}, 128.4 \mathrm{MHz}\right): \delta 33.8$ ppm; IR: $\nu=3385,3397,2954$, $2931,1598,1562,1437,1322,1167,1117,1066,808,757 \mathrm{~cm}^{-1}$; HRMS (EI) $m / z$ calcd for $\mathrm{C}_{29} \mathrm{H}_{21} \mathrm{~B}_{2} \mathrm{~F}_{3} \mathrm{~N}_{2}[\mathrm{M}]^{+} 476.1843$, found 476.1857 .

8-(2-Methyl-2,1-borazaronaphthalen-3-yl)-2-(4-(trifluoromethyl)phenyl)-2,1-borazaronaphthalene (10m). Obtained as a white solid (70 mg, 68\%, $0.25 \mathrm{mmol} \mathrm{scale})$; mp: $159-161{ }^{\circ} \mathrm{C} ;{ }^{1} \mathrm{H} \mathrm{NMR}\left(\mathrm{CDCl}_{3}\right.$, $500.4 \mathrm{MHz}): \delta 8.42(\mathrm{~s}, 1 \mathrm{H}), 8.28(\mathrm{~d}, J=11.5 \mathrm{~Hz}, 1 \mathrm{H}), 8.04(\mathrm{~s}, 1 \mathrm{H})$, $8.01(\mathrm{~s}, 1 \mathrm{H}), 7.81(\mathrm{~d}, J=7.7 \mathrm{~Hz}, 2 \mathrm{H}), 7.68(\mathrm{t}, J=6.1 \mathrm{~Hz}, 2 \mathrm{H}), 7.61(\mathrm{~d}$, $J=7.7 \mathrm{~Hz}, 2 \mathrm{H}), 7.52(\mathrm{t}, J=7.6 \mathrm{~Hz}, 1 \mathrm{H}), 7.39-7.33(\mathrm{~m}, 2 \mathrm{H}), 7.33-$ $7.22(\mathrm{~m}, 3 \mathrm{H}), 0.64(\mathrm{~s}, 3 \mathrm{H}) \mathrm{ppm} ;{ }^{13} \mathrm{C}\left\{{ }^{1} \mathrm{H}\right\} \mathrm{NMR}\left(\mathrm{CDCl}_{3}, 125.8\right.$ $\mathrm{MHz}): \delta 146.8,144.6,140.3,137.2,134.0,132.9,131.2(\mathrm{q}, J=32.3$ $\mathrm{Hz}), 129.8,129.1,128.9,128.2,125.8,124.9$ (q, $J=3.2 \mathrm{~Hz}), 124.5$ (q, $J$ $=272.0 \mathrm{~Hz}), 121.5,121.2,117.9 \mathrm{ppm} ;{ }^{19} \mathrm{~F}\left\{{ }^{1} \mathrm{H}\right\}$ NMR $\left(\mathrm{CDCl}_{3}, 470.8\right.$ $\mathrm{MHz}): \delta-62.8 \mathrm{ppm} ;{ }^{11} \mathrm{~B}$ NMR $\left(\mathrm{CDCl}_{3}, 128.4 \mathrm{MHz}\right): \delta 35.57,30.9$ ppm; IR: $\nu=3377,1559,1320,1101,1063,759,752,717 \mathrm{~cm}^{-1}$; HRMS (EI) $m / z$ calcd for $\mathrm{C}_{24} \mathrm{H}_{19} \mathrm{~B}_{2} \mathrm{~N}_{2} \mathrm{~F}_{3}[\mathrm{M}]^{+} 414.1686$, found 414.1686

8-(p-Tolyl)-2-(4-(trifluoromethyl)phenyl)-2,1-borazaronaphthalene (10n). Obtained as a white solid $(162.1 \mathrm{mg}, 89 \%, 0.5 \mathrm{mmol}$ scale); mp: $92-93{ }^{\circ} \mathrm{C} ;{ }^{1} \mathrm{H}$ NMR $\left(\mathrm{CDCl}_{3}, 500.4 \mathrm{MHz}\right): \delta 8.70(\mathrm{~s}, 1 \mathrm{H})$, $8.26(\mathrm{~d}, J=11.5 \mathrm{~Hz}, 1 \mathrm{H}), 7.84(\mathrm{~d}, J=7.8 \mathrm{~Hz}, 2 \mathrm{H}), 7.71(\mathrm{~d}, J=7.8 \mathrm{~Hz}$, $1 \mathrm{H}), 7.67(\mathrm{~d}, J=7.9 \mathrm{~Hz}, 2 \mathrm{H}), 7.49-7.43(\mathrm{~m}, 3 \mathrm{H}), 7.41(\mathrm{~d}, J=7.9 \mathrm{~Hz}$, $2 \mathrm{H}), 7.30(\mathrm{t}, J=7.6 \mathrm{~Hz}, 2 \mathrm{H}), 2.51(\mathrm{~s}, 3 \mathrm{H}) \mathrm{ppm} ;{ }^{13} \mathrm{C}\left\{{ }^{1} \mathrm{H}\right\} \mathrm{NMR}$ $\left(\mathrm{CDCl}_{3}, 125.8 \mathrm{MHz}\right): \delta 146.4,138.0,137.1,134.8,132.7,131.2(\mathrm{q}, J=$ $32.3 \mathrm{~Hz}$ ), 131.1, 130.1, 129.5, 129.2, 128.9, 125.9, 124.7 (q, $J=3.8$ $\mathrm{Hz}), 123.2(\mathrm{q}, J=271.9 \mathrm{~Hz}), 121.1,21.2 \mathrm{ppm} ;{ }^{19} \mathrm{~F}\left\{{ }^{1} \mathrm{H}\right\} \mathrm{NMR}$ $\left(\mathrm{CDCl}_{3}, 470.8 \mathrm{MHz}\right): \delta-62.8 \mathrm{ppm} ;{ }^{11} \mathrm{~B} \mathrm{NMR}\left(\mathrm{CDCl}_{3}, 128.4 \mathrm{MHz}\right)$ : $\delta 33.1$ ppm; IR: $\nu=3403,3031,2923,2856,1600,1567,1322,1104$, 1065, $753 \mathrm{~cm}^{-1}$; HRMS (EI) $\mathrm{m} / z$ calcd for $\mathrm{C}_{22} \mathrm{H}_{17} \mathrm{BF}_{3} \mathrm{~N}[\mathrm{M}]^{+}$ 363.1406, found 363.1399 .

2,8-Bis(4-(trifluoromethyl)phenyl)-2,1-borazaronaphthalene (10o). Obtained as a white solid (204 mg, 98\%, $0.5 \mathrm{mmol}$ scale); mp: 105-106 ${ }^{\circ} \mathrm{C} ;{ }^{1} \mathrm{H}$ NMR $\left(\mathrm{CDCl}_{3}, 500.4 \mathrm{MHz}\right): \delta 8.47(\mathrm{~s}, 1 \mathrm{H}), 8.27(\mathrm{~d}$, $J=11.5 \mathrm{~Hz}, 1 \mathrm{H}), 7.87(\mathrm{~d}, J=8.1 \mathrm{~Hz}, 2 \mathrm{H}), 7.81(\mathrm{~d}, J=7.8 \mathrm{~Hz}, 2 \mathrm{H})$, $7.76(\mathrm{~d}, J=7.7 \mathrm{~Hz}, 1 \mathrm{H}), 7.71(\mathrm{~d}, J=8.0 \mathrm{~Hz}, 2 \mathrm{H}), 7.67(\mathrm{~d}, J=7.9 \mathrm{~Hz}$, $2 \mathrm{H}), 7.44(\mathrm{dd}, J=7.3,1.3 \mathrm{~Hz}, 1 \mathrm{H}), 7.38-7.27(\mathrm{~m}, 2 \mathrm{H}) \mathrm{ppm} ;{ }^{13} \mathrm{C}$ $\left\{{ }^{1} \mathrm{H}\right\}$ NMR $\left(\mathrm{CDCl}_{3}, 125.8 \mathrm{MHz}\right): \delta{ }^{13} \mathrm{C}$ NMR $\left(126 \mathrm{MHz}, \mathrm{CDCl}_{3}\right) \delta$ $146.7,141.9,136.9,132.9,131.4(\mathrm{q}, J=32.3 \mathrm{~Hz}), 130.6(\mathrm{q}, J=32.5$ $\mathrm{Hz}), 130.2,130.1,129.9,129.7,126.6(\mathrm{q}, J=3.7 \mathrm{~Hz}), 126.3,125.0(\mathrm{q}, J$ $=3.8 \mathrm{~Hz}), 124.4(\mathrm{q}, J=271.9 \mathrm{~Hz}), 124.2(\mathrm{q}, J=272.0 \mathrm{~Hz}), 121.5$ ppm; ${ }^{19} \mathrm{~F}\left\{{ }^{1} \mathrm{H}\right\}$ NMR $\left(\mathrm{CDCl}_{3}, 470.8 \mathrm{MHz}\right): \delta-62.5,-62.8 \mathrm{ppm} ;{ }^{11} \mathrm{~B}$ NMR $\left(\mathrm{CDCl}_{3}, 128.4 \mathrm{MHz}\right): \delta 31.5$ ppm; IR: $\nu=3405,3035,2939$, $1566,1323,1098,1067,1016,818,755 \mathrm{~cm}^{-1}$; HRMS (EI) $\mathrm{m} / z$ calcd for $\mathrm{C}_{22} \mathrm{H}_{14} \mathrm{BNF}_{6}[\mathrm{M}]^{+}$417.1123, found 417.1129.

8-(4-(Dimethylamino)phenyl)-2-(4-(trifluoromethyl)phenyl)-2,1borazaronaphthalene (10p). Obtained as a white solid (180.4 mg, 92\%, $0.5 \mathrm{mmol}$ scale); mp: $114-115{ }^{\circ} \mathrm{C}$; ${ }^{1} \mathrm{H}$ NMR $\left(\mathrm{CDCl}_{3}, 500.4\right.$ $\mathrm{MHz}): \delta 8.82(\mathrm{~s}, 1 \mathrm{H}), 8.25(\mathrm{~d}, J=11.5 \mathrm{~Hz}, 1 \mathrm{H}), 7.86(\mathrm{~d}, J=7.8 \mathrm{~Hz}$, 
$2 \mathrm{H}), 7.68-7.63(\mathrm{~m}, 3 \mathrm{H}), 7.47-7.41(\mathrm{~m}, 3 \mathrm{H}), 7.30-7.22(\mathrm{~m}, 2 \mathrm{H})$, $6.93(\mathrm{~d}, J=8.5 \mathrm{~Hz}, 2 \mathrm{H}), 3.08(\mathrm{~s}, 6 \mathrm{H}) \mathrm{ppm} ;{ }^{13} \mathrm{C}\left\{{ }^{1} \mathrm{H}\right\} \mathrm{NMR}\left(\mathrm{CDCl}_{3}\right.$, $125.8 \mathrm{MHz}): \delta 150.1,146.5,137.3,132.8,132.6,131.4,131.0(\mathrm{q}, J=$ $32.2 \mathrm{~Hz}), 130.1,129.5,128.3,126.4(\mathrm{q}, J=272.1 \mathrm{~Hz}), 125.8,124.7(\mathrm{q}$, $J=3.9 \mathrm{~Hz}), 121.1,112.9,40.4 \mathrm{ppm} ;{ }^{19} \mathrm{~F}\left\{{ }^{1} \mathrm{H}\right\}$ NMR $\left(\mathrm{CDCl}_{3}, 470.8\right.$ $\mathrm{MHz}): \delta 62.8 \mathrm{ppm} ;{ }^{11} \mathrm{~B}$ NMR $\left(\mathrm{CDCl}_{3}, 128.4 \mathrm{MHz}\right): \delta 32.9$ ppm; IR: $\nu=3386,3045,2893,2804,1601,1565,1522,1321,1115,1103,1064$ $\mathrm{cm}^{-1}$; HRMS (ES+) $\mathrm{m} / z$ calcd for $\mathrm{C}_{23} \mathrm{H}_{21} \mathrm{BF}_{3} \mathrm{~N}_{2}[\mathrm{M}]^{+}$393.1750, found 393.1763 .

8-(4-(Dimethylamino)phenyl)-2-(4-methoxyphenyl)-2,1-borazaronaphthalene (10q). Obtained as a light gray solid (161 mg, 91\%, 0.5 mmol scale); mp: $140-142{ }^{\circ} \mathrm{C} ;{ }^{1} \mathrm{H}$ NMR $\left(\mathrm{CDCl}_{3}, 500.4 \mathrm{MHz}\right): \delta$ $8.71(\mathrm{~s}, 1 \mathrm{H}), 8.18(\mathrm{~d}, J=11.6 \mathrm{~Hz}, 1 \mathrm{H}), 7.75(\mathrm{~d}, J=8.5 \mathrm{~Hz}, 2 \mathrm{H}), 7.62$ $(\mathrm{d}, J=7.6 \mathrm{~Hz}, 1 \mathrm{H}), 7.47(\mathrm{~d}, J=8.7 \mathrm{~Hz}, 2 \mathrm{H}), 7.40(\mathrm{dd}, J=7.3,1.4 \mathrm{~Hz}$, $1 \mathrm{H}), 7.29(\mathrm{~d}, J=11.8 \mathrm{~Hz}, 1 \mathrm{H}), 7.23(\mathrm{t}, J=7.5 \mathrm{~Hz}, 1 \mathrm{H}), 6.99(\mathrm{~d}, J=$ $8.5 \mathrm{~Hz}, 2 \mathrm{H}), 6.95(\mathrm{~d}, J=8.4 \mathrm{~Hz}, 1 \mathrm{H}), 3.86(\mathrm{~s}, 3 \mathrm{H}), 3.09(\mathrm{~s}, 6 \mathrm{H}) \mathrm{ppm}$; ${ }^{13} \mathrm{C}\left\{{ }^{1} \mathrm{H}\right\}$ NMR $\left(\mathrm{CDCl}_{3}, 125.8 \mathrm{MHz}\right): \delta 161.1,150.4,145.8,137.9$, 134.4, 131.3, 130.4, 129.5, 128.4, 125.8 (d, $J=5.2 \mathrm{~Hz}), 120.7,114.0$, 113.2, 77.0, 55.3, $40.7 \mathrm{ppm}$; ${ }^{11} \mathrm{~B}$ NMR $\left(\mathrm{CDCl}_{3}, 128.4 \mathrm{MHz}\right): \delta 33.8$ ppm; IR: $\nu=3381,2962,2927,2835,1595,1558,1433,1237,1179$, $819,759 \mathrm{~cm}^{-1}$; HRMS (ES+) $\mathrm{m} / z$ calcd for $\mathrm{C}_{23} \mathrm{H}_{24} \mathrm{BN}_{2} \mathrm{O}[\mathrm{M}+\mathrm{H}]^{+}$ 355.1982, found 355.1989 .

\section{ASSOCIATED CONTENT}

\section{S Supporting Information}

The Supporting Information is available free of charge on the ACS Publications website at DOI: 10.1021/acs.joc.7b01331.

Crystallographic data for $3 a(\mathrm{CIF})$

Crystallographic data for 5a (CIF)

Included are copies of ${ }^{1} \mathrm{H},{ }^{13} \mathrm{C},{ }^{11} \mathrm{~B}$, and ${ }^{19} \mathrm{~F}$ NMR spectra for all compounds along with additional HTE screening data, computational characterization data and 2-D NMR and $\mathrm{X}$-ray crystallographic validation of the regioselective borylation (PDF)

\section{AUTHOR INFORMATION}

\section{Corresponding Author}

*E-mail: gmolander@sas.upenn.edu

ORCID

Geraint H. M. Davies: 0000-0002-5986-0756

Borna Saeednia: 0000-0003-2756-7827

Gary A. Molander: 0000-0002-9114-5584

\section{Author Contributions}

"These authors contributed equally

\section{Notes}

The authors declare no competing financial interest.

\section{ACKNOWLEDGMENTS}

This research was supported by the NIH/NIGMS (R01 GM111465). Frontier Scientific is acknowledged for their generous donation of potassium organotrifluoroborates. We thank University of Pennsylvania research facilities staff Dr. George Furst and Dr. Jun Gu for their help in the NMR elucidations, Dr. Pat Carroll and Dr. Brian Manor for X-ray crystallography support, and Dr. Simon Berritt for his expertise in highthroughput design. Dr. Christopher B. Kelly (UPenn) is acknowledged for his support, discussions, and guidance.

\section{REFERENCES}

(1) For reviews on bioisosterism: (a) Langmuir, I. J. Am. Chem. Soc. 1919, 41, 1543. (b) Patani, G. A.; LaVoie, E. J. Chem. Rev. 1996, 96, 3147. (c) Meanwell, N. A. J. Med. Chem. 2011, 54, 2529. (d) Francotte, P.; Goffin, E.; Fraikin, P.; Graindorge, E.; Lestage, P.; Danober, L.; Challal, S.; Rogez, N.; Nosjean, O.; Caignard, D. H.; Pirotte, B.; de
Tullio, P. J. Med. Chem. 2013, 56, 7838. (e) Pirotte, B.; de Tullio, P.; Florence, X.; Goffin, E.; Somers, F.; Boverie, S.; Lebrun, P. J. Med. Chem. 2013, 56, 3247.

(2) (a) Morgan, M. M.; Piers, W. E. Dalton Trans. 2016, 45, 5920. (b) Bosdet, M. J. D.; Piers, W. E. Can. J. Chem. 2009, 87, 8. (c) Abbey, E. R.; Liu, S.-Y. Org. Biomol. Chem. 2013, 11, 2060. (d) Campbell, P. G.; Marwitz, A. J. V.; Liu, S.-Y. Angew. Chem., Int. Ed. 2012, 51, 6074. (e) Noda, H.; Furutachi, M.; Asada, Y.; Shibasaki, M.; Kumagai, N. Nat. Chem. 2017, 9, 571.

(3) For 2,1-borazaronaphthalene synthetic methods: (a) Wisniewski, S. R.; Guenther, C. L.; Argintaru, O. A.; Molander, G. A. J. Org. Chem. 2014, 79, 365. (b) Molander, G. A.; Wisniewski, S. R. J. Org. Chem. 2014, 79, 6663. (c) Molander, G. A.; Wisniewski, S. R.; Amani, J. Org. Lett. 2014, 16, 5636. (d) Molander, G. A.; Amani, J.; Wisniewski, S. R. Org. Lett. 2014, 16, 6024. (e) Molander, G. A.; Wisniewski, S. R.; Traister, K. M. Org. Lett. 2014, 16, 3692. (f) Jouffroy, M.; Davies, G. H. M.; Molander, G. A. Org. Lett. 2016, 18, 1606. (g) Zhuang, F.-D.; Han, J.-M.; Tang, S.; Yang, J.-H.; Chen, Q.-R.; Wang, J.-Y.; Pei, J. Organometallics 2016, ASAP, 10.1021/acs.organomet.6b00811.

(4) (a) Rombouts, F. J. R.; Tovar, F.; Austin, N.; Tresadern, G.; Trabanco, A. A. J. Med. Chem. 2015, 58, 9287. (b) Vlasceanu, A.; Jessing, M.; Kilburn, J. P. Bioorg. Med. Chem. 2015, 23, 4453.

(5) (a) Scott, H. K.; Aggarwall, V. K. Chem. - Eur. J. 2011, 17, 13124. (b) Molander, G. A. J. Org. Chem. 2015, 80, 7837.

(6) References and reviews of $\mathrm{C}-\mathrm{H}$ borylation strategies: (a) Mkhalid, I. A. I.; Barnard, J. H.; Marder, T. B.; Murphy, J. M.; Hartwig, J. F. Chem. Rev. 2010, 110, 890. (b) Hartwig, J. F. Acc. Chem. Res. 2012, 45, 864. (c) Ros, A.; Fernández, R.; Lassaletta, J. M. Chem. Soc. Rev. 2014, 43, 3229. (d) Amaike, K.; Loach, R. P.; Movassaghi, M. Org. Synth. 2015, 92, 373. (e) Shinokubo, H. Proc. Jpn. Acad., Ser. B 2014, 90, 1. (f) Preshlock, S. M.; Ghaffari, B.; Maligres, P. E.; Krska, S. W.; Maleczka, R. E., Jr.; Smith, M. R., III J. Am. Chem. Soc. 2013, 135, 7572. (g) Saito, Y.; Segawa, Y.; Itami, K. J. Am. Chem. Soc. 2015, 137, 5193.

(7) Baggett, A. W.; Vasiliu, M.; Li, B.; Dixon, D. A.; Liu, S.-Y. J. Am. Chem. Soc. 2015, 137, 5536.

(8) Coventry, D. N.; Batsanov, A. S.; Goeta, A. E.; Howard, J. A. K.; Marder, T. B.; Perutz, R. N. Chem. Commun. 2005, 2172.

(9) Konishi, S.; Kawamorita, S.; Iwai, T.; Steel, P. G.; Marder, T. B.; Sawamura, M. Chem. - Asian J. 2014, 9, 434.

(10) Tajuddin, H.; Harrisson, P.; Bitterlich, B.; Collings, J. C.; Sim, N.; Batsanov, A. S.; Cheung, M. S.; Kawamorita, S.; Maxwell, A. C.; Shukla, L.; Morris, J.; Lin, Z.; Marder, T. B.; Steel, P. G. Chem. Sci. 2012, 3, 3505.

(11) Robbins, D. W.; Boebel, T. A.; Hartwig, J. F. J. Am. Chem. Soc. 2010, 132, 4068 .

(12) Paul, S.; Chotana, G. A.; Holmes, D.; Reichle, R. C.; Maleczka, R. E., Jr.; Smith, M. R., III J. Am. Chem. Soc. 2006, 128, 15552.

(13) Bocchi, V.; Palla, G. Synthesis 1982, 1982, 1096.

(14) (a) Becke, A. D. J. Chem. Phys. 1993, 98, 5648. (b) Lee, C.; Yang, W.; Parr, R. G. Phys. Rev. B: Condens. Matter Mater. Phys. 1988, 37, 785. (c) Vosko, S. H.; Wilk, L.; Nusair, M. Can. J. Phys. 1980, 58, 1200. (d) Stephens, P. J.; Devlin, F. J.; Chabalowski, C. F.; Frisch, M. J. J. Phys. Chem. 1994, 98, 11623. (e) Ditchfield, R.; Hehre, W. J.; Pople, J. A. J. Chem. Phys. 1971, 54, 724. (f) Hehre, W. J.; Ditchfield, R.; Pople, J. A. J. Chem. Phys. 1972, 56, 2257. (g) Hariharan, P. C.; Pople, J. A. Theor. Chim. Acta 1973, 28, 213.

(15) Frisch, M. J.; Trucks, G. W.; Schlegel, H. B.; Scuseria, G. E.; Robb, M. A.; Cheeseman, J. R.; Scalmani, G.; Barone, V.; Mennucci, B.; Petersson, G. A.; Nakatsuji, H.; Caricato, M.; Li, X.; Hratchian, H. P.; Izmaylov, A. F.; Bloino, J.; Zheng, G.; Sonnenberg, J. L.; Hada, M.; Ehara, M.; Toyota, K.; Fukuda, R.; Hasegawa, J.; Ishida, M.; Nakajima, T.; Honda, Y.; Kitao, O.; Nakai, H.; Vreven, T.; Montgomery, J. A., Jr.; Peralta, J. E.; Ogliaro, F.; Bearpark, M.; Heyd, J. J.; Brothers, E.; Kudin, K. N.; Staroverov, V. N.; Kobayashi, R.; Normand, J.; Raghavachari, K.; Rendell, A.; Burant, J. C.; Iyengar, S. S.; Tomasi, J.; Cossi, M.; Rega, N.; Millam, J. M.; Klene, M.; Knox, J. E.; Cross, J. B.; Bakken, V.; Adamo, C.; Jaramillo, J.; Gomperts, R.; Stratmann, R. E.; Yazyev, O.; Austin, A. J.; Cammi, R.; Pomelli, C.; Ochterski, J. W.; Martin, R. L.; 
Morokuma, K.; Zakrzewski, V. G.; Voth, G. A.; Salvador, P.; Dannenberg, J. J.; Dapprich, S.; Daniels, A. D.; Farkas, Ö.; Foresman, J. B.; Ortiz, J. V.; Cioslowski, J.; Fox, D. J. Gaussian 09, revision D.01; Gaussian, Inc.: Wallingford, CT, 2009.

(16) Schmidt, J. R.; Polik, W. F. WebMO Enterprise, version 14.0.004e; WebMO LLC: Holland, MI, 2013; http://www.webmo.net. (17) (a) Preshlock, S. M.; Plattner, D. L.; Malegres, P. E.; Krska, S. W.; Maleczka, R. E., Jr.; Smith, M. R., III Angew. Chem., Int. Ed. 2013, 52, 12915. (b) Roosen, P. C.; Kallepalli, B. A.; Chattopadhyay, B.; Singleton, D. A.; Maleczka, R. E., Jr.; Smith, M. R., III J. Am. Chem. Soc. 2012, 134, 11350.

(18) Vanchura, B. A.; Preshlock, S. M.; Roosen, P. C.; Kallepalli, V. A.; Staples, R. J.; Maleczka, R. E., Jr.; Singleton, D. A.; Smith, M. R., III. Chem. Commun. 2010, 46, 7724.

(19) Larsen, M. A.; Hartwig, J. F. J. Am. Chem. Soc. 2014, 136, 4287.

(20) Ishiyama, T.; Takagi, J.; Yonekawa, Y.; Hartwig, J. F.; Miyaura, N. Adv. Synth. Catal. 2003, 345, 1103.

(21) Vara, B. A.; Jouffroy, M.; Molander, G. A. Chem. Sci. 2017, 8, 530.

(22) Crudden, C. M.; Ziebenhaus, C.; Rygus, J. P. G.; Ghozati, K.; Unsworth, P. J.; Nambo, M.; Voth, S.; Hutchinson, M.; Laberge, V. S.; Maekawa, Y.; Imao, D. Nat. Commun. 2016, 7, 11065.

(23) (a) Balasubramaniyan, V. Chem. Rev. 1966, 66, 567. (b) Chu, J.H.; Tsai, S.-L.; Wu, M.-J. Synthesis 2009, 2009, 3757. 